NBER WORKING PAPER SERIES

\title{
PROBABILISTIC STATES VERSUS MULTIPLE CERTAINTIES: THE OBSTACLE OF UNCERTAINTY IN CONTINGENT REASONING
}

\author{
Alejandro Martínez-Marquina \\ Muriel Niederle \\ Emanuel Vespa \\ Working Paper 24030 \\ http://www.nber.org/papers/w24030 \\ NATIONAL BUREAU OF ECONOMIC RESEARCH \\ 1050 Massachusetts Avenue \\ Cambridge, MA 02138 \\ November 2017
}

The views expressed herein are those of the authors and do not necessarily reflect the views of the National Bureau of Economic Research.

NBER working papers are circulated for discussion and comment purposes. They have not been peer-reviewed or been subject to the review by the NBER Board of Directors that accompanies official NBER publications.

(C) 2017 by Alejandro Martínez-Marquina, Muriel Niederle, and Emanuel Vespa. All rights reserved. Short sections of text, not to exceed two paragraphs, may be quoted without explicit permission provided that full credit, including $\odot$ notice, is given to the source. 
Probabilistic States versus Multiple Certainties: The Obstacle of Uncertainty in Contingent Reasoning Alejandro Martínez-Marquina, Muriel Niederle, and Emanuel Vespa

NBER Working Paper No. 24030

November 2017

JEL No. C90,D81

\title{
ABSTRACT
}

We propose a new hypothesis, the Power of Certainty, to help explain agents' difficulties in making choices when there are multiple possible payoff-relevant states. In the probabilistic 'Acquiring-a-Company' problem an agent submits a price to a firm before knowing whether the firm is of low or high value. We construct a deterministic problem with a low and high value firm, where the agent submits a price that is sent to each firm separately. Subjects are much more likely to use dominant strategies in deterministic than in probabilistic problems, even though computations for profit maximization are identical for risk-neutral agents.

\author{
Alejandro Martínez-Marquina \\ Emanuel Vespa \\ Stanford University \\ 2127 North Hall \\ amm15@stanford.edu \\ University of California \\ Muriel Niederle \\ Santa Barbara, CA 93106 \\ Department of Economics \\ vespa@ucsb.edu \\ 579 Serra Mall \\ Stanford University \\ Stanford, CA 94305-6072 \\ and NBER \\ niederle@stanford.edu
}

An online appendix and instructions appendix are available at http://www.nber.org/data-appendix/w24030 


\title{
Probabilistic States versus Multiple Certainties: The Obstacle of Uncertainty in Contingent Reasoning
}

November 9, 2017

\author{
Alejandro Martínez-Marquina Muriel Niederle \\ (Stanford University) (Stanford University, NBER and SIEPR) \\ Emanuel Vespa \\ (UC Santa Barbara)
}

\begin{abstract}
We propose a new hypothesis, the Power of Certainty, to help explain agents' difficulties in making choices when there are multiple possible payoff-relevant states. In the probabilistic 'Acquiring-a-Company' problem an agent submits a price to a firm before knowing whether the firm is of low or high value. We construct a deterministic problem with a low and high value firm, where the agent submits a price that is sent to each firm separately. Subjects are much more likely to use dominant strategies in deterministic than in probabilistic problems, even though computations for profit maximization are identical for risk-neutral agents.
\end{abstract}

\section{Introduction}

We have to engage almost daily in hypothetical or conditional reasoning: making choices in environments with multiple possible payoff-relevant states. However, individuals routinely fail to make profit-maximizing choices in many such environments. ${ }^{1}$ A few papers have explicitly shown that even agents who are able to make profit-maximizing choices for a given state fail to do so once the state is uncertain (Esponda and Vespa, 2014 and Fragiadakis, Knoepfle and Niederle, 2017). In this paper we propose a new hypothesis that accounts for a large portion of this problem in simple settings. We claim that it is not (only) the multiple states that generate difficulties, but the fact that the states are uncertain.

\footnotetext{
${ }^{1}$ For evidence see for example Shafir and Tversky (1992), Charness and Levin (2009), Eyster and Weizsäcker (2010), Esponda and Vespa (2014), Enke (2017), Ngangoué and Weizsäcker (2017), Esponda and Vespa (2017a), Esponda and Vespa (2017b) and Araujo, Wang and Wilson (2017).
} 
This paper is part of a larger literature showing that, and trying to understand why, agents fail to make profit-maximizing choices. Results in strategic settings, even in environments where fairness concerns play no role, generated a literature accounting for agents' mistakes. ${ }^{2}$ There is also a literature in decision making that has documented behavioral biases that can explain failures to maximize payoffs. ${ }^{3}$ In addition, active work on heuristics (Tversky and Kahneman, 1974) often studies difficulties related to prediction or updating exercises. ${ }^{4}$ Here we focus on a simple decision problem with a few states in which the probability of each state is known and where evaluating the payoffs for an action only requires aggregating state-specific payoffs. We propose that aggregating over multiple contingencies is especially difficult when those contingencies (states) are uncertain. While there are several recent models aiming to understand difficulties in such basic environments (for example Sims, 2003, Gennaioli and Shleifer, 2010, Bordalo, Gennaioli and Shleifer, 2012, Köszegi and Szeidl, 2012, Gabaix, 2014, Caplin and Dean, 2015 and Caplin, Dean and Leahy, 2016), none directly differentiates between multiple probabilistic states and multiple certainties.

As an illustration, consider a two-state version of the Acquiring-a-Company problem (Bazerman and Samuelson, 1983), which we refer to as the 'probabilistic' problem. An agent decides whether or not to purchase a firm of value $v$. Once purchased, the value of the firm is $1.5 \times v$ and the agent knows that with equal chance the firm's value is either $v_{L}$ or $v_{H}$, where $0<v_{L}<v_{H}$. Without knowing the realization of $v$, the agent submits a price $p$ for the firm. The agent acquires the firm when $p \geq v$ and her payoff is $1.5 \times v-p$, otherwise the individual does not buy the firm and has a payoff of zero. Charness and Levin (2009) show that even in such a simple two-state version of the problem a large proportion of participants fail to select the profit-maximizing price. This could be because individuals have a hard time thinking about more than one state at the time or because, while agents are able to think about two states, they have a limited computational capacity to aggregate over states. These explanations can be summarized as the computational complexity hypothesis.

We propose a new hypothesis, namely that a prominent source of difficulties in optimization lies not only in there being two states, but also in the fact that which state materializes is unknown. That is, agents may be better at coping with two states or contingencies, as long as there is no uncertainty. We label this hypothesis as the Power of Certainty. Specifically, compare the 'probabilistic' problem described above to the following 'deterministic' problem in which we retain the complexity of two firms but eliminate any uncertainty. There are two firms of value known to the agent: one of value $v_{L}$ and another of value $v_{H}$. The agent submits a price $p$ that is sent to each firm separately and determines which firms she buys: if $p<v_{L}$, the agent buys none of the firms, if $v_{L} \leq p<v_{H}$, the

\footnotetext{
${ }^{2}$ For empirical evidence see for example Kagel and Levin (1986), Nagel (1995), Costa-Gomes and Crawford (2006), Costa-Gomes and Weizsäcker (2008), Ivanov, Levin and Niederle (2010), Eyster, Rabin and Weizsäcker (2015), Esponda and Vespa (2017b) and Fragiadakis, Knoepfle and Niederle (2017). For theoretical literature see e.g. Eyster and Rabin (2005), Jehiel (2005), Crawford and Iriberri (2007), Esponda (2008), Crawford, Costa-Gomes and Iriberri (2013).

${ }^{3}$ Failure for profit maximization can be due to various behavioral biases such a hyperbolic discounting (see e.g. Laibson, 1997 and O'Donoghue and Rabin, 1999) or probability weighting (Kahneman and Tversky, 1979).

${ }^{4}$ For recent empirical work see for example Mobius et al. (2014), Levin, Peck and Ivanov (2016), Vespa and Wilson (2016), Ambuehl and Li (2017), Enke and Zimmermann (2017); and for models see e.g. Rabin and Schrag (1999), Caplin and Leahy (2001), Bénabou and Tirole (2002) and Brunnermeier and Parker (2005).
} 
agent only buys the firm of value $v_{L}$ at a price $p$, and if $p \geq v_{H}$, the agent buys both firms, each at a price $p$. In the deterministic problem there is only one state and no uncertainty. However, the problem still requires that the agent evaluates two values of the firm, two "certainties." To compute the payoff-maximizing price, the agent has to determine the profits of buying only the firm $v_{L}$ or both firms $v_{L}$ and $v_{H}$. The profit-maximizing computations are identical to the computations a risk-neutral agent would need to do to optimize in the probabilistic problem (for details see the next section). We show that individuals are more likely to submit profit-maximizing prices in this deterministic problem than in the related probabilistic problem, providing evidence for the Power of Certainty, or the obstacle of uncertainty.

Specifically, we conduct an experiment using the probabilistic and deterministic problems described above. We consider the classic case where the dominant strategy of a risk-neutral or risk-averse agent is to submit a price $p=v_{L}$ (we use values such that $2 v_{L}<v_{H}$ ) and compare subjects' choices across the two treatments, the probabilistic treatment with probabilistic problems and the deterministic treatment with related deterministic problems. We show that substantially more subjects submit payoff-maximizing prices in the deterministic than in the probabilistic problems. However, one worry is that this result may not be due to higher instances of payoff maximization but rather due to rules of thumb that prescribe prices independent of the values of the firms and that may guide choices differently between the two treatments. We are particularly concerned with classifying too many subjects as payoff maximizers in the deterministic treatment, thereby overstating the role of our new hypothesis: the Power of Certainty. We therefore also study environments where we select the values of the two firms such that submitting $p=v_{H}$ is a dominant strategy (by guaranteeing that $\left.1.5 v_{L}>v_{H}\right)$. When we consider only subjects who both submit $p=v_{L}$ when $2 v_{L}<v_{H}$ and $p=v_{H}$ when $1.5 v_{L}>v_{H}$ as payoff maximizing, we find that there are twice as many payoff-maximizing subjects in the deterministic than in the probabilistic treatment.

One caveat of our analysis so far is that agents who are very risk seeking might prefer to submit a price $p=v_{H}$ even in the case where $2 v_{L}<v_{H}$ in the probabilistic treatment (though such a price $p=v_{H}$ is dominated in the deterministic treatment). While experimental subjects are in general risk averse, and often even excessively so given the low stakes, see Rabin (2000), we may still have some subjects who are risk seeking and hence we may have underestimated the fraction of subjects who are profit maximizers in the probabilistic treatment. We therefore introduce a measure of whether subjects are risk seeking in environments that mirrors the outcomes of the probabilistic treatment. Specifically, in three questions subjects choose between two lotteries that are constructed the following way: Take a specific $v_{L}$ and $v_{H}$ realization that is close to parameters encountered by subjects in the acquiring a company problem with $2 v_{L}<v_{H}$. Then the safer lottery corresponds to the lottery obtained when submitting a price $p=v_{L}$ in the probabilistic treatment, and the riskier lottery to the outcomes obtained when submitting a price $p=v_{H}$. This allows us to measure whether subjects might be risk seeking in the relevant domain. In addition, we can use those lottery choices to estimate the welfare loss subjects incur by having to submit prices in the probabilistic problems rather than directly selecting between the lotteries that correspond to the only two candidates of 
potentially payoff-maximizing prices: $v_{L}$ and $v_{H}$.

We show not only the existence, but also evaluate the quantitative significance of our proposed Power of Certainty hypothesis. To benchmark the extent to which agents are profit maximizing in a simple version of the problem, with only one firm of known value, we have a treatment where subjects make decisions in exactly such an environment. We use this treatment as well as the insight from lottery choices to quantify the effect of the Power of Certainty. We show that part of the welfare loss is due to moving from an environment that has one firm of known value to an environment with two firms of known value. We attribute this loss to what could be labelled as computational complexity. However, a substantial part of the welfare loss subjects incur when moving from a simple problem with one firm of known value to a problem where the firm might have two values is due to uncertainty, confirming the quantitative importance of the Power of Certainty in this environment.

Finally, we aim to shed some light as to the reason behind the Power of Certainty, why the problem with two firms of known value is so much simpler than the problem of one firm with two possible values. Clearly these two scenarios are distinct theoretically: one involves one state of the world that contains two firms, one of value $v_{L}$ and one of value $v_{H}$, and the other involves two states of the world, where each state corresponds to a given value of the firm, either $v_{L}$ or $v_{H}$. However, to compute payoff-maximizing prices, subjects have to take into account four outcomes: for each firm $v_{L}$ and $v_{H}$ the payoff from submitting either $p=v_{L}$ or $p=v_{H}$. Note that these four payoffs are exactly the ones that correspond to the outcomes of the lotteries corresponding to submitting $p=v_{L}$ or $p=v_{H}$.

To gain some insight into the subjects' thought processes, we had subjects provide incentivized advice to a new individual in the case of $v_{L}=20$ and $v_{H}=120$. Analysis of the advice suggests that in the deterministic problems subjects are much more likely to think of all four outcomes corresponding to $v, p \in\left\{v_{L}, v_{H}\right\}$. Furthermore, controlling for whether subjects' advice mentions those four outcomes accounts for the differences between the probabilistic and the deterministic treatments.

In a final treatment, subjects who first encounter the 'one firm of known value' version of the problem are then presented with either probabilistic or deterministic problems. We confirm that the Power of Certainty plays a role even among subjects who first made decisions in a simpler problem and hence may be more likely to think about the consequences of submitting a price $p$.

In the next section we describe the experimental design as well as some hypotheses and predictions. We also connect the Power of Certainty hypothesis to the psychology literature. The main results are in section 3. In section 4 we provide some insight as to the underlying cause of the Power of Certainty. We then discuss the related literature and possible applications, with specific attention to Li (2017), and finally, we summarize and conclude. 


\section{Experimental Design}

\section{$2.1 \quad$ Basic Environment}

The experimental design is built around a simplification of the Acquiring-A-Company game (Bazerman and Samuelson, 1983). A firm of value $v$ is for sale. The value of the firm is unknown to the agent, who knows that $v$ is equally likely to be $v_{L}$ or $v_{H}$, where $0<v_{L}<v_{H}<\infty$. The agent buys the firm if the agent's price $p$ is at least as high as $v$. If the agent does not buy the firm, her profit is zero. If she buys the firm, its value increases by 50 percent, so that profits equal $(1.5 v-p)$. Whenever subjects submit prices for firms, $p$ had to satisfy $p \in\{0,1,2, \ldots, 150\}$ and this was known to subjects.

When deciding about a price, note that any price $p$ not in $\left\{v_{L}, v_{H}\right\}$ is dominated. For instance, making an offer higher than $v_{H}$ is dominated by $p=v_{H}$, which also leads to buying the firm regardless of $v$ but at a lower price. Likewise, making an offer $p=v_{L}$ dominates any price strictly between $v_{L}$ and $v_{H}$. Finally, offering a price below $v_{L}$ guarantees that a firm will not be purchased, but is dominated by $p=v_{L}$, which leads to either zero or strictly positive profits.

Consider the case of a risk-neutral agent. Depending on the agent's price $p$, the expected profit is:

$$
\pi(p)=\left\{\begin{array}{ll}
\frac{1}{2}\left(1.5 v_{L}-v_{L}\right) & \text { if } p=v_{L} \\
\frac{1}{2}\left(1.5 v_{H}-v_{H}\right)+\frac{1}{2}\left(1.5 v_{L}-v_{H}\right) & \text { if } p=v_{H}
\end{array} .\right.
$$

Note that when $1.5 v_{L}<v_{H}$, the agent receives a negative profit when $v=v_{L}$ and $p=v_{H}$. She may still prefer to submit $p=v_{H}$ if the gains in case of $v=v_{H}$ are sufficiently large. A risk-neutral agent is indifferent between a price of $v_{H}$ or $v_{L}$ when $2 v_{L}=v_{H}$. When $2 v_{L}$ is strictly lower than $v_{H}$, both a risk-neutral and a risk-averse agent prefer $p=v_{L}$. A risk-seeking agent may still prefer to submit a price $p=v_{H}$ due to the positive returns when $v=v_{H}$. In contrast, when $1.5 v_{L} \geq v_{H}$, all agents prefer a price $p=v_{H}$ to a price $p=v_{L}$.

\subsection{Main Treatments}

We first describe the two main treatments of our between-subjects design, the Probabilistic (PROB) and the Deterministic (DET) treatment. In both treatments subjects faced the same five parts, differing only as much as described below.

\section{Probabilistic Treatment (РROB)}

In PROB the subject submits a price for a single firm that could have either of two values in $\left\{v_{L}, v_{H}\right\}$, each with 50 percent chance. The subject did not know the value of the firm when submitting a 
price. If $p<v_{L}$, the subject buys no firm and the profit is zero. If $v_{L} \leq p<v_{H}$, she only buys the firm if the firm is of value $v_{L}$ at a price $p$. Finally, if $p \geq v_{H}$, she always buys the firm.

In order to simplify the comparison with DET described next, payoffs are multiplied by two, so that expected profits are:

$$
\pi^{\text {Prob }}(p)= \begin{cases}0 & \text { if } p<v_{L} \\ \left(1.5 v_{L}-p\right) & \text { if } v_{L} \leq p<v_{H} \\ \left(1.5 v_{H}-p\right)+\left(1.5 v_{L}-p\right) & \text { if } v_{H} \leq p\end{cases}
$$

\section{Deterministic Treatment (DET)}

In DET there are two firms for sale, one of value $v_{L}$ and another of value $v_{H}$. The subject can submit a unique price $p$ that is sent to each firm separately. The price $p$ determines whether the agent buys none, one or two firms, each at price $p$. Profits are given by:

$$
\pi^{\text {Det }}(p)= \begin{cases}0 & \text { if } p<v_{L} \\ \left(1.5 v_{L}-p\right) & \text { if } v_{L} \leq p<v_{H} \\ \left(1.5 v_{H}-p\right)+\left(1.5 v_{L}-p\right) & \text { if } v_{H} \leq p\end{cases}
$$

\section{Parts 1-5 of the experiment}

In part 1 of each treatment subjects faced 20 rounds with values $\left\{v_{L}, v_{H}\right\}$ such that $2 v_{L}<v_{H}$. The first question always had $v_{L}=20$ and $v_{H}=120$. For the other 19 questions we chose the two values $\left\{v_{L}, v_{H}\right\}$ randomly under the following constraints: Both $v_{L}$ and $v_{H}$ had to be even numbers and furthermore $10 \leq v_{L} \leq 30$ and $80 \leq v_{H} \leq 140 .{ }^{5}$ We decided to select those values randomly in order to not bias our results by unknowingly selecting values (or sequences of values) that would favor specific results (for a discussion on the value of random games see also Fragiadakis, Knoepfle and Niederle, 2017). While all subjects saw the same 19 sets of values $\left\{v_{L}, v_{H}\right\}$, the order was randomized at the subject level. Because subjects could submit any price $p \in[0,150] \cap \mathbb{N}$, underbidding is always possible and overbidding (which can lead to losses) is bounded. Since in part 1 all values $\left\{v_{L}, v_{H}\right\}$ satisfied $2 v_{L}<v_{H}$, a risk-neutral or risk-averse subject in PROB would optimally submit a price of $p=v_{L}$ in all rounds. In DET, submitting a price of $p=v_{L}$ is the dominant strategy.

Part 2 consisted of 5 rounds and values $\left\{v_{L}, v_{H}\right\}$ are selected such that $1.5 v_{L}>v_{H} .{ }^{6}$ This means it is optimal to submit $p=v_{H}$ regardless of risk attitudes, in both PROB and DET. We chose

\footnotetext{
${ }^{5} \mathrm{We}$ constrained the selected values to be even numbers so that computations such as $1.5 v$ result in integers. Subjects are not told the domains from which the values are drawn. They are simply shown the realizations for a specific round and asked to submit a price. The 19 pairs we implemented in rounds $2-20$ were: $\{10,86\},\{10,96\}$, $\{12,112\},\{12,136\},\{12,138\},\{14,122\},\{14,140\},\{16,98\},\{16,106\},\{18,80\},\{18,86\},\{18,90\},\{18,122\}$, $\{20,130\},\{22,106\},\{24,126\},\{24,128\},\{24,134\},\{28,126\}$.

${ }^{6}$ In the experiment there was a clear "break" between part 1 and part 2, see the Instructions Appendix for details. Given that the problems require computations, we were concerned that mixing up problems where (for non risk-seeking
} 
random combinations of values with the constraint that both $v_{L}$ and $v_{H}$ had to be even numbers and furthermore $48 \leq v_{L} \leq 54,54 \leq v_{H} \leq 64$ and $v_{L} \neq v_{H} \cdot{ }^{7}$ While once more all subjects saw the same set of five values $\left\{v_{L}, v_{H}\right\}$, the order was randomized at the subject level.

In part 3 subjects provided incentivized advice for the case $v_{L}=20$ and $v_{H}=120$. They recommended what price to submit and why to a future participant, which we refer to as the advisee. We told them that the advisee will be presented with advice from 5 different participants and that she will select which of the 5 pieces of advice was the most helpful. We told participants that they would receive the profits the advisee made in this problem provided that their advice is the one selected by the advisee. By forcing $v_{L}=20$ and $v_{H}=120$ to be in the first round of part 1 , we ensure that all subjects have the same amount of experience (none) when they encountered this problem and 24 rounds passed before they provided advice.

In part 4, subjects are presented with 10 rounds that are similar to those in part 1 . However, now, subjects faced the treatment of the problem they did not face so far. This means that subjects who faced 20 rounds of probabilistic problems in part 1 faced 10 rounds of deterministic problems in part 4 and vice versa. In round 1 of part 4 we fix $v_{L}=20$ and $v_{H}=120$. For the remaining 9 rounds we pre-selected 9 pairs with the same criterion as described in part 1, though all were different from those in part 1.8 Different subjects faced these pairs in a different random order.

Part 5 consists of three questions in which subjects selected one of two lotteries. In all lotteries there was a 50-50 chance of obtaining a low $\left(\pi^{L}\right)$ or a high $\left(\pi^{H}\right)$ payoff. For simplicity we describe a lottery as $\mathcal{L}\left(\pi^{L}, \pi^{H}\right)$. In the three questions agents chose between $\mathcal{L}(0,10)$ and $\mathcal{L}(-250,140)$, $\mathcal{L}(0,20)$ and $\mathcal{L}(-180,120)$, and $\mathcal{L}(0,30)$ and $\mathcal{L}(-70,80)$, respectively. These lotteries correspond to PROB cases $\left(v_{L}, v_{H}\right)=\{\{10,140\},\{20,120\},\{30,80\}\}$, where the agent either submits $p=v_{L}$ or $p=v_{H}$, respectively. ${ }^{9}$ All subjects faced these three questions in that same order, though they were not told how those lotteries were constructed.

Throughout part 1, and in fact throughout all parts of PROB and DET, and throughout all other treatments, subjects did not receive any feedback. That is, after each question they answered (e.g. submitted a price, in part 1), subjects simply received the next question. On the one hand, Charness and Levin (2009) document that feedback and experience does not remove overbidding $\left(p>v_{L}\right)$ in the probabilistic case in a problem akin to those of part 1 . In addition, while it may be interesting to address how learning changes the answers, we were mostly concerned that learning would be much more rapid in DET than in PROB. The reason is that feedback may be more informative in DET than in PROB. In PROB, feedback is the outcome of a lottery: Sometimes submitting a high price $p=v_{H}$, while leading to expected losses, may result in large gains. In DET, feedback consists of the profit.

agents) $p=v_{L}$ and $p=v_{H}$ was a dominant strategy would increase the fraction of subjects who would not submit payoff maximizing prices due to computation mistakes.

${ }^{7}$ The five pairs we implemented were: $\{48,54\},\{50,54\},\{52,58\},\{52,64\},\{54,58\}$.

${ }^{8}$ The 9 pairs we implemented in rounds $2-10$ of part 4 were: $\{10,82\},\{10,110\},\{14,98\},\{16,80\},\{24,122\}$, $\{26,96\},\{28,92\},\{28,96\},\{30,88\}$.

${ }^{9}$ The $\{10,140\}$ and $\{30,80\}$ combinations correspond to the most extreme values subjects could have encountered in part 1 and part 4 . 
In fact, in all our part 1 problems, $3 v_{L}<v_{H}$, which implies that submitting a price $p=v_{H}$ leads to a negative profit. Indeed, experiments have shown that learning may be slower when the feedback is the result of a lottery rather than the expected outcome, for a specific example with such a direct comparison see Bereby-Meyer and Roth (2006).

\subsection{Advisee Treatments}

We have a Probabilistic (ADVProB) and a Deterministic Advisee (ADVDET) treatment. The subject (advisee) goes through the same instructions and understanding tests as subjects in the main treatments. A subject then receives the first question of part 1 from the main treatment, where the values are $v_{L}=20$ and $v_{H}=120$. Before being given a chance to submit a price for that question, the subject receives five pieces of advice, one from each of five subjects from the corresponding main treatment. The subject sees one piece of advice at the time and answers whether the advice is useful (selecting from: very useful, somewhat useful or not useful at all). Subsequently, the subject sees all 5 pieces of advice at once and indicates which advice is most helpful. Finally, the subject submits a price for the $\{20,120\}$ question and then faces the same 19 questions of part 1 of the main treatment, in random order. The subject then faces part 2 of the main treatment.

\subsection{One-Firm Treatments}

In the One-Firm treatments subjects are first confronted with 25 rounds of a simplification of part 1 and part 2 of the main treatments. Specifically, in the first 25 rounds (part 1 and part 2) each subject submits a price for only a single firm of which they know the value. These 25 rounds correspond to part 1 and part 2 of the main treatments where, in each round, we randomize at the individual level which value (which firm) the subject can buy. In part 3, part 4 and part 5 subjects face part 1, part 2 and part 5 of either PROB or DET.

We implement two One-Firm treatments. In the Probabilistic One-Firm treatment, subjects are presented with the instructions that correspond to PROB. After they have read the instructions, but before they start part 1 we tell them that they will actually know the value of the firm before they submit a price. In the Deterministic One-Firm treatment, subjects read the instructions that correspond to DET. Once they finish reading those instructions we tell them that in part 1 there will actually be only one of the two firms available for sale and that they will know which one of the two they can buy. That is, in each treatment we reduce the two-contingency problem to a one-contingency problem, but retain the initial description of the two-contingency problem. Part 3 , part 4 and part 5 of the Probabilistic (Deterministic) One-Firm treatment correspond to part 1, part 2 and part 5 of PROB (DET). 


\subsection{Hypotheses and Predictions}

A common approach to showing the difficulties with conditional reasoning is to show that subjects are not making profit-maximizing choices, and investigating whether some changes to the problem reduce those difficulties, see Charness and Levin (2009), Enke and Zimmermann (2017). A few papers directly compare choices when subjects know the contingency (or as good as) to when they did not, such as comparing the performance in the the first two parts of the One-Firm treatment to part 1 of PROB, see for example, Esponda and Vespa (2014), Ngangoué and Weizsäcker (2017), Fragiadakis, Knoepfle and Niederle (2017). ${ }^{10}$ We expect to replicate that subjects are somewhat able to compute profit-maximizing prices when there is a single firm of known value in the first parts of the One-Firm treatment, but that there are fewer cases of profit-maximizing choices in PROB. We start by presenting the corresponding hypothesis, an early discussion of which can be found in Hamilton (1878), see also Lennie (2003) for a more recent discussion. ${ }^{11}$

\section{Hypothesis 0: Computational Complexity (Complexity) One reason why subjects may} find it more difficult to make payoff-maximizing choices in PROB compared to ONEFIRM is that the problem is harder when there are two firms or two possible contingencies to consider. This could be either because subjects have a hard time thinking about two values, or because they find it difficult to aggregate this information. This may include that the stakes are simply too small to warrant expending costly computations needed to achieve payoff maximization. We summarize these issues as computational complexities.

Computational complexity is one potential explanation why agents who can compute the best response in each contingency fail to do so when there are multiple contingencies. In this paper we

\footnotetext{
${ }^{10}$ Esponda and Vespa (2014) consists of an extreme version of such a result where, while there are multiple contingencies, the agent has a dominant action in one contingency and is indifferent between her actions in all other contingencies. They show that many subjects who understand what to do in each contingency still fail to behave optimally when there is uncertainty over which contingency is realized. Specifically, they have a voting experiment where there is a true state of the world, either red or blue. A committee consisting of 2 robots and a subject vote for red or blue. If the majority vote corresponds to the true state, the subject receives a strictly positive payoff, otherwise the subject receives zero. One example of a problem subjects face is that the two computers use the following rule, which is known to subjects: If the state of the world is red, computers are programmed to vote red; if the state of the world is blue, computers mix independently between voting red or blue, with each vote being equally likely. The subject does not know the state of the world and does not know the votes of the computers when submitting her own vote. Note that the vote of the subject is pivotal only when one computer votes red and the other blue, which only happens in the blue state, so, the subject has a dominant strategy to vote blue. Put differently, in all contingencies the vote of the subject is irrelevant, apart from in one case, in which the subject has a strict incentive to vote blue. Only about 19 percent of subjects eventually always submit the dominant strategy. In a sequential treatment where subjects are shown the computers' votes before voting themselves, approximately 70 percent of subjects eventually always vote blue.

${ }^{11}$ Hamilton (1878) poses it in the following way: "Supposing that the mind is not limited to the simultaneous consideration of a single object, a question arises, How many objects can it embrace at once? You will recollect that I formerly stated that the greater the number of objects among which the attention of the mind is distributed, the feebler and less distinct will it be its cognizance of each. "Pluribus intentus, minor est ad singula sensus."' A more recent presentation is in Lennie (2003), who argues that "The need for energy management provides an interesting physiological perspective on a traditional view of attention as adaptation to the brain's limited capacity to process information: energy limitations require that only a small fraction of the machinery can ever be engaged concurrently."
} 
introduce an additional explanation, namely that multiple contingencies are especially hard to handle in the case of uncertainty about the state of the world, rather than when there are simply multiple certainties. $^{12}$

Hypothesis 1: The Power of Certainty (PoC) In PROB we have one firm that is equally likely to have one of two possible values, while in DET we have two firms, one of each of the two potential values in PROB. In both PROB and DET subjects have to submit one price that applies to all possible values the firm can have. The two treatments differ, however, in one dimension: While in PROB subjects submit a price for one firm with two possible values $v_{L}$ and $v_{H}$, subjects in DET submit a price that is transmitted separately to the firm $v_{L}$ and the firm $v_{H}$. The expected profits in PROB are exactly the same as the realized profits in DET. That is, in both cases there are two possible values of the firm to consider. The main difference between the two treatments is that while in PROB there is uncertainty over the contingency (state) that is realized, in DET there is no uncertainty about which contingency is realized. DET shares the lack of uncertainty with ONEFIRM, while, however, keeping the complexity associated with two contingencies from PRoB.

The Power of Certainty ( $P o C$ ) hypothesis claims that individuals may have a difficulty to compute payoff-maximizing actions when there is uncertainty about which state is realized. When both contingencies of the firm exist, however, computing payoff-maximizing actions may be easier. While there is no active literature on this topic in economics, there are several concepts that hint towards the fact that it is difficult for individuals to think about uncertainty. In hindsight bias, see Fischhoff (1975), individuals underestimate the likelihood that other states could have occurred, basically having a hard time to think about outcomes being uncertain. In fact, there is substantial evidence that shows that young children tend to view the world in a deterministic manner and often attribute causal effects to situations of chance, a finding that does not disappear with age (for a survey see Langrall and Mooney, 2005). ${ }^{13}$ Believing in superstition or fate are some of the ways by which individuals reduce the uncertainty inherent in life. In a similar vain, the "control heuristic" (Thompson, Armstrong and Thomas, 1998) provides one possible rationale for the $\mathrm{PoC}$. This heuristic is a shortcut that involves two elements: the intention of the subject to achieve an outcome (here the payoff associated with $p=v_{H}$ and $v=v_{H}$ ) and the perceived connection between the subject's action and the desired outcome.

In psychology, Hoffrage et al. (2002) find that individuals are better at Bayesian updating when probabilistic problems are restated using frequencies. One interpretation of our DET treatment is that it presents a frequentist problem (50 percent of the existing firms are of value $v_{L}$ and 50 percent are of value $v_{H}$ ), and, hence, we too compare a frequentist problem in DET to a probabilistic problem in PROB. However, Hoffrage et al. (2002) use their results to dismiss Kahneman and Tversky (1972)

\footnotetext{
${ }^{12}$ Strictly speaking, we have only risk and not Knightian uncertainty in our experiment, but we will use the terms interchangeably.

${ }^{13}$ In fact, Fischbein and Schnarch (1997) suggest that probabilistic reasoning requires the construction and development of new intuitions.
} 
by arguing that heuristics and biases may simply be due to subject confusion when faced with an unfamiliar (probabilistic) frame. We, instead, propose that there is a fundamental difference between frequencies and probabilities, that there is a Power of Certainty. In our experiment PROB and DET are not different frames of the same problem, but are different problems.

To ascertain the role of $P o C$ in accounting for subjects' difficulties in PROB we compare behavior in parts 1 and 2 between PROB and DET. Specifically, if subjects find it easier to think through the problem in the absence of uncertainty, we would expect more subjects to select $p=v_{L}$ in part 1 of DET than in PROB. However, when we compare the occurrence of prices $p=v_{L}$ across part 1 of PROB and DET, we may have underestimated the number of profit maximizing subjects in PROB. Specifically, there is one reason why subjects in PROB might submit $v_{L}<p=v_{H}$ : the subject might be (very) risk seeking.

Hypothesis 2: Risk Seeking (Risk) While any price $p \neq v_{L}$ is strictly dominated in DET, this is the case in PROB only for subjects who are not (very) risk seeking. For subjects who are risk seeking, submitting a price $p=v_{H}$ can be profit maximizing. Subjects' lottery choices in part 5 provide a direct test whether they prefer the risky lottery that corresponds to $p=v_{H}$ over the safer lottery that corresponds to $p=v_{L}$. We can then correlate their lottery choices with their propensity to submit $p=v_{L}$ in part $1 .^{14}$

The lottery choices in part 5 provide a risk-seeking measure that closely mirrors the choices of submitting a price in part 1 (see Niederle, 2016 for a discussion of the virtues of such an approach). In addition, we can use the lottery choices to address losses due to presenting the complicated probabilistic problem rather than the simple lottery choices that result from submitting either $p=v_{L}$ or $p=v_{H}$ (see also Ambuehl, Bernheim and Lusardi, 2014).

Since we want to establish the importance of the $P o C$ hypothesis, it is not only important that we do not underestimate the number of profit maximizing subjects in PROB, but also that we do not overestimate the number of profit maximizing subjects in DET. Specifically, we do not want to misclassify subjects who use a rule of thumb as profit maximizers in DET. Therefore, we introduce part 2 of the main treatments where $p=v_{H}$ is the profit maximizing price (in both DET and PROB). Hence, subjects who submit $p=v_{L}$ in any deterministic (or probabilistic) problem of two firms independent of the actual values of $v_{L}$ and $v_{H}$ will reveal themselves as not profit maximizing in part 2.

Hypothesis 3: Rules of Thumb (Rules of Thumb) There are several rules of thumb that subjects may use that would make them appear to be profit maximizing in part 1. For example, subjects could submit $p=v_{L}$ in part 1 independent of the values of $v_{L}$ and $v_{H}$ since it feels "cheaper"

\footnotetext{
${ }^{14}$ While there are many measures addressing how risk averse an individual is, there is no standardized measure for how risk seeking an individual is. Recall that even a risk-neutral, let alone a risk-averse individual finds $p=v_{L}$ to be the dominant choice in part 1 of PROB.
} 
than $p=v_{H}$. Alternatively, it could be that subjects prefer to think about one firm only. While in PROB the subject can buy at most one firm, she can buy two firms in DET if she submits a price $p \geq v_{H}$. A subject in DET who wants to avoid buying two firms could submit a price below $v_{H}$. Such a "constraint" would vastly simplify the problem, and could lead to fewer prices that can lead to losses and more selections of $p=v_{L}$.

We need a simple way to test whether rules of thumb lead to a high occurrence of $p=v_{L}$ (independent of the values of $v_{L}$ and $v_{H}$ ) especially in DET. Therefore, in part 2 of the main treatments, we change the values $\left\{v_{L}, v_{H}\right\}$ such that $1.5 v_{L}>v_{H}$ and the dominant strategy is to submit a price $p=v_{H}$. We use the combination of submitted prices in part 1 and part 2 to better understand the behavior of subjects and assess whether they consistently use profit maximizing strategies and have an understanding of the problem.

Note that a rule of thumb that leads subjects to either always submit $p=v_{H}$ or mix between $\left\{v_{L}, v_{H}\right\}$ is easily recognized as dominated in DET, though in PROB such subjects may be misclassified as being very risk seeking albeit profit maximizing in part 1.

\subsection{Understanding the Power of Certainty}

We aim to not only show the significant role of the $P o C$ hypothesis, but also its relative importance. We first use the comparison between ONEFIRM and PROB to assess the quantitative problem that is in general associated with Complexity. We then use the relative outcome of DET between on the one hand ONEFIRM, which will account for the difference between a single known certainty and multiple known certainties, and PROB, which will account for the difference between two contingencies that are certain versus uncertain. Second, we will use the choices in part 5 that correspond to lotteries where the agent either submits $p=v_{L}$ or $p=v_{H}$ in probabilistic problems. We compare the performance of subjects who have to compute the four possible outcomes $v, p \in\left\{v_{L}, v_{H}\right\}$ themselves in probabilistic problems rather than receiving those four outcomes directly and only choosing between the lotteries that correspond to submitting $v_{L}$ or $v_{H}$, respectively.

To begin to investigate the underlying reasons for the $P_{O} C$, note that in both PROB and DET subjects have to consider all possible four outcomes $v, p \in\left\{v_{L}, v_{H}\right\}$ - just as we explicitly do for subjects choosing between lotteries in part 5 . We first use the (incentivized) advice subjects provide for a different participant to assess whether subjects, in at least some form, mention those four outcomes. We then assess to what extent this can account for differences between DET and PROB. Second, after providing advice, in part 4, subjects submitted prices in part 1-style problems, though now in the other treatment. That is, subjects in DET encountered probabilistic problems and vice versa. We assess whether mentioning all four outcomes has a significant impact on the performance of subjects in part 4. Third, we use the Advisee treatments ADVPROB and ADVDET for some external verification of the role of thinking about all four outcomes. Finally, we assess the role of forcing subjects to think about outcomes by having subjects first solve 25 rounds of problems involving a single firm with known value in ONEFIRM. This may increase the understanding of the problem and 
help subjects to focus on outcomes when submitting prices. We study whether after that experience, differences between probabilistic and deterministic problems are still large and present.

\subsection{Procedures}

Our subjects are Amazon Turk workers located in the US with a rating of 90 percent or higher. Subjects received a link to a qualtrics survey (see the Instructions Appendix which contains all the surveys). Participants knew that if they made more than two mistakes on the instructions for part 1 of any treatment, they were not allowed to continue the experiment. Upon finishing the experiment, we asked survey questions pertaining to the sex, age, ethnicity, education and state of residence of the participant. We have a total of 880 Amazon Turk workers with unique IP addresses, of which 44.8 percent are female, 74.8 percent are White, 49 percent are at or below the median age of 32 and 38.9 percent have low schooling ('Some college,' 'High School' or lower education level). ${ }^{15}$

Probabilistic and Deterministic Treatments We aimed to recruit 200 participants per treatment. In total 425 Amazon Turk workers with a unique IP address started the experiment (211 PROB and 213 in DET), but 54 made more than 2 mistakes in the instructions for part $1 .{ }^{16}$ Eventually, we were left with 188 and 183 subjects in PROB and DET, respectively.

Payments in PROB and DET were determined as follows. A participant received $\$ 4$ for finishing the survey, as well as another $\$ 4$ at the beginning of part 1 to which they can add or subtract depending on their choices in the experiment. In each of part 1, part 2 and part 4 we randomly select one round for payment. In part 3 subjects are paid the advisee's payoff in case their advice was selected. In part 5 we randomly select one of the three lottery choices and pay subjects based on their chosen lottery. Payoffs in all questions are expressed in points which are subsequently converted to dollars. In parts 1, 3 and 5 we paid 3 cents per point. In parts 2 and 4 we paid 1 cent per point. ${ }^{17}$ On average a participant received $\$ 8.7$ (including the $\$ 4$ for finishing the survey) and on average the survey took approximately 40 minutes to complete.

We paid special attention to ensure that the instructions of PROB and DET are as similar as possible. For example, when describing the problem in PROB we use the phrase "transaction of the company," while in DET we use "transaction for each company." In Appendix E we provide a brief summary of how we explained each problem in the instructions and show screenshots for part 1 round 1 of PROB and DET (see Figure 9). In the Instructions Appendix we provide the full instructions.

\footnotetext{
${ }^{15}$ The regression tables presented in the results section and the appendix control for these demographic variables. In particular, we construct a gender dummy, an ethnicity dummy that takes value 1 if the responder selected 'White' and 0 otherwise, an age dummy that takes value 1 if the responder is at or below the median age, and a low schooling dummy that takes value 1 if the responder selected 'Some college,' 'High School' or lower education level.

${ }^{16}$ Of the 54 subjects, 23 and 31 correspond to PROB and DET, respectively. The difference in the drop rate is not significant across treatments.

${ }^{17}$ Note that in part 2, the average payment in points from submitting the dominant price is higher than in part 1 , and furthermore, there are fewer problems in part 2. We therefore used a lower exchange rate in part 2. However, just to ensure subjects do not react too strongly to only 1 cent payments, we also used that payment in part 4 . We find no indication that the reduced payments have an effect.
} 
Advisee Treatments We recruited 90 Mturkers who had not participated in an earlier treatment, half of which were assigned to each treatment. We drop 4 subjects in the probabilistic and 5 subjects in the deterministic version who make more than two mistakes in the instructions. Eventually we are left with 41 subjects in ADVPROB and 40 in ADVDET. ${ }^{18}$

One-Firm Treatments We recruited 468 Mturkers (233 in the probabilistic and 235 to the deterministic version) who had not participated in an earlier treatment. However, 40 subjects made more than two mistakes in the instructions for part 1 which leaves us with 216 subjects in the Probabilistic and 212 in the Deterministic One-Firm treatment. ${ }^{19}$

\section{The Power of Certainty}

We first classify subjects based on their choices in part 2 where $p=v_{H}$ is a dominant strategy in both PROB and DET. The difference between these two treatments provides a first indication of the PoC hypothesis. We then turn to the classic form of the acquiring-a-company problem in part 1, which is perhaps more difficult and where $p=v_{L}$ is a dominant strategy in DET and in PROB for subjects who are not (very) risk seeking. We assess whether subjects are able to adjust their prices to the values of the two firms to address the role of Rules of Thumb. We then examine whether risk-seeking subjects account for prices $p=v_{H}$ in part 1 of PROB and hence the Risk hypothesis. Finally, we assess the relative importance of $P o C$ in the more general problem of Complexity. We present all our results using classification of subjects into types. This allows us to address both the role of rules of thumbs as well as of risk-seeking preferences. We show in Appendix A that our findings are robust to instead considering the distribution of prices. ${ }^{20}$

\footnotetext{
${ }^{18} \mathrm{~A}$ participant received $\$ 4$ for finishing the survey. Participants are then endowed with another $\$ 4$ at the beginning of part 1 and can add/subtract the payment from one randomly selected round for part 1 and one randomly selected round for part 2. Payoffs are expressed in points and transformed to dollars at 3 cents per point in part 1 and 1 cent per point in part 2. On average a participant received $\$ 8.2$.

${ }^{19}$ Of the 40 subjects, 17 and 23 correspond to the probabilistic and deterministic version, respectively. The difference in the drop rate is not significant across treatments. A participant received $\$ 4$ for finishing the survey. Participants are then endowed with another $\$ 4$ at the beginning of part 1 and can add/subtract the payment from one randomly selected round for parts 1, 2, 3 and 4, and from one randomly selected lottery in part 5 . In parts 1,3 and 5 we paid 3 cents per point. In parts 2 and 4 we paid 1 cent per point. On average a participant received $\$ 8.6$. While it is possible for subjects to have total earnings below the $\$ 4$ they were endowed with to potentially lose during the experiment, of the 880 subjects that are in our final sample, only 24 did so, for those subjects we paid only the $\$ 4$ they were guaranteed, and we did not implement their full losses above the $\$ 4$ they were endowed with initially.

${ }^{20}$ The approach we follow in the text, which classifies subjects into types, demands consistent behavior from subjects. In contrast, the analysis on submitted prices in Appendix A does not rely on consistency of behavior. This means that there will be a difference in levels. For example, the aggregate frequency of prices $p=v_{L}$ is higher than the frequency of subjects who submit $p=v_{L}$ in all periods. While there is a difference in levels, our conclusions comparing outcomes across treatments are not affected. In the appendix we also present classifications of subjects into types allowing for small deviations, and again, we reach the same conclusions.
} 


\subsection{Part 2 Behavior}

In part 2 (rounds 21-25) the values $\left\{v_{L}, v_{H}\right\}$ are such that $1.5 v_{L}>v_{H}$ which makes submitting the price $p=v_{H}$ a dominant strategy in both PROB and DET. Figure 1a shows the proportion of subjects who placed a price $p=v_{H}$ from round $n$ to 25 , for $21 \leq n \leq 24$. Subjects in DET are about 15 percentage points more likely to do so than subjects in PROB. Another price that may be focal, though dominated, is $p=v_{L}$. Figures $1 \mathrm{~b}$ and $1 \mathrm{c}$ show the fraction of subjects who consistently submit $p=v_{L}$ and who strictly mix between $p=v_{L}$ and $p=v_{H}$ from round $n$ onwards, for $21 \leq n \leq 24$, respectively. The use of non-optimal focal prices is somewhat more prevalent in PROB than in DET. Finally, we show the fraction of subjects who consistently submit a dominated price that is non-focal, that is $p \notin\left\{v_{L}, v_{H}\right\}$. Once more this strategy is slightly more prevalent in PROB than in DET.

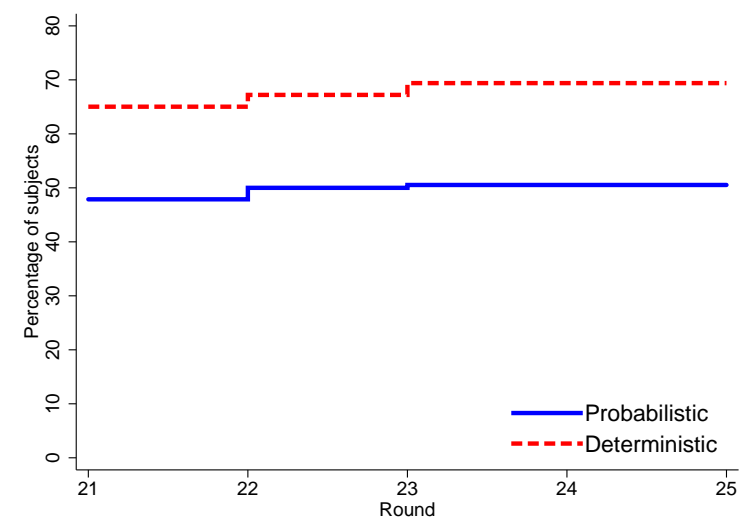

(a) $p=v_{H}$.

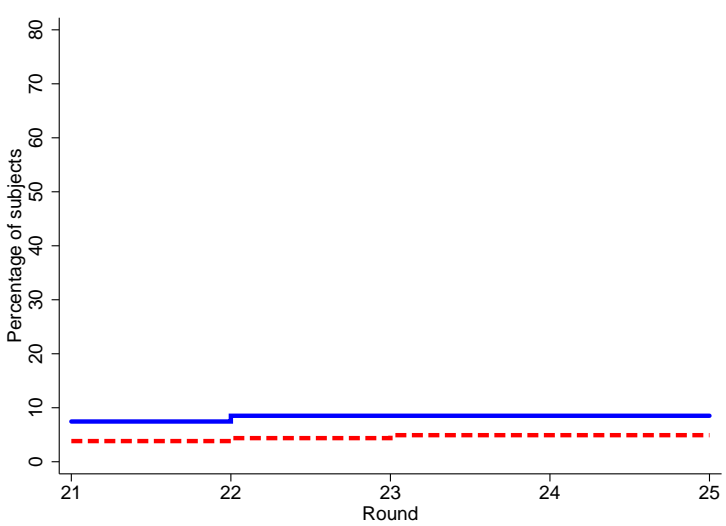

(c) $p \in\left\{v_{L}, v_{H}\right\}$ and at least one of each .

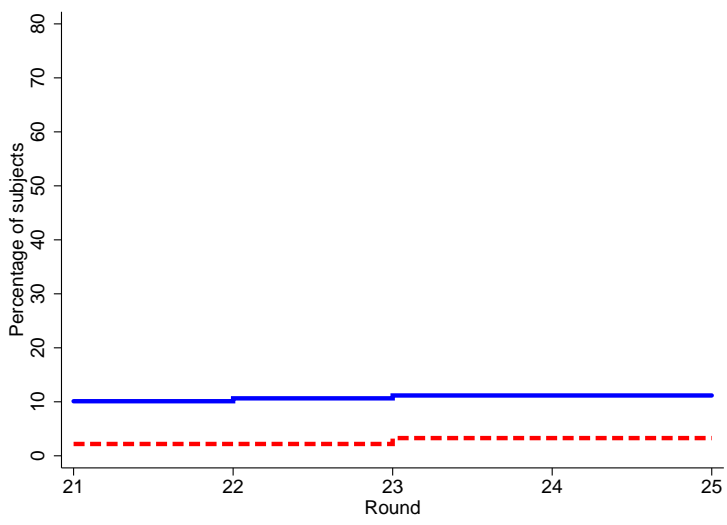

(b) $p=v_{L}$.

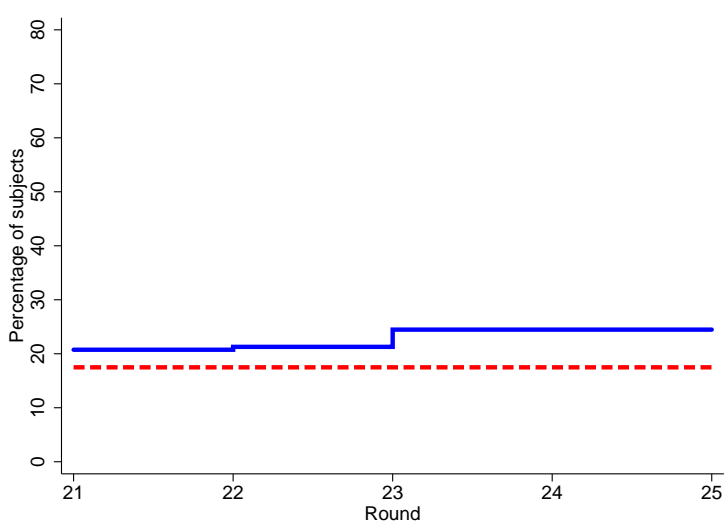

(d) $p \notin\left\{v_{L}, v_{H}\right\}$

Figure 1: Evolution of Types in Part 2.

Notes: Percentage of subjects who submit the described price from round $n$ onwards, for $21 \leq n \leq 24$ by treatment.

Figure 1, in addition, shows that the fraction of subjects classified as one of the aforementioned types does not change much whether we consider subjects who submit a given price in all five rounds or 


\begin{tabular}{cccccc|c}
\hline \hline Part 2 Types & $V_{H}$ & $V_{L}$ & Mix & Dom & Residual & Participants \\
\hline PROB & 47.9 & 10.1 & 8.5 & 24.0 & 9.5 & 188 \\
DET & 65.0 & 2.2 & 7.1 & 20.2 & 5.5 & 183 \\
\hline \hline
\end{tabular}

Table 1: Part 2 Type Classification [as \% of participants]

Notes: Subjects are classified as belonging to a type if their submitted price corresponds to the same type in all 5 rounds $20-25$ in part 2. $V_{L}: p=v_{L} . V_{H}: p=v_{H}$. Mix: $p=v_{L}$ or $p=v_{H}$ (and at least one of each). Dom: $p \notin\left\{v_{L}, v_{H}\right\}$. Residual: all other subjects.

only in the last two rounds. Therefore, we classify subjects as a type if they submit the type-specific price in all five rounds of part 2 , see Table 1.

Table 1 shows that in DET we have substantially more subjects classified as using the dominant strategy $V_{H}$, that is subjects who submit the dominant strategy $p=v_{H}$ in all five rounds, and this difference is significant. ${ }^{21}$ In turn, subjects in PROB are somewhat more likely to be classified as using focal strategies that are dominated, such as $V_{L}$ (submitting $p=v_{L}$ in all five rounds) or Mix (strictly mixing between $p=v_{L}$ and $\left.p=v_{H}\right){ }^{22}$ There are 9.5 and 5.5 percent of subjects who do not follow any of these classifications in PROB and DET, respectively.

\subsection{Part 1 \& Part 2 Behavior}

In part $1, p=v_{L}$ is a dominant strategy for subjects in DET and for subjects in PROB who are not (very) risk seeking. For those latter subjects, dependent on the values of $v_{L}$ and $v_{H}$, a price of $p=v_{H}$ might be utility maximizing. Since subjects are mostly risk averse, and in experiments often very risk averse (see Holt and Laury, 2002), subjects who submit $p=v_{L}$ in part 1 and $p=v_{H}$ in part 2 should comprise almost all subjects who are submitting prices that maximize their payoffs. Thus, we first explore how many of the subjects classified as $V_{H}$ in part 2 also submit $p=v_{L}$ in part 1.

We classify a subject as $V_{L} V_{H}$ if she submitted a price $p=v_{L}$ in the last five rounds of part 1 ( $V_{L}$ in part 1) and $p=v_{H}$ in all five rounds of part $2\left(V_{H}\right.$ in part 2). ${ }^{23}$ The fraction of $V_{L} V_{H}$ subjects in DET (41.5 percent) is more than twice the corresponding fraction in PROB (19.5 percent), confirming the $P_{O} C$ hypothesis.

The vast majority of subjects in both treatments can be classified as using either the payoffmaximizing $V_{L} V_{H}$ strategy, or one of three other alternatives. The second strategy includes behavior

\footnotetext{
${ }^{21}$ To test for the treatment effect we run a linear regression in which the left-hand side variable takes value 1 if the subject is classified as type $V_{H}$ and on the right-hand side we include a treatment dummy $(1=$ DET $)$ and a constant. The treatment effect estimate is 0.17 and the p-value 0.001 .

${ }^{22}$ In part 2, a price $p=v_{L}$ is also dominated, but for expositional purposes we keep that category separate from Dom.

${ }^{23}$ The classification of subjects into types using exclusively the last five periods of part 1 is presented in Table 20 of Appendix B.
} 
that is potentially profit-maximizing in PROB. It requires that subjects are classified as $V_{H}$ in part 2 and, in addition, either strictly mix $v_{H}$ and $v_{L}$ in the last five rounds of part $1\left(M i x V_{H}\right)$, or select $p=v_{H}$ in all the last five rounds of part $1\left(V_{H} V_{H}\right)$. We group subjects who exhibit either behavior in type $\left\{M i x V_{H}, V_{H} V_{H}\right\}$.

In PROB, the $\left\{M i x V_{H}, V_{H} V_{H}\right\}$ type contains 24.5 percent of all subjects, more than the percent classified as $V_{L} V_{H}$. One interpretation is that many of those subjects are risk seeking and profit maximizing. This seems, however, implausible given results in other experiments which often find few subjects to be very risk seeking, as well as for two other reasons. First, there are also many such subjects in DET (15.9 percent), where these strategies are dominated independent of risk preferences. Second, there are other strategies that rely on the subject exclusively submitting prices that are either $v_{L}$ or $v_{H}$ (focal prices), which are not payoff maximizing in either treatment: These consist of part 1/part 2 strategies: $V_{L} V_{L}, V_{L} M i x, M i x V_{L}, M i x M i x, V_{H} V_{L}$, and $V_{H} M i x$, which we group as a third strategy type labeled 'Focal.' In total, 18.7 percent of subjects in PROB are classified as using Focal strategies that cannot be payoff maximizing, compared to 8.3 percent in DET. The prevalence of Focal strategies in PROB therefore suggests that the higher incidence of $V_{H} V_{H}$ and $M i x V_{H}$ types in PROB may be due to a general larger incidence of types in PROB who use only $v_{L}$ and $v_{H}$ prices, though not necessarily in a profit-maximizing way.

In addition, among the Focal strategies, there are two that show an insensitivity towards the fact that values of the companies are changing between part 1 and part 2, namely $V_{L} V_{L}$ and MixMix. Such subjects are almost exclusively present in PROB, where 8.0 and 3.2 percent of participants are classified in these categories compared to 1.1 and 1.6 percent in DET, respectively.

Finally, we also find more subjects in PROB systematically submitting dominated prices that are neither $v_{L}$ or $v_{H}$. This fourth strategy type, which we label 'Dominated,' consists of subjects submitting $p \notin\left\{v_{L}, v_{H}\right\}$ in rounds $16-25{ }^{24}$ There are 21.8 percent of subjects classified as this type in PROB compared to 15.9 percent in DET.

Table 2 presents a summary of the classification of subjects into the four strategy types that use the last five rounds of part 1 and the five rounds of part $2 .{ }^{25}$ Figure 2 shows the evolution the classification if we also include the first fifteen rounds of part 1. For each of the four strategies, each figure reproduces the proportion of types using data from rounds 16-25, but also shows how the proportions would change if, in addition, we demand subjects to follow the corresponding part 1 portion of the strategy not only in the last 5 rounds but starting from round $n \leq 15$.

In particular, Figure 2 a follows the proportion of subjects who are eventually classified as $V_{L} V_{H}$. Notice that the fraction of such profit-maximizing subjects in DET is almost twice that of PROB for any given $n .{ }^{26}$ This is, again, strong indication of the significant role of PoC. At the same time,

\footnotetext{
${ }^{24}$ In part 1 of DET submitting $p=v_{H}$ is also dominated, but for classification purposes and to ease the comparison to PROB we do not consider subjects who consistently submit $p=v_{H}$ in the dominated category.

${ }^{25}$ Subjects classified as 'Residual' submit $p \notin\left\{v_{L}, v_{H}\right\}$ at least once and either $p=v_{L}$ or $p=v_{H}$ at least once in rounds 16-25.

${ }^{26}$ In round 1 the values were selected to match the parametrization of Charness and Levin (2009). In the first round
} 


\begin{tabular}{cccccc|c}
\hline \hline Types & $V_{L} V_{H}$ & $\left\{\right.$ Mix $\left.V_{H}, V_{H} V_{H}\right\}$ & Focal & Dom & Residual & Participants \\
\hline PROB & 19.7 & 24.5 & 18.7 & 21.8 & 15.4 & 188 \\
DET & 41.5 & 15.9 & 8.2 & 15.9 & 18.5 & 183 \\
\hline \hline
\end{tabular}

Table 2: Part 1 and Part 2 Type Classification [as \% of participants]

Notes: Types are defined based on the prices $p_{1}$ submitted in the last five rounds of part 1 and $p_{2}$ submitted in all five rounds of part 2. Type $V_{L} V_{H}: p_{1}=v_{L}$ and $p_{2}=v_{H}$. Type $\left\{M i x V_{H}, V_{H} V_{H}\right\}: p_{1} \in\left\{v_{L}, v_{H}\right\}$ and at least one $p_{1}=v_{H}$ and $p_{2}=v_{H}$. Type 'Focal': $p_{1}, p_{2} \in\left\{v_{L}, v_{H}\right\}$ and at least one $p_{2}=v_{L}$ (corresponds to $V_{L} V_{L}, V_{L}$ Mix, Mix $V_{L}, \operatorname{MixMix}, V_{H} V_{L}$, or $V_{H}$ Mix). Type 'Dom': $p_{1}, p_{2} \notin\left\{v_{L}, v_{H}\right\}$. Residual: All remaining subjects.

there seems to be some learning in both treatments (though there is no feedback): the number of such subjects increases by roughly 50 percent when we move from considering subjects who submit $v_{L}$ from round 1 onwards compared to those who submit $v_{L}$ in the last 5 rounds (and always submit $v_{H}$ in part 2).

In Appendix B, we relax the definition of types in two ways and show that we would reach the same conclusions. First, we classify subjects as a given type even if their prices conform to the type in only four of the last five rounds in part 1 and four of the five rounds in part 2, see Table 21 and Figure 7. Second, allow subjects to submit a price that is slightly higher than the price describing the type of the subject. Although we explicitly asked a question in the instructions, it is possible that some subjects think that they have to bid slightly above $v$ in order to buy the firm. In the second robustness exercise a subject is classified as $V_{L} V_{H}$ if $p \in\left[v_{L}, v_{L}+2\right]$ in the last 5 rounds of part 1 and if $p \in\left[v_{H}, v_{H}+2\right]$ in all rounds of part 2 . See Table 23 for details.

\subsection{Risk Seeking Preferences}

While the previous section already presented evidence suggesting that the prevalence of $p=v_{H}$ prices in PROB is not due to subjects being risk seeking, we now test for this hypothesis directly.

In part 5 subjects chose between three sets of lotteries, where there is a 50-50 chance of obtaining a low $\left(\pi^{L}\right)$ or a high $\left(\pi^{H}\right)$ payoff. For simplicity, we describe a lottery as $\mathcal{L}\left(\pi^{L}, \pi^{H}\right)$. Participants choose between $\mathcal{L}(0,10)$ and $\mathcal{L}(-250,140), \mathcal{L}(0,20)$ and $\mathcal{L}(-180,120)$, and $\mathcal{L}(0,30)$ and $\mathcal{L}(-70,80)$, which correspond to the probabilistic problems $\left\{v_{L}, v_{H}\right\}$ of $\{10,140\},\{20,120\}$, and $\{30,80\}$, if subjects were to submit $p=v_{L}$ and $p=v_{H}$, respectively. ${ }^{27}$ The cases of $\{10,140\}$ and $\{30,80\}$ present the extreme values subjects subjects could have encountered in part 1 , and represent the case where the difference in expected returns between $p=v_{L}$ and $p=v_{H}$ is maximized or

of Charness and Levin's "two-value treatment" 2VT treatment $\{0,99\}, 29.2$ percent of subjects submit a price equal to the low value, 55.7 percent submit a dominated price and 15.1 percent submit a price equal to the high value. In the first round of PROB, 42.0 percent of subjects submit a price equal to the low value, 40.4 percent submit a dominated price, and 18.6 percent submit a price equal to the high value. We have therefore no indication that the Amazon Turk subjects behave differently to the undergraduate students in Charness and Levin (2009).

${ }^{27}$ Recall that subjects were not told that these lotteries corresponded to possible cases of part 1 . All subjects faced these three questions in that same order. 


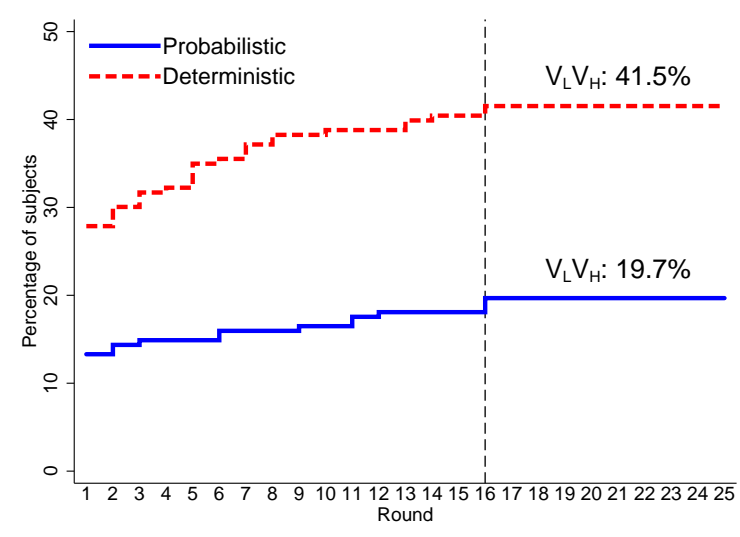

(a) $p=v_{L}$ in $\operatorname{rds} n \leq 15$ and classified as $V_{L} V_{H}$.

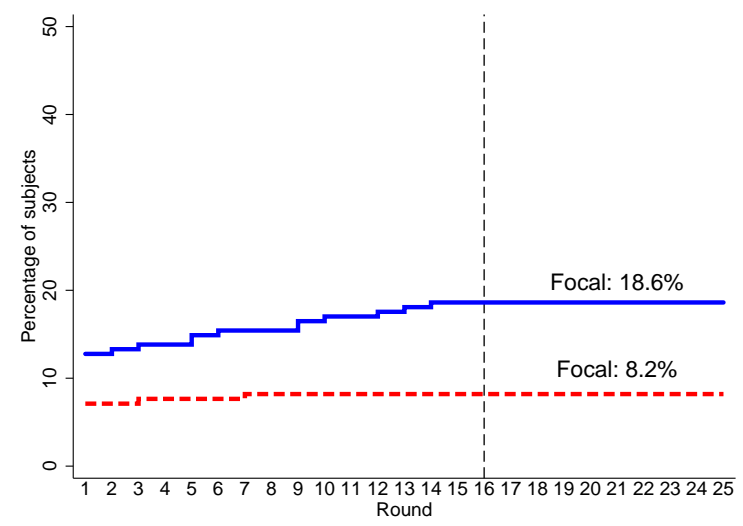

(c) $p \in\left\{v_{L}, v_{H}\right\}$ in $\operatorname{rds} n \leq 15$ and classified as Focal.

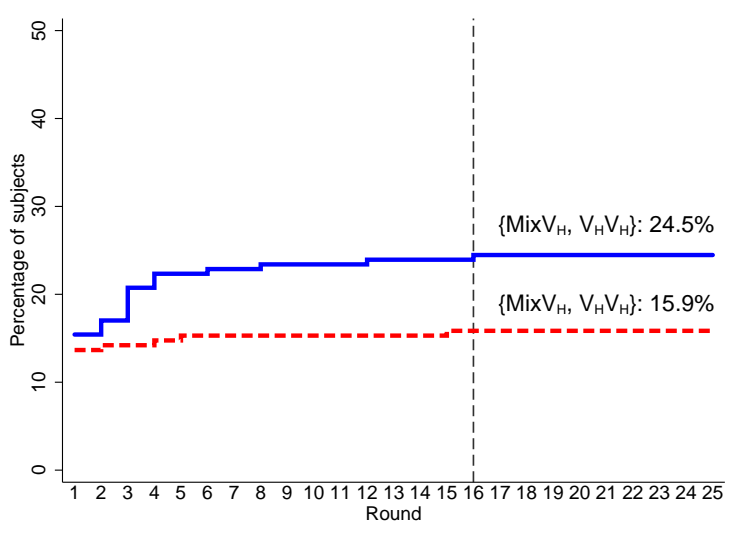

(b) $p=v_{H}$ or strict mix between $v_{L}$ and $v_{H}$ in $\operatorname{rds} n \leq 15$ and classified as $\left\{M i x V_{H}, V_{H} V_{H}\right\}$.

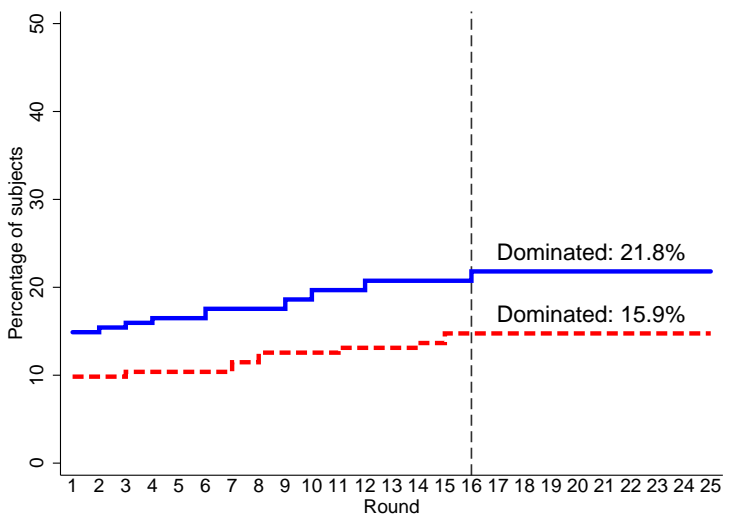

(d) $p \notin\left\{v_{L}, v_{H}\right\}$ in $\operatorname{rds} n \leq 15$ and classified as Dominated.

Figure 2: Evolution of Types (part $1 \&$ part 2). 
minimized, respectively. Overall, 70.0 percent of subjects in DET and 68.6 percent in PROB never took any of the lotteries that correspond to submitting $p=v_{H} \cdot{ }^{28}$

To directly address the role of risk-seeking preferences, Figure 3a shows the fraction of subjects in PROB who submit $p=v_{L}$ from round $n$ until 20 and $p=v_{H}$ in rounds 21-25 (part 2), depending on whether the subject took at least one risky lottery or not. The fact that there is a higher fraction of such subjects among those who who never took a risky lottery compared to those who took at least one risky lottery is consistent with risk-seeking subjects using strategies other than $V_{L} V_{H}$. However, the simultaneous increase of subjects who from round $n$ until round 25 submit prices that correspond to a focal strategy that is never profit maximizing as well as the increase of subjects who consistently use dominated prices, see Figure $3 \mathrm{e}$ and $3 \mathrm{~g}$, is not consistent with subjects who select risky lotteries largely being profit maximizing subjects who are risk seeking.

A second indication that risk-seeking preferences may not be the only and perhaps not even the main driving factor for prices $p=v_{H}$ in part 1 in PROB comes from Figures 3b, 3d, 3f and $3 \mathrm{~h}$ that capture participants in DET. We see changes in the fraction of subjects who use given strategies from round $n$ onwards between subjects who never took a risky lottery and those who took at least one risky lottery in DET that are similar to changes observed in PROB. This is despite the fact that in DET risk preferences have no bearing: submitting a price $p=v_{L}$ in part 1 and $p=v_{H}$ in part 2 is the unique dominant strategy regardless of whether the subject is risk seeking!

\begin{tabular}{lccccc}
\hline & $(1)$ & $(2)$ & $(3)$ & $(4)$ & $(5)$ \\
& $V_{L} V_{H}$ & $\left\{M i x V_{H}, V_{H} V_{H}\right\}$ & Focal & Dom & Dom or Res \\
\hline \multirow{2}{*}{ Det } & $0.244^{* * *}$ & $-0.102^{*}$ & -0.053 & -0.074 & -0.089 \\
& $(0.060)$ & $(0.056)$ & $(0.048)$ & $(0.053)$ & $(0.063)$ \\
Num Risky & $-0.062^{* *}$ & -0.008 & -0.003 & $0.073^{* * *}$ & $0.073^{* *}$ \\
& $(0.031)$ & $(0.029)$ & $(0.025)$ & $(0.027)$ & $(0.033)$ \\
Num Risky $\times$ Det & 0.043 & 0.040 & -0.011 & -0.052 & -0.071 \\
Constant & $(0.046)$ & $(0.043)$ & $(0.036)$ & $(0.040)$ & $(0.048)$ \\
& $0.286^{* * *}$ & $0.223^{* * *}$ & $0.203^{* * *}$ & $0.147^{* *}$ & $0.287^{* * *}$ \\
\hline Observations & $(0.069)$ & $(0.065)$ & $(0.055)$ & $(0.061)$ & $(0.073)$ \\
\hline
\end{tabular}

Table 3: Main Treatments: Estimation output using last 5 rounds of part 1 and part 2 for the classification of types

Notes: Results from a linear regression. The dependent variable takes value 1 if the subject is classified as (1) $V_{L} V_{H},(2)$ $\left\{M i x V_{H}, V_{H} V_{H}\right\}$ (3) Focal, (4) Dom - Dominated, (5) Dom or Res - Dominated or Residual type. Det is a treatment dummy that equals 1 if the subject participated in DET. Num Risky is the number of risky lotteries the subject chooses in part 5 (from 0 to 3). The regression also includes demographic controls (Gender, Ethnicity, Age and Schooling) and a control for the number of errors in the instructions. The full output is presented in Table 28 of the Online Appendix.

Finally, the differences between PROB and DET described in the previous Sections are robust to controls for risk preferences. A linear regression on the classification of subjects (Table 3) shows that

\footnotetext{
${ }^{28}$ The risky alternative was selected only once by 15.4 percent (15.9 percent), twice by 4.8 percent (4.9 percent), and in all three lotteries by 11.2 percent (9.3 percent) of subjects in the PROB (DET) treatment.
} 


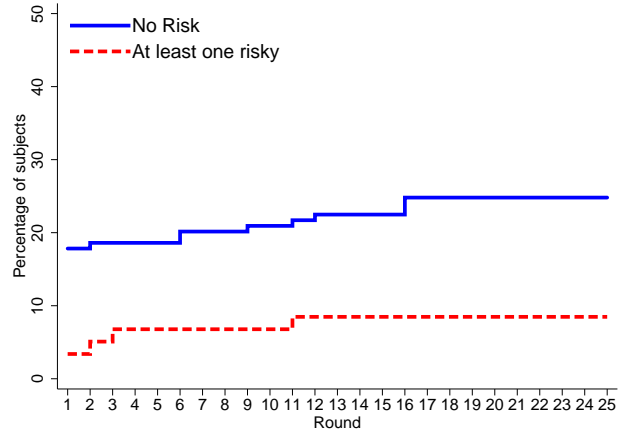

(a) PROB: $p=v_{L}: n$ to $15 \& V_{L} V_{H}$.

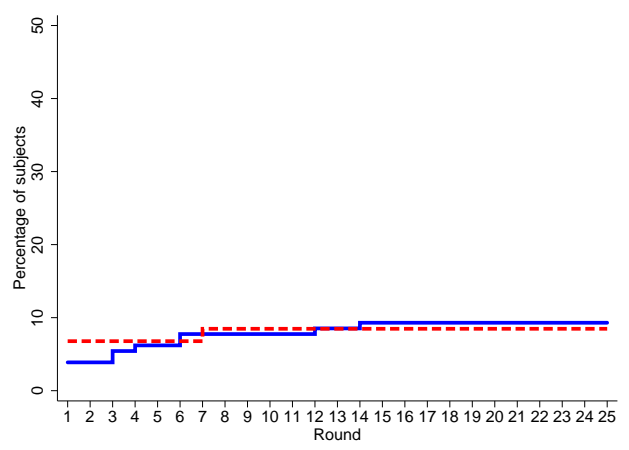

(c) PRoB: $p \in\left\{v_{L}, v_{H}\right\}: \quad n$ to $15 \&$ $\left\{\operatorname{Mix} V_{H}, V_{H} V_{H}\right\}$.

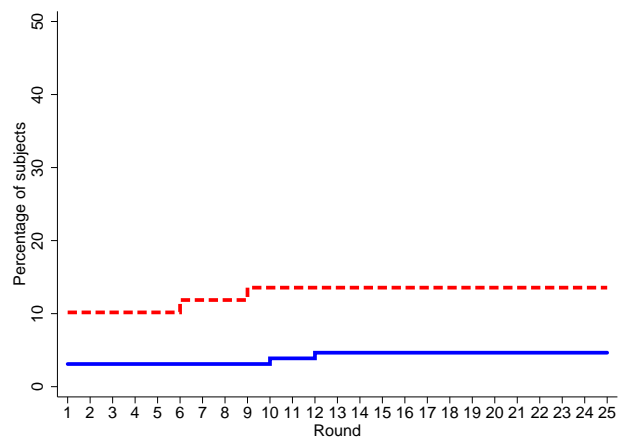

(e) PRoB: $p \in\left\{v_{L}, v_{H}\right\}: n$ to $15 \&$ Focal.

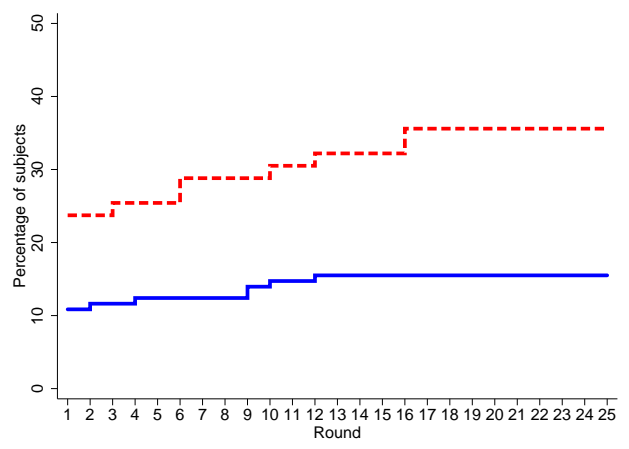

(g) PRoB: $p \notin\left\{v_{L}, v_{H}\right\}: n$ to $15 \&$ Dominated.

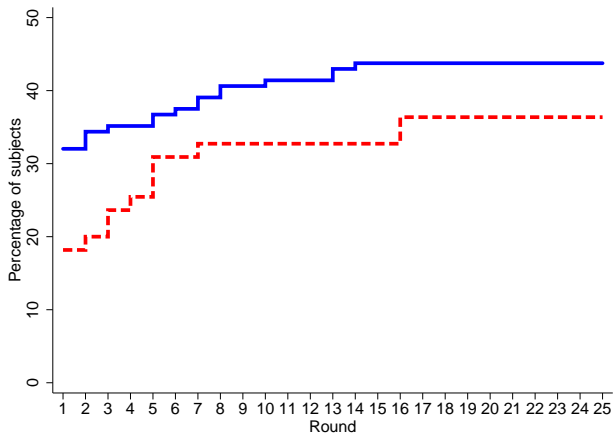

(b) DET: $p=v_{L}: n$ to $15 \& V_{L} V_{H}$.

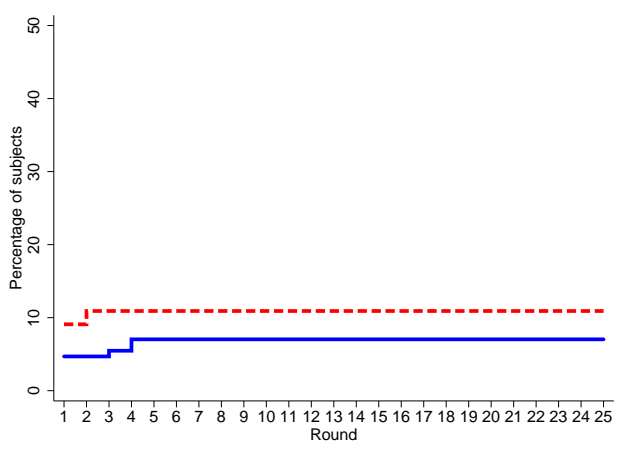

(d) DET: $p \in\left\{v_{L}, v_{H}\right\}: \quad n$ to $15 \&$ $\left\{M i x V_{H}, V_{H} V_{H}\right\}$.

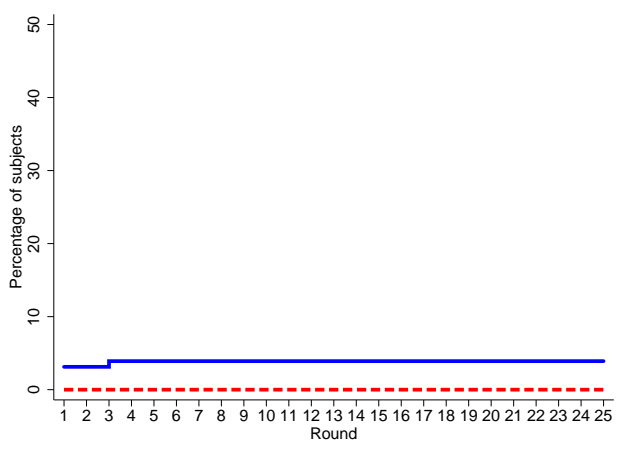

(f) DET: $p \in\left\{v_{L}, v_{H}\right\}: n$ to $15 \&$ Focal.

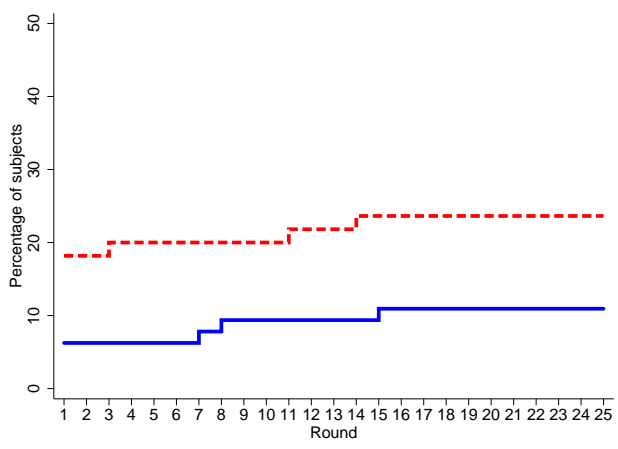

(h) DET: $p \notin\left\{v_{L}, v_{H}\right\}: n$ to $15 \&$ Dominated.

Figure 3: Evolution of Types (Part $1 \&$ Part 2) by Part 5 lottery choices 
there are significantly more subjects classified as $V_{L} V_{H}$ in DET compared to PROB, when controlling for subjects' lottery choices in part 5 .

When we consider the types that consist of strategies that may be dominant for a risk-seeking subject in PROB (though never in DET), namely $V_{H} V_{H}$ and $M i x V_{H}$, they are indeed less likely in DET. However, a final piece of evidence that the number of risky lotteries is not a sign of subjects being very risk seeking but otherwise payoff maximizing is the following: While taking more lotteries significantly reduces the chance for subjects to be classified as $V_{L} V_{H}$, it increases the chance to be classified as the Dominated type. Furthermore, the effect of risk is never significantly different in DET compared to PROB, though risk preferences have no bearing in DET. ${ }^{29}$

To summarize, we do not find evidence for the Risk hypothesis, that is risk-seeking preferences playing a role in accounting for the treatment effect between PROB and DET. This is not surprising given that individuals are in general classified as risk averse, and a subject in part 1 would have to be quite risk seeking for $p=v_{L}$ not being the profit-maximizing price in PROB as well as in DET.

\subsection{The Role of the Power of Certainty}

While in the previous subsections we provided evidence for $P_{o} C$, in this subsection we address the relative importance of $P o C$ in accounting for the difficulties when moving from an environment involving a decision for a single known contingency to an environment with several contingencies that are uncertain. That is, what fraction of the problem is due to the fact that the number of contingencies increases from one to two (Complexity) and what fraction is due to the fact that the two contingencies are uncertain rather than certain $\left(P_{o} C\right)$ ? Put differently, when comparing the behavior in PROB to the behavior of subjects in the One-Firm treatment: How much of the difference between the two treatments can be accounted for by DET? If PoC plays a quantitatively significant role, then DET should not be almost identical to PROB, but rather be different from PROB and moving towards outcomes in ONEFIRM.

We first describe results of the 25 rounds in the One-Firm treatment, where the problem consists of a single firm of known value. Note that we find no statistical difference depending on whether subjects were introduced to the one-firm environment using probabilistic or deterministic instructions. ${ }^{30}$ Consequently, we pool all data from both treatments and we label the joint treatment as ONEFIRM. When we classify subjects based on the prices they submit in the last 10 rounds, 62.6 percent submit only $p=v$, a strategy type that we refer to as $V .{ }^{31}$ A total of 13.6 percent of subjects

\footnotetext{
${ }^{29}$ While in this and all other regressions we use the number of risky lotteries subjects chose, our results are robust to instead using a dummy indicating whether a subject chose at least one risky lottery, see Table 24 in Appendix C.

${ }^{30}$ We run a panel regression in which the left-hand side is a dummy variable $(1$ if $p=v)$ and the right-hand side includes a treatment dummy ( 1 if the subject was introduced to the one-firm problem after reading deterministic instructions), demographic controls and a constant. The estimated treatment dummy is quite small and not significant in the whole sample $(-0.012, \mathrm{p}$-value $=0.717)$ or if we constrain the sample to prices in the last 10 rounds $(0.005$, $\mathrm{p}$-value $=0.883)$.

${ }^{31}$ Appendix A describes the aggregate distribution of prices that subjects submitted in this treatment when there is no constraint of consistency within subject.
} 


\begin{tabular}{ccccccc|c}
\hline \hline & & $V_{L} V_{H} / V$ & $\left\{M i x V_{H}, V_{H} V_{H}\right\}$ & Focal & Dom & Residual & Participants \\
\hline \multirow{4}{*}{ All } & PROB & 19.7 & 24.5 & 18.6 & 21.8 & 15.4 & 188 \\
& DET & 41.5 & 15.9 & 8.2 & 15.9 & 18.6 & 183 \\
& ONEFIRM & 62.6 & - & - & 13.6 & 23.8 & 428 \\
\hline \hline No & PROB & 24.8 & 25.6 & 18.6 & 15.5 & 15.5 & 129 \\
Risky & DET & 43.8 & 15.6 & 9.4 & 11.7 & 19.5 & 128 \\
Lottery & ONEFIRM & 67.8 & - & - & 13.4 & 18.8 & 276 \\
\hline \hline At least one & PROB & 8.5 & 22.0 & 18.6 & 35.6 & 15.3 & 59 \\
Risky & DET & 36.4 & 16.4 & 5.5 & 25.5 & 16.4 & 55 \\
Lottery & ONEFIRM & 53.3 & - & - & 13.8 & 32.9 & 152 \\
\hline \hline
\end{tabular}

Table 4: Type classification using rounds 15-25 [as \% of participants]

Notes: Types are defined based on the prices $p_{1}$ submitted in the last five rounds of part 1 and $p_{2}$ submitted in all five rounds of part 2. Type $V_{L} V_{H}: p_{1}=v_{L}$ and $p_{2}=v_{H}$. Type $\left\{M i x V_{H}, V_{H} V_{H}\right\}: p_{1} \in\left\{v_{L}, v_{H}\right\}$ and at least one $p_{1}=v_{H}$ and $p_{2}=v_{H}$. Type 'Focal': $p_{1}, p_{2} \in\left\{v_{L}, v_{H}\right\}$ and at least one $p_{2}=v_{L}$ (corresponds to $V_{L} V_{L}, V_{L}$ Mix, Mix $V_{L}, \operatorname{MixMix}, V_{H} V_{L}$, or $V_{H}$ Mix). Type 'Dom': $p_{1}, p_{2} \notin\left\{v_{L}, v_{H}\right\}$. Residual: All remaining subjects. In onEFIRM subjects are classified as $V$ if they submit $p=v$ in rounds 16-25, and as 'Dom' if $p \neq v$ in rounds 16-25, with Residual being the remaining subjects.

submit dominated prices: 8.6 percent submit only $p>v, 1.2$ percent submit only $p<v$, and 3.8 percent submit either $p<v$ or $p>v$ in the last 10 rounds. Subjects who submit each, a dominated price and $p=v$ in at least one of the last 10 rounds are classified as the Residual type, which covers 23.8 percent, see Table $4 .^{32}$

If ONEFIRM, where there is only one firm of known value, is a benchmark for the number of subjects who consistently make profit-maximizing choices, then 62.6 percent of subjects consistently do so in rounds 16-25. In contrast, when there is one firm of two possible values, both equally likely, as in PROB, only 19.7 percent of subjects submit the dominant strategy (for not very risk-seeking subjects) in rounds 16-25, namely $p=v_{L}$ in rounds $16-20$ and $p=v_{H}$ in rounds 21-25. One interpretation is that among the subjects who consistently submit profit-maximizing prices in ONEFIRM, only 31.5 percent are able to do so when the firm has two possible values each with 50 percent chance. ${ }^{33}$

Put differently the 42.9 percentage points reduction in subjects who submit profit-maximizing strate-

\footnotetext{
${ }^{32}$ If we relax the definition and allow for two periods (out of the last 10) in which the subject did not submit $p=v$, the proportion of subjects classified as $V$ increases from 62.6 to 67.1 percent. Likewise, if we allow for at most two periods in which the subject did not submit a dominated choice, the proportion of subjects submitting dominated choices increases from 13.6 to 20.5 percent. Table 21 in Appendix B presents further details. In addition, Table 23 in Appendix B shows the results if we relax the definition allowing for optimality being defined as the subject submitting $p \in[v, v+2]$. That is, we consider a choice to be optimal if the overbidding is at most two units above $v$. The proportion of subjects classified as $V$ increases to 66.8 percent.

${ }^{33}$ For a qualitative comparison, we summarize the corresponding findings of Esponda and Vespa (2014). In their sequential voting treatment (in which subjects can perfectly infer the state before making a decision) approximately 76 percent of subjects consistently make the optimal decision. However, when subjects subsequently make a decision in which the state is unknown (but in which subjects do know the probability distribution over the states) approximately 22 percent of subjects make the optimal decision. Even though the environment in Esponda and Vespa (2014) is quite different from ours, and they use NYU students rather than Amazon Turk workers, the quantitative effects are surprisingly similar.
} 
gies is an upper bound of the size of the problem created by the computational Complexity hypothesis. We use DET to assess the relative impact of PoC. In DET, 41.5 percent of subjects consistently use profit-maximizing strategies. That is 21.8 percentage points of the problem, slightly more than half of the total effect, is driven by probabilistic difficulties, the lack of $P_{o} C$, and only the remaining half can be attributed to pure computational complexity, the fact that subjects have to deal with multiple (two) contingencies rather than just one.

To ensure that risk-seeking preferences do not distort results, the second set of rows of Table 4 shows the classification of subjects who never took any risky lottery and hence are not very risk seeking. Among those subjects, the strategy $V_{L} V_{H}$ is the only dominant strategy. The difference between ONEFIRM and PROB for this type is 43 percentage points. Once more, the proportion of subjects in DET who submit profit-maximizing strategies is roughly in between ONEFIRM and PROB. Of the 43 percentage point difference, a simple decomposition suggests that 24 percentage points are related to computational complexity, and 19 percentage points to the fact that there is uncertainty in PROB.

An alternative approach to evaluate the relative role of $P o C$ is to measure differences between treatments in the payoff space. The first measure we provide is simply the payoffs subjects achieve, where for PROB we compute expected payoffs. Note that to compare the earnings to the ONEFIRM benchmark in part 1 problems, we restrict attention to ONEFIRM cases where the realized value of the firm was $v_{L}$ and we simply use the submitted price $p$ to compute earnings as $1.5 \times v_{L}-p$ (since if we were to use values of $v_{H}$ in part 1 there would be profits in ONEFIRM that are simply not achievable by the dominant strategy in DET or - for risk-neutral subjects - in PROB). ${ }^{34}$ Averaging earnings over all 25 rounds of part 1 and part 2, of the total payoff gain for the median subject in ONEFIRM relative to PROB, 69.4 percent is achieved by the median subject in the DET. That is, for the median subject, $P o C$ accounts for approximately seventy percent of the payoff loss incurred by contingent reasoning. ${ }^{35}$

Instead of using realized prices, we can ask which fraction of gains a subject made when comparing their payoffs to, on the one hand, random behavior, and, on the other hand, optimal behavior. Specifically, we compute a relative payoff variable in which the numerator is the subject's profit minus the profit that would result from random prices and the denominator is the difference between the profit of the price-maximizing action for a risk-neutral subject and the profit that results from

\footnotetext{
${ }^{34}$ Specifically, in ONEFIRM subjects saw 20 randomly drawn numbers of the 40 values that correspond to the $20 v_{L}$ and $20 v_{H}$ values in part 1 . For each subject we use only values of $v$ that correspond to a value of some $v_{L}$ in part 1. That is, there is a chance that some subjects had two values of $v_{L}=28$, while subjects in PROB or DET only saw one value of $v_{L}=28$. To compute the profits in ONEFIRM of part 2 , we consider only values $v$ that correspond to a value of $v_{H}$ in part 2. For a given value of $v_{H}$ there might be several values of $v_{L}$ that are coupled to it in part 2: For example, there are two questions for which $v_{H}=54$. One with $v_{L}=48$ and another with $v_{L}=50$. We assign to half the cases of $v=54$ a value $v_{L}=48$ and the other half a value of $v_{L}=50$. The profits in a given round of part 2 of the subject in ONEFIRM is computed whenever $v=v_{H}$ and the profit is $\left(1.5 \times v_{L}-p\right)+\left(1.5 \times v_{H}-p\right)$, where $v_{L}$ is one of the values associated with $v_{H}$ in part 2 of PROB or DET. For each part, we compute average profits by round given the relevant choices as described above.

${ }^{35}$ If we constrain the sample to subjects who never took a risk in part 5 the number is 58.4 percent. This is because, particularly in $\mathrm{PROB}$, subjects who take risks in part 5 are more likely to obtain lower earnings in part 1 than those who never took a risky lottery.
} 
random prices. ${ }^{36}$ Of the total relative payoff gains for the median subject in ONEFIRM relative to PROB, 71.4 percent is achieved by the median subject in DET. ${ }^{37}$

As a final approach we use the lottery choices in part 5 as a proxy for the subject's optimal choice in part 1 (in part 2 the optimal choice is $p=v_{H}$ ). Specifically, the lottery choice is a reduction or simplification of a problem akin to those in part 1 in PROB, as the two lotteries correspond to the lotteries that result from $p=v_{L}$ and $p=v_{H}$, respectively. We therefore assume that choices in this simplified "probabilistic problem" are a better predictor of underlying preferences over outcomes than the choice of a price in the more complicated probabilistic problem of part 1 (see Ambuehl, Bernheim and Lusardi, 2014 for a similar approach). That is, we consider that $p=v_{L}$ captures the welfare-maximizing choice for subjects in DET as well as for any subject in PROB who always selected the safer lottery in part 5 (which corresponds to submitting $p=v_{L}$ ). For subjects in PROB who took some risks in part 5 , the optimal choice can be either $v_{L}$ or $v_{H}$, where for $p<v_{H}$ we use $v_{L}$ and for $p \geq v_{H}$ we use $v_{H}$. For subjects in PROB who selected the risky lottery in all three questions of part 5 we assume that $v_{H}$ is the optimal choice. Finally, $p=v$ is considered optimal in the ONEFIRM treatments.

Note that for some subjects, the imputed welfare maximizing choice in PROB does not yield the highest expected earnings. We therefore cannot simply aggregate earnings. Rather, we ask how often a subject made the imputed welfare-maximizing choice. We then compute the gain in terms of the fraction of welfare-maximizing choices for the median subject in ONEFIRM relative to the median subject in PROB. We find that 39.3 percent of the increase in that fraction is achieved by the median subject in DET. If we restrict the sample to subjects who never took a risky lottery in Part 5, 50 percent of the increase in the fraction of welfare-maximizing choices is achieved by the median subject in DET. ${ }^{38}$

To summarize, we find that in both the strategy space and the payoff space, there is robust evidence that a substantial part of the problem of contingent reasoning can be attributed to the lack of $P o C$ rather than pure computational complexity. More concretely, there is an important improvement, both in the action and payoff space, when we compare the two contingency environment of DET relative to the two contingency environment of $\mathrm{PROB}$, where a major difference is whether the two contingencies are certainties.

\footnotetext{
${ }^{36}$ To compute the payoff from random choice, we draw, for each question, 1000 uniform values in $[0,150]$ and for each draw compute the payoff that would result if the draw were the submitted price. We then average across the 1000 draws. We therefore have for each question an optimal, a random and an actual payoff measure. We then compute, for each question, (Actual Payoff - Random)/(Optimal - Random). Since Actual Payoff could be smaller than Random (if the subject submitted, e.g. 150), note that this measure is sometimes negative. After computing the ratio for each question, we take the average at the subject level.

${ }^{37}$ If we constrain the sample to subjects who never took a risky lottery in part 5 the number is 64.7 percent.

${ }^{38}$ Note that when we use the measure consisting of the fraction of welfare-maximizing choices, the role of $P o C$ is increased if we restrict attention to subjects who never took a risky lottery in part 5 . This is because using lottery choices to impute welfare-maximizing choices, allowed for some subjects in PROB to submit $p=v_{H}$ in part 1 and still make a welfare-maximizing choice. This is not the case when we restrict attention to subjects who never took a risky lottery, thereby reducing the fraction of welfare-maximizing choices of the median subject in PROB and as such increasing the effect of $P o C$.
} 


\begin{tabular}{cccc}
\hline \hline & & PROB & DET \\
\hline \multirow{4}{*}{ All Outcomes } & Both Explicit & 1.6 & 13.7 \\
& One Explicit & 0.0 & 8.7 \\
& Qualitative & 14.4 & 30.1 \\
& Mention Outcomes Only & 10.6 & 0.0 \\
\hline \hline
\end{tabular}

Table 5: Reported Payoff in Part 3 Advice for Subjects who mention All Outcomes

Notes: 188 participants in PROB and 183 in DET. There are four outcomes $(v, p)$ where $v, p \in\left\{v_{L}, v_{H}\right\}$. The four rows divide subjects who mention all four outcomes dependent on whether subjects explicitly report payoffs associated with $p=v_{L}$ and (or) $p=v_{H}$ : Both (One) Explicit; report payoffs qualitatively (Qualitative) or only mention all outcomes without addressing payoffs (Mention Outcomes Only).

\section{Understanding the Power of Certainty}

In this section we shed light on the underlying cause of $P o C$. We first analyze the advice subjects provided to a different participant. Specifically, we assess whether subjects differ in mentioning the four possible outcomes associated with submitting a price $p=v_{L}$ and $p=v_{H}$. We then explore to what extent differences in advice can account for differences in strategies between PROB and DET. In a final treatment we explore the robustness of $P o C$. We ask whether having subjects first encounter the ONEFIRM problem, and hence be attuned to thinking about the value of the firm, increases the propensity to use dominant strategies and reduces differences in choices between probabilistic and deterministic problems.

\subsection{Advice}

To shed light on the underlying cause for the $P o C$ hypothesis, we analyze the advice subjects provide to another participant for the $v_{L}=20, v_{H}=120$ problem. In both PROB and DET the vast majority of subjects provide a numerical advice (90.4 and 92.4 percent) and/or some explanation (77.7 and 79.2 percent, respectively). Furthermore, the length of the written advice is, while similar between PROB and DET, if anything a little longer in PROB. ${ }^{39}$

The overall pattern of numerical recommendations matches our earlier findings. While 63.9 percent of subjects in DET recommend submitting $p=v_{L}, 43.1$ percent submit the same recommendation in PROB (Table 25 of Appendix D). Meanwhile, 32.5 percent of subjects in PROB recommend to either submit $p=v_{H}$ or mix between $v_{L}$ and $v_{H}$, compared to 17.5 percent in DET.

\footnotetext{
${ }^{39}$ The median (mean) number of words used in the advice equals 42 (54.7) in PROB and 34 (45.1) in DET. Using a quantile regression on the median (linear regression) with a treatment dummy on the right-hand side and the number of words on the left-hand side we find that the difference in the median (mean) is significant at the 10 (5) percent level (p-values of 0.06 and 0.03 , respectively).
} 


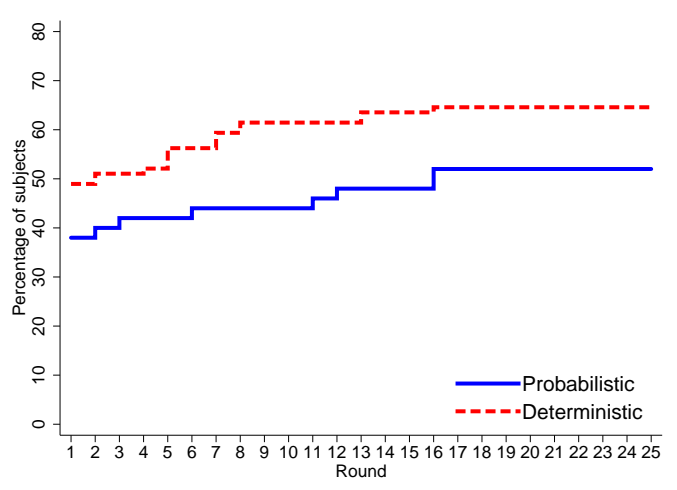

(a) Subjects whose Advice mentions all Four Outcomes

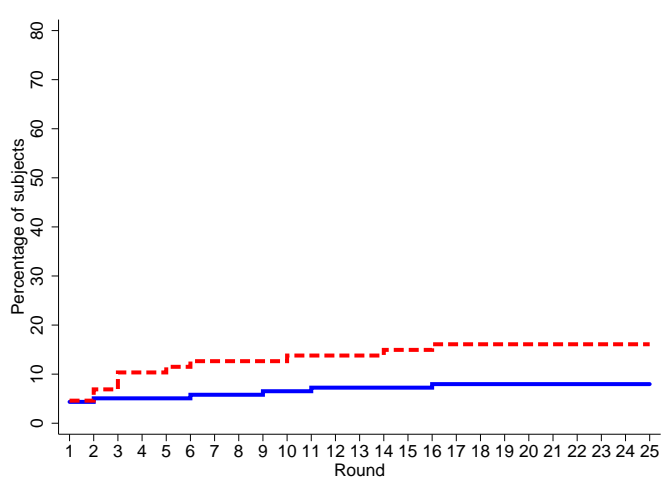

(b) Subjects whose Advice does not mention all Four Outcomes

Figure 4: Percent of subjects with $p=v_{L}$ in round $n \leq 15$ and classified as $V_{L} V_{H}$ in PROB and DET dependent on whether their advice mentions all four outcomes.

In general, to determine the payoff-maximizing price, a subject who understood that submitting prices $p \notin\left\{v_{L}, v_{H}\right\}$ is dominated needs to be aware of the four outcomes $(v, p)$ where $v, p \in$ $\left\{v_{L}, v_{H}\right\} .{ }^{40}$ Table 5 shows that 26.6 percent of subjects in PROB mention all four outcomes in at least some way compared to 52.5 percent in DET, a significant difference. ${ }^{41}$ Only 3 subjects $(1.6$ percent) in PROB explicitly compute payoffs for both $p=v_{L}$ and $p=v_{H}$, compared to 13.7 percent of subjects in DET. An additional 8.7 percent of subjects in DET compute the payoff associated with either $p=v_{L}$ or $p=v_{H}$. More subjects in DET than in PROB qualitatively mention all four outcomes. There are some subjects in PROB who mention all four outcomes without, however, providing any guidance as to how to use these outcomes to compute payoffs.

Figure 4 shows the fraction of subjects who submit $p=v_{L}$ from round $n$ to 20 for $1 \leq n \leq 16$ and submit $p=v_{H}$ in rounds 21-25 (part 5) in PROB and DET, dependent on whether the subject did (Figure 4a) or did not (Figure 4b) mention all four outcomes. The Figure suggests that mentioning all four outcomes increases incidences of submitting the strategy $V_{L} V_{H}$. It also suggests that within each of those two groups the treatment effect is smaller.

Table 6 shows the distribution of types in each treatment depending on whether subjects mention all four outcomes, and suggests that this feature of the advice may account for differences across treatments. For example, the difference in the fraction of $V_{L} V_{H}$ types is approximately 12 percentage points among subjects who mention all four outcomes, which is close to 50 percent of the original difference. This suggests that differences across treatments might be driven by differences in incidences of advice that mentions all four outcomes. The table also suggests that subjects who do not mention all four outcomes are much more likely to be classified as a Dominated or Residual

\footnotetext{
${ }^{40}$ The advice was classified following the protocol described in Section B of the Online Appendix. The classification was verified by a research assistant. Examples of each class of advice are provided in Appendix F.

${ }^{41}$ To test for significance, we run a linear regression in which the left-hand side variable is a dummy that takes value 1 if the subject submitted advice that mentions all four outcomes and on the right-hand side we include a constant and a treatment dummy $(1=\mathrm{DET})$. The coefficient on the treatment dummy is positive and significant at the 1 percent level (p-value $<0.01)$
} 


\begin{tabular}{ccccccc|c}
\hline \hline & & $V_{L} V_{H}$ & $\left\{\right.$ Mix $\left._{H}, V_{H} V_{H}\right\}$ & Focal & Dom & Residual & Participants \\
\hline \hline All four & PROB & 52.0 & 20.0 & 22.0 & 4.0 & 2.0 & 50 \\
Outcomes & DET & 64.6 & 7.3 & 7.3 & 7.3 & 13.6 & 96 \\
\hline Not all four & PROB & 8.0 & 26.1 & 17.4 & 28.3 & 20.3 & 138 \\
Outcomes & DET & 16.1 & 25.3 & 9.2 & 25.3 & 24.1 & 87 \\
\hline \hline
\end{tabular}

Table 6: Participants classified into types using rounds 15-25 (the last 5 rounds in part 1 and in part 2) [as \% of participants]

Notes: Types are defined based on the prices $p_{1}$ submitted in the last five rounds of part 1 and $p_{2}$ submitted in all five rounds of part 2. Type $V_{L} V_{H}: p_{1}=v_{L}$ and $p_{2}=v_{H}$. Type $\left\{M i x V_{H}, V_{H} V_{H}\right\}: p_{1} \in\left\{v_{L}, v_{H}\right\}$ and at least one $p_{1}=v_{H}$ and $p_{2}=v_{H}$. Type 'Focal': $p_{1}, p_{2} \in\left\{v_{L}, v_{H}\right\}$ and at least one $p_{2}=v_{L}$ (corresponds to $V_{L} V_{L}, V_{L} \operatorname{Mix}, \operatorname{Mix} V_{L}$, MixMix, $V_{H} V_{L}$, or $V_{H} M i x$. Type 'Dom': $p_{1}, p_{2} \notin\left\{v_{L}, v_{H}\right\}$. Residual: All remaining subjects.

type.

Table 7 confirms that subjects who mention all four outcomes are more likely to be classified as $V_{L} V_{H}$ and less likely to be classified as using a strategy dominated in all treatments that is not focal. This suggests that mentioning all four outcomes $(v, p)$ where $v, p \in\left\{v_{L}, v_{H}\right\}$ - and hence thinking about them - is important to using the dominant strategy and to avoid consistently using prices that are dominated and not focal $\left(p \notin\left\{v_{L}, v_{H}\right\}\right)$. The effect of mentioning all four outcomes on type classification does not significantly differ by treatment. Furthermore, the differences between PROB and DET are no longer significant when we condition on whether subjects mentioned all four outcomes in the advice.

The results suggests that a big part of $P o C$ stems from helping subjects to envision all four outcomes. In contrast, when outcomes are hypothetical and not actually realized and certain, subjects seem to have a much harder time to think of all four outcomes.

To further our understanding of what subjects may think about when they do not mention all four outcomes, we divide subjects into five categories (shown in Table 8). The first is subjects who do not mention any of the four outcomes. Another consists of subjects who only mention outcomes $(v, p)$ associated with $p=v_{L}$, that is either only $\left\{\left(v_{L}, v_{L}\right)\right\}$ or only $\left\{\left(v_{L}, v_{L}\right)\right.$ and $\left.\left(v_{H}, v_{L}\right)\right\} .{ }^{42}$ We classify subjects as mentioning large gains, if they mention or highlight the gains that can be obtained for a firm of value $v=v_{H}$ when submitting a price of $p=v_{H} \cdot{ }^{43}$ We classify subjects as mentioning large losses, if they mention or highlight the losses that can result for a firm of value $v=v_{L}$ when submitting a price of $p=v_{H} \cdot{ }^{44}$ Subjects in PROB are more likely not to mention any outcome

\footnotetext{
${ }^{42}$ Some of these latter subjects are subjects who compute the payoff associated with $p=v_{L}$.

${ }^{43}$ These are subjects who mention only $\left\{\left(v_{L}, v_{L}\right)\right.$ and $\left.\left(v_{H}, v_{H}\right)\right\}$, only $\left\{\left(v_{H}, v_{H}\right)\right\}$, only $\left\{\left(v_{L}, v_{H}\right)\right.$ and explicitly $\left.\left(v_{H}, v_{H}\right)\right\}$ or only $\left\{\left(v_{L}, v_{L}\right),\left(v_{H}, v_{L}\right)\right.$ and $\left.\left(v_{H}, v_{H}\right)\right\}$. We consider an outcome to be mentioned if it is mentioned explicitly or implicitly. An example in which $\left(v_{H}, v_{H}\right)$ is only implicitly mentioned would be the following: "submitting 120 can lead to a gain, but it's not worth the risk." In this case we consider that the subject is implicitly mentioning that if $v=v_{H}$, there would be a gain.

${ }^{44}$ These are subjects who mention only $\left\{\left(v_{L}, v_{H}\right)\right\}$, only $\left\{\left(v_{L}, v_{H}\right)\right.$ and implicitly $\left.\left(v_{H}, v_{H}\right)\right\}$, only $\left\{\left(v_{L}, v_{L}\right),\left(v_{H}, v_{L}\right)\right.$ and $\left.\left(v_{L}, v_{H}\right)\right\}$ or only $\left\{\left(v_{L}, v_{L}\right),\left(v_{L}, v_{H}\right)\right.$ and $\left.\left(v_{H}, v_{H}\right)\right\}$.
} 


\begin{tabular}{|c|c|c|c|c|c|}
\hline & $\begin{array}{c}(1) \\
V_{L} V_{H}\end{array}$ & $\begin{array}{c}(2) \\
\left\{M i x V_{H}, V_{H} V_{H}\right\}\end{array}$ & $\begin{array}{l}(3) \\
\text { Focal }\end{array}$ & $\begin{array}{c}(4) \\
\text { Dom }\end{array}$ & $\begin{array}{c}(5) \\
\text { Dom or Res }\end{array}$ \\
\hline Det & $\begin{array}{c}0.117 \\
(0.073)\end{array}$ & $\begin{array}{c}0.021 \\
(0.074)\end{array}$ & $\begin{array}{c}0.004 \\
(0.064)\end{array}$ & $\begin{array}{c}-0.084 \\
(0.070)\end{array}$ & $\begin{array}{r}-0.142^{*} \\
(0.082)\end{array}$ \\
\hline Advice mentions all outcomes & $\begin{array}{l}0.395^{* * *} \\
(0.068)\end{array}$ & $\begin{array}{c}-0.088 \\
(0.069)\end{array}$ & $\begin{array}{c}0.062 \\
(0.059)\end{array}$ & $\begin{array}{l}-0.184^{* * *} \\
(0.065)\end{array}$ & $\begin{array}{l}-0.369^{* * *} \\
(0.076)\end{array}$ \\
\hline Advice mentions all outcomes $\times$ Det & $\begin{array}{c}0.025 \\
(0.092)\end{array}$ & $\begin{array}{c}-0.148 \\
(0.093)\end{array}$ & $\begin{array}{c}-0.112 \\
(0.080)\end{array}$ & $\begin{array}{c}0.090 \\
(0.088)\end{array}$ & $\begin{array}{l}0.234^{* *} \\
(0.103)\end{array}$ \\
\hline Num Risky & $\begin{array}{c}-0.029 \\
(0.029)\end{array}$ & $\begin{array}{c}-0.015 \\
(0.029)\end{array}$ & $\begin{array}{c}0.003 \\
(0.025)\end{array}$ & $\begin{array}{l}0.058^{* *} \\
(0.028)\end{array}$ & $\begin{array}{c}0.042 \\
(0.032)\end{array}$ \\
\hline Num Risky $\times$ Det & $\begin{array}{c}0.004 \\
(0.042)\end{array}$ & $\begin{array}{c}0.050 \\
(0.043)\end{array}$ & $\begin{array}{r}-0.016 \\
(0.037)\end{array}$ & $\begin{array}{c}-0.036 \\
(0.040)\end{array}$ & $\begin{array}{c}-0.039 \\
(0.047)\end{array}$ \\
\hline Constant & $\begin{array}{r}0.132^{*} \\
(0.069)\end{array}$ & $\begin{array}{l}0.257^{* * *} \\
(0.070)\end{array}$ & $\begin{array}{l}0.178^{* * *} \\
(0.060)\end{array}$ & $\begin{array}{l}0.220^{* * *} \\
(0.066)\end{array}$ & $\begin{array}{l}0.433^{* * *} \\
(0.077)\end{array}$ \\
\hline Observations & 371 & 371 & 371 & 371 & 371 \\
\hline
\end{tabular}

Table 7: Main Treatments: Estimation output using last 5 rounds of part 1 and part 2 for the classification of types

Notes: Results from a linear regression. The dependent variable takes value 1 if the subject is classified as (1) $V_{L} V_{H},(2)$ $\left\{M i x V_{H}, V_{H} V_{H}\right\}$ (3) Focal, (4) Dom, (5) Dom or Res. Det is a treatment dummy that equals 1 if the subject participated in DET. Advice mentions all outcomes is a dummy that equals 1 if the advice of the subject mentions all four outcomes $(v, p)$ with $v, p \in\left\{v_{L}, v_{H}\right\}$. Num Risky is the number of risky lotteries the subject chooses in part 5 (from 0 to 3 ). The regression also includes demographic controls (Gender, Ethnicity, Age and Schooling) and a control for the number of errors in the instructions. The full output is presented in Table 35 of the Online Appendix.

compared to subjects in DET (by about thirty percent). However, subjects in PROB are much more likely to only report a subset of the outcomes, either focusing on large gains or large losses: a total of 29.3 percent of subjects compared to 14.2 percent in DET.

The advice subjects provide is a strong indicator of the recommended price and the strategy they use, see also Appendix D tables 25 and 26 for details. Subjects who mention all four outcomes are to a large extent recommending $p=v_{L}$ in part 1 (76.0 and 97.9 percent in PROB and DET, respectively). ${ }^{45}$ This is much larger than the 31.2 and 26.4 percent among all other subjects (a two-sided Fisher's exact test yields $p<0.01$ and $p<0.01$, respectively). We have already shown that subjects who mention all four outcomes are more likely to be classified as $V_{L} V_{H}: 52.0$ and 64.6 percent in PROB and DET, respectively, compared to only 8.0 and 16.1 percent among all other subjects $(p<0.01$ and $p<0.01$, respectively).

To assess the impact of mentioning only large gains or only large losses, we exclude from our sample subjects who mention all four outcomes (as we already have a pretty good understanding of their behavior). Subjects who mention only large gains are very likely to recommend $p=v_{H}(83.3$ and 81.8 percent) in PROB and DET, respectively. This is larger than the 20.2 and 30.3 percent among subjects who do not mention only large gains - and neither all four outcomes $(p<0.01$ and $p<0.01$,

\footnotetext{
${ }^{45}$ Of subjects who mention all four outcomes in PROB, there are 3 subjects (1.6 percent) who recommend $p=120$ (for details see Table 25). One of these three subjects explicitly computes the payoffs for the four outcomes, and in part 5 selects the lottery that corresponds to $p=120$ when facing a choice between the lotteries induced by $p=20$ and $p=120$. The other two subjects who recommend $p=120$ only describe the four outcomes qualitatively, and when they face the lotteries in part 5 they actually select the $p=20$ lottery.
} 


\begin{tabular}{|c|c|c|c|}
\hline & & PROB & DET \\
\hline \multicolumn{2}{|c|}{ All Outcomes } & 26.6 & 52.5 \\
\hline \multirow{3}{*}{ Some Outcomes } & Large Gains & 12.8 & 6.0 \\
\hline & Large Losses & 13.3 & 3.8 \\
\hline & $p=v_{L}$ outcomes & 3.2 & 4.4 \\
\hline \multicolumn{2}{|c|}{ No Outcome } & 40.4 & 29.5 \\
\hline \multicolumn{2}{|c|}{ Mistake } & 3.7 & 3.8 \\
\hline & Sum & 100.0 & 100.0 \\
\hline
\end{tabular}

Table 8: Reported Outcomes in Part 3 Advice.

Notes: 188 participants in PROB and 183 in DET. There are four outcomes $(v, p)$ where $v, p \in\left\{v_{L}, v_{H}\right\}$. The first row shows all subjects who mention all four outcomes in any form. The bottom five rows contain subjects who report on specific subsets of outcomes: Large Gains: $\left\{\left(v_{L}, v_{L}\right)\right.$ and $\left.\left(v_{H}, v_{H}\right)\right\},\left\{\left(v_{H}, v_{H}\right)\right\},\left\{\left(v_{L}, v_{H}\right)\right.$ and explicitly $\left.\left(v_{H}, v_{H}\right)\right\}$, $\left\{\left(v_{L}, v_{L}\right),\left(v_{H}, v_{L}\right)\right.$ and $\left.\left(v_{H}, v_{H}\right)\right\}$. Large Losses: $\left\{\left(v_{L}, v_{H}\right)\right\},\left\{\left(v_{L}, v_{H}\right)\right.$ and implicitly $\left.\left(v_{H}, v_{H}\right)\right\},\left\{\left(v_{L}, v_{L}\right),\left(v_{H}, v_{L}\right)\right.$ and $\left.\left(v_{L}, v_{H}\right)\right\},\left\{\left(v_{L}, v_{L}\right),\left(v_{L}, v_{H}\right)\right.$ and $\left.\left(v_{H}, v_{H}\right)\right\} . p=v_{L}$ outcomes: $\left\{\left(v_{L}, v_{L}\right)\right\},\left\{\left(v_{L}, v_{L}\right)\right.$ and $\left.\left(v_{H}, v_{L}\right)\right\}$. No outcome: No outcome is reported in any form. Mistake: One of the payoffs is wrongly computed.

respectively). Furthermore, subjects who only mention large gains are quite often classified as Mix or $V_{H}$ in part 1: 62.5 and 54.6 percent in PROB and DET, respectively, compared to only 29.8 and 27.6 percent among the other subjects ( $p<0.01$ and $p=0.08$, respectively). However, subjects who only mention large gains and submit $p=v_{H}$ are probably not doing so because they are very risk seeking. Indeed, 62.5 and 90.9 percent of subjects who mention large gains in PROB and DET, respectively, select the safe alternative (corresponding to $p=v_{L}$ ) when in part 5 they face the lotteries that corresponds to the problem $\left(v_{L}, v_{H}\right)=(20,120)$.

Subjects who mention only large losses are very likely to recommend $p=v_{L}: 80.0$ and 85.7 percent in PROB and DET, respectively. This is larger than the 20.3 and 21.3 percent among subjects who do not mention only large losses - and neither all four outcomes ( $p<0.01$ and $p<0.01$, respectively). Furthermore, subjects who only mention large losses are quite often classified as $V_{L}$ in part 1: 40 and 100 percent in PROB and DET, respectively, compared to only 14.2 and 15.0 percent among the other subjects $(p<0.01$ and $p<0.01$, respectively). While we do not have sufficiently many subjects in DET for a detailed analysis, subjects in PROB who only mention large losses are more likely to be classified as a Focal type with $V_{L}$ in part 1 (such as $V_{L} V_{L}$ or $V_{L} M i x$ ), that is a type who is definitely not profit maximizing, 21.9 percent compared to only 5.7 percent among other subjects $(p<0.01)$.

To summarize, the results in this section point towards subjects in DET being much more likely to think of all four outcomes $(v, p)$ where $v, p \in\left\{v_{L}, v_{H}\right\}$. Subjects who mention all four outcomes in their advice are significantly more likely to be classified as $V_{L} V_{H}$. Furthermore, controlling for whether the advice mentions all four outcomes accounts for the differences between PROB and DET. It seems that the fact that the two companies exist in DET, rather than being possible states in PROB, makes it easier for subjects to think about the outcomes they receive for each firm for a given 


\begin{tabular}{|c|c|c|c|c|c|}
\hline & $\begin{array}{l}(1) \\
V_{L}^{4} \\
\end{array}$ & $\begin{array}{l}(2) \\
V_{H}^{4} \\
\end{array}$ & $\begin{array}{c}(3) \\
M i x^{4} \\
\end{array}$ & $\begin{array}{c}(4) \\
D^{4} m^{4} \\
\end{array}$ & $D_{o m}^{4}$ or $\operatorname{Res}^{4}$ \\
\hline Det & $\begin{array}{r}-0.103 \\
(0.063)\end{array}$ & $\begin{array}{c}0.045 \\
(0.056)\end{array}$ & $\begin{array}{c}0.084^{*} \\
(0.050)\end{array}$ & $\begin{array}{c}0.010 \\
(0.052)\end{array}$ & $\begin{array}{r}-0.026 \\
(0.059)\end{array}$ \\
\hline Num Risky & $\begin{array}{r}-0.059^{*} \\
(0.032)\end{array}$ & $\begin{array}{c}0.054^{*} \\
(0.029)\end{array}$ & $\begin{array}{c}-0.025 \\
(0.026)\end{array}$ & $\begin{array}{c}0.036 \\
(0.027)\end{array}$ & $\begin{array}{c}0.030 \\
(0.030)\end{array}$ \\
\hline Num Risky $\times$ Det & $\begin{array}{c}-0.061 \\
(0.047)\end{array}$ & $\begin{array}{c}0.016 \\
(0.042)\end{array}$ & $\begin{array}{c}0.038 \\
(0.037)\end{array}$ & $\begin{array}{c}-0.017 \\
(0.039)\end{array}$ & $\begin{array}{c}0.006 \\
(0.044)\end{array}$ \\
\hline Advice mentions all outcomes & $\begin{array}{l}0.134^{* *} \\
(0.057)\end{array}$ & $\begin{array}{l}-0.106^{* *} \\
(0.051)\end{array}$ & $\begin{array}{c}0.030 \\
(0.045)\end{array}$ & $\begin{array}{r}-0.089^{*} \\
(0.047)\end{array}$ & $\begin{array}{c}-0.057 \\
(0.053)\end{array}$ \\
\hline$V_{L} V_{H}$ & $\begin{array}{l}0.331^{* * *} \\
(0.059)\end{array}$ & $\begin{array}{r}-0.030 \\
(0.053)\end{array}$ & $\begin{array}{c}-0.049 \\
(0.047)\end{array}$ & $\begin{array}{l}-0.188^{* * *} \\
(0.049)\end{array}$ & $\begin{array}{l}-0.252^{* * *} \\
(0.055)\end{array}$ \\
\hline Constant & $\begin{array}{l}0.312^{* * *} \\
(0.074)\end{array}$ & $\begin{array}{l}0.210^{* * *} \\
(0.066)\end{array}$ & $\begin{array}{c}0.068 \\
(0.059)\end{array}$ & $\begin{array}{l}0.289^{* * *} \\
(0.061)\end{array}$ & $\begin{array}{l}0.409^{* * *} \\
(0.069)\end{array}$ \\
\hline Observations & 371 & 371 & 371 & 371 & 371 \\
\hline
\end{tabular}

Table 9: Main Treatments: Estimation output using part 4 types

Notes: Results from a linear regression. The dependent variable takes value 1 if the subject is classified as (1) $V_{L}^{4},(2) V_{H}^{4},(3)$ $M i x^{4},(4) D o m^{4}$, or (5) Dom ${ }^{4}$ or $R_{e s}^{4}$. Det is a treatment dummy that equals 1 if the subject participated in DET, which in this case means that those subjects are facing probabilistic problems. Num Risky is the number of risky lotteries the subject chooses in part 5 (from 0 to 3 ). $V_{L} V_{H}$ is a dummy that takes value 1 if the subject was classified as $V_{L} V_{H}$ in parts 1 and 2. Advice mentions all outcomes is a dummy that equals 1 if the advice of the subject mentions all four outcomes $(v, p)$ with $v, p \in\left\{v_{L}, v_{H}\right\}$. The regression also includes demographic controls (Gender, Ethnicity, Age and Schooling) and a control for the number of errors in the instructions. The full output is presented in Table 36 of the Online Appendix.

price. This, in turn, seems to be responsible for the Power of Certainty.

\subsection{Across Treatment Adaptation and Mentioning all outcomes}

We provide a second piece of evidence as to the relevance of thinking about all four outcomes, or more precisely, of subjects mentioning all four outcomes in the advice. In part 4 , subjects encounter 10 problems that have the same characteristics as those of part 1, including that $2 v_{L}<v_{H}$. Subjects now, however, play the opposite treatment than in part 1, that is, subjects in DET encounter probabilistic problems and subjects in PROB face deterministic problems in part 4. Note that part 4 comes after part 3, where subjects provided advice. So, instead of connecting their part 3 advice to the strategies that subjects were classified as before giving advice, we now use the advice as a control for their future part-4 behavior.

We classify subjects in part 4 based on the prices submitted in the last 5 rounds. Note that in the deterministic problems (encountered in part 4 of PROB), $p=v_{L}$ is the dominant strategy, while in probabilistic problems very risk-seeking subjects might find that $p=v_{H}$ maximizes their earnings.

All other prices are dominated. Using linear regressions we show that subjects who are classified as $V_{L} V_{H}$ in part 1 are significantly more likely to be classified as $V_{L}^{4}$ in part 4 (that is submitting $p=v_{L}$ in the last 5 rounds of part 4), and less likely to be classified as $D_{o m}^{4}$ (submitting dominated prices in part 4). Table 9 also shows that subjects who mention all four outcomes are more likely to be classified as $V_{L}^{4}$ and less likely to be classified as $D o m^{4}$ or $V_{H}^{4}$. 


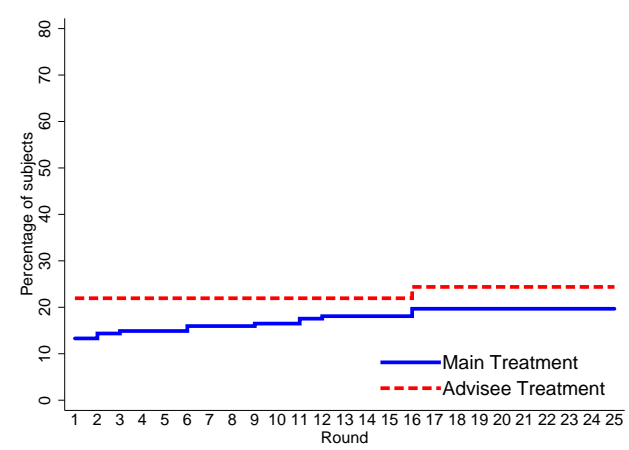

(a) Probabilistic: $v_{L}: n$ to 15 and $V_{L} V_{H}$.

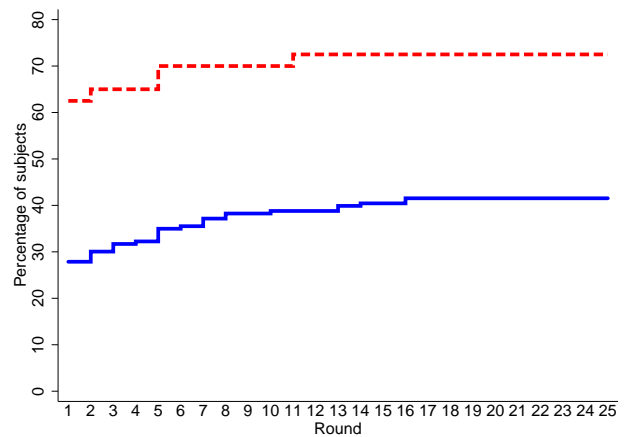

(b) Deterministic: $v_{L}: n$ to 15 and $V_{L} V_{H}$.

Figure 5: Evolution of Types in Main and Advisee Treatments [as \% of subjects].

The results from part 4 confirm that mentioning all four outcomes is also relevant in determining a subject's ability to submit optimal prices in an environment that is slightly different than the one they encountered before.

\subsection{Advisee Treatment}

We have two goals for this section: First, to incentivize the advice, we ran the Advisee treatments and want to report on whether advice can affect the behavior of subjects who receive advice. Second, we want to provide some insight as to the role of receiving and recognizing the usefulness of advice that mentions all four outcomes. Specifically, subjects in the Advisee treatment saw a piece of advice from five different subjects about what price to submit in round 1 of part 1, which corresponds to the problem $v_{L}=20$ and $v_{H}=120$. Subjects in ADVPROB (ADVDET) received advice from subjects in PROB (DET). The subjects then faced part 1 and part 2 from the main treatments.

We present two analyses of the advisee treatments. First, we compare how prices submitted by advisees compare to prices submitted by subjects in the main treatments who have not received any piece of advice. Second, we provide some evidence that subjects who receive and understand the usefulness of advice that mentions all four outcomes behave differently than other subjects.

Figure 5a shows the evolution of strategies that lead to $V_{L} V_{H}$ of subjects in ADVPROB - who received five pieces of advice - and of subjects in PROB - who received no advice, see Figure 8 in Appendix D for other types. There is almost no difference between these two groups of subjects. Table 10 shows the distribution of types (based on subject's choices in the last five rounds of part 1 and the five rounds of part 2). For instance, 19.7 percent of subjects are classified as type $V_{L} V_{H}$ in PROB (see Table 2), compared to 24.4 percent in ADVPROB. Table 11 confirms that there are no significant differences in type distributions between ADVPROB and PROB.

Figure 5b shows that there is a large difference in the evolution of strategies that lead to $V_{L} V_{H}$ between ADVDET and DET: For deterministic problems, a subject who received five pieces of advice from other subjects is almost twice as likely to submit $p=v_{L}$ from round $n$ to 15 and be classified as 


\begin{tabular}{ccccccc|c}
\hline \hline & & $V_{L} V_{H}$ & $\left\{\right.$ Mix $\left.V_{H}, V_{H} V_{H}\right\}$ & Focal & Dominated & Residual & Participants \\
\hline All & ADVProB & 24.4 & 22.0 & 22.0 & 14.6 & 17.1 & 41 \\
& ADVDET & 72.5 & 5.0 & 10.0 & 7.5 & 5.0 & 40 \\
\hline Selected Advice & ADVProB & 33.3 & 16.7 & 16.7 & 11.1 & 22.2 & 18 \\
that mentions all outcomes & ADVDET & 78.1 & 3.1 & 6.3 & 6.3 & 6.2 & 32 \\
Did not Select Advice & ADVPROB & 17.4 & 26.1 & 26.1 & 17.4 & 13.0 & 23 \\
that mentions all outcomes & ADVDET & 50.0 & 12.5 & 25.0 & 12.5 & 0.0 & 8 \\
\hline \hline
\end{tabular}

Table 10: Type Classification in Advisee Treatments [as \% of participants].

Notes: Type are defined based on the prices $p_{1}$ submitted in the last five rounds of part 1 and $p_{2}$ submitted in all five rounds of part 2. Type $V_{L} V_{H}: p_{1}=v_{L}$ and $p_{2}=v_{H}$. Type $\left\{M i x V_{H}, V_{H} V_{H}\right\}: p_{1} \in\left\{v_{L}, v_{H}\right\}$ and at least one $p_{1}=v_{H}$ and $p_{2}=v_{H}$. Type 'Focal': $p_{1}, p_{2} \in\left\{v_{L}, v_{H}\right\}$ and at least one $p_{2}=v_{L}$ (corresponds to $V_{L} V_{L}, V_{L} \operatorname{Mix}, \operatorname{Mix} V_{L}, \operatorname{MixMix}, V_{H} V_{L}$, or $V_{H}$ Mix. Type 'Dom': $p_{1}, p_{2} \notin\left\{v_{L}, v_{H}\right\}$. Residual: All remaining subjects.

\begin{tabular}{lccccc}
\hline & $(1)$ & $(2)$ & $(3)$ & $(4)$ & $(5)$ \\
& $V_{L} V_{H}$ & $\left\{M i x V_{H}, V_{H} V_{H}\right\}$ & Focal & Dom & Dom or Res \\
\hline PROB & & & & & \\
Advisee & 0.034 & -0.046 & 0.098 & -0.056 & -0.087 \\
& $(0.083)$ & $(0.090)$ & $(0.083)$ & $(0.083)$ & $(0.098)$ \\
Constant & $0.222^{* * *}$ & $0.148^{*}$ & $0.241^{* * *}$ & $0.256^{* * *}$ & $0.388^{* * *}$ \\
& $(0.076)$ & $(0.082)$ & $(0.076)$ & $(0.076)$ & $(0.089)$ \\
\hline Observations & 223 & 223 & 223 & 223 & 223 \\
\hline \multirow{2}{*}{ DET } & & & & & -0.125 \\
Advisee & $0.249^{* *}$ & $-0.136^{*}$ & 0.013 & -0.012 & $(0.088)$ \\
& $(0.100)$ & $(0.074)$ & $(0.060)$ & $(0.071)$ & 0.062 \\
Constant & $0.596^{* * *}$ & $0.203^{* * *}$ & $0.139^{* *}$ & 0.001 & $(0.082)$ \\
\hline Observations & $(0.093)$ & $(0.069)$ & $(0.056)$ & $(0.067)$ & 229 \\
\hline
\end{tabular}

Table 11: Main Treatment v. Advisee Treatment: Individual Types Estimations Output

Notes: Results from two linear regressions: PROB compares probabilistic treatments (Main and Advice), DET compares deterministic treatments (Main and Advice). The dependent variable takes value 1 if the subject is classified as (1) $V_{L} V_{H}$. (2) $M i x V_{H}$, $V_{H} V_{H}$ (3) Focal, (4) Dom, (5) Dom or Res. Advisee is a dummy variable that takes value 1 if the observation corresponds to the Advisee treatment. The regression also includes demographic controls (Gender, Ethnicity, Age and Schooling) and a control for the number of errors in the instructions. The full output is presented in Tables 29 and 30 of the Online Appendix. 


\begin{tabular}{|c|c|c|c|c|c|}
\hline & $\begin{array}{c}(1) \\
V_{L} V_{H} \\
\end{array}$ & $\left\{M i x V_{H}^{(2)}, V_{H} V_{H}\right\}$ & $\begin{array}{c}(3) \\
\text { Focal }\end{array}$ & $\begin{array}{c}(4) \\
\text { Dom }\end{array}$ & $\begin{array}{c}(5) \\
\text { Dom or Res }\end{array}$ \\
\hline Det & $\begin{array}{l}0.373^{* * *} \\
(0.104)\end{array}$ & $\begin{array}{c}-0.173^{* *} \\
(0.080)\end{array}$ & $\begin{array}{c}-0.058 \\
(0.089)\end{array}$ & $\begin{array}{c}-0.022 \\
(0.076)\end{array}$ & $\begin{array}{c}-0.141 \\
(0.092)\end{array}$ \\
\hline Selected Advice mentions all outcomes & $\begin{array}{c}0.253^{* *} \\
(0.107)\end{array}$ & $\begin{array}{c}-0.034 \\
(0.082)\end{array}$ & $\begin{array}{c}-0.146 \\
(0.092)\end{array}$ & $\begin{array}{c}-0.111 \\
(0.078)\end{array}$ & $\begin{array}{c}-0.074 \\
(0.095)\end{array}$ \\
\hline Constant & $\begin{array}{c}0.331^{* *} \\
(0.165)\end{array}$ & $\begin{array}{c}0.139 \\
(0.126)\end{array}$ & $\begin{array}{l}0.438^{* * *} \\
(0.141)\end{array}$ & $\begin{array}{c}0.158 \\
(0.121)\end{array}$ & $\begin{array}{c}0.091 \\
(0.147)\end{array}$ \\
\hline Observations & 81 & 81 & 81 & 81 & 81 \\
\hline
\end{tabular}

Table 12: Advisee Treatments: Individual Types Estimations Output in Parts 1 and 2

Notes: Results from a linear regression. The dependent variable takes value 1 if the subject is classified as (1) $V_{L} V_{H}$, (2) $\left\{\right.$ Mix $V_{H}$, $\left.V_{H} V_{H}\right\}$ (3) Focal, (4) Dom, (5) Dom or Res. Det is a dummy variable that takes value 1 if the observation corresponds to the deterministic treatment. The variable 'Selected Advice mentions all outcomes' is a dummy variable that takes value 1 if the advice selected as the 'most helpful' by the advisee mentions all four outcomes. The regression also includes demographic controls (Gender, Ethnicity, Age and Schooling) and a control for the number of errors in the instructions. The full output is presented in Table 31 of the Online Appendix.

$V_{L} V_{H}$ than a subject who received no advice, see Figure 8 in Appendix D for other types. Table 10 shows the distribution of types in ADVDET, where we have almost three quarter of all subjects being classified as the dominant type! The regressions in Table 11 confirm that in deterministic settings, subjects in the Advisee treatment are significantly more likely to be classified as the dominant strategy type and less likely to be classified as using the dominated strategy of $\left\{V_{H} V_{H}, M i x V_{H}\right\}$.

The fact that we have large improvements in terms of subjects classified as using optimal strategies when moving from subjects in DET to subjects in ADVDET is reminiscent of the common finding that decisions made with advice are closer to the predictions of economic theory than choices made without advice. ${ }^{46}$ However, this is not borne out in probabilistic problems where advice does not help subjects make better choices. This highlights that explaining and interpreting advice in PROB seems to be relatively difficult.

We now address whether receiving advice that mentions all four outcomes - and understanding that this is useful advice - is important. The bottom rows of Table 10 show the distribution of types for subjects who received an advice that mentions all four outcomes and selected one of those as the most helpful advice. The regression in Table 12 shows that subjects who selected an advice that mentions all four outcomes as the most helpful advice are more likely to be classified as $V_{L} V_{H}$ than those that did not. Independent of that, subjects in ADVDET are more likely to be classified as $V_{L} V_{H}$.

While subjects in ADVDET are more likely to receive an advice that mentions all four outcomes, recall that subjects received advice from five subjects, that is five pieces of advice. ${ }^{47}$ We can thus inquire whether when aggregating across all five pieces of advice, it is the case that subjects

\footnotetext{
${ }^{46}$ For details on choices with and without advice see the survey of Schotter (2003) and references therein.

${ }^{47}$ Table 27 in Appendix D shows the classification into types dependent on whether subjects received an advice that mentions all four outcomes, whether they selected it or not. All but one subject in ADVDET received an advice that mentioned all four outcomes. This is why we could not use the variable "received advice that mentions four outcomes" when accounting for type classification in the Advisee treatments.
} 
received information on all four outcomes. We find that 97.5 percent of subjects in ADVDET received information on all four outcomes across the five pieces of advice, which is not very different from the 95.1 percent of subjects in ADVPROB. ${ }^{48}$

The fact that subjects who received advice that mentions all four outcomes and recognized its significance are more likely to be classified as $V_{L} V_{H}$ provides some confirmation that thinking about the four possible outcomes $(v, p)$ with $v, p \in\left\{v_{L}, v_{H}\right\}$ is crucial to be able to behave optimally in the acquiring a company problems.

\subsection{Focusing on Certainty: One-Firm treatments}

\begin{tabular}{ccccccc|c}
\hline \hline & & $V_{L} V_{H}$ & $\left\{\right.$ Mix $\left.V_{H}, V_{H} V_{H}\right\}$ & Focal & Dominated & Residual & Participants \\
\hline \multirow{2}{*}{ All } & ONEFIRM $_{\mathrm{PROB}}$ & 27.8 & 25.5 & 22.2 & 12.5 & 12.0 & 212 \\
& ONEFIRM $_{\text {DET }}$ & 47.2 & 18.4 & 8.0 & 9.9 & 16.5 & 216 \\
\hline$V$ & ONEFIRM $_{\text {PROB }}$ & 41.7 & 28.1 & 27.3 & 0.0 & 2.9 & 139 \\
& ONEFIRM $_{\text {DET }}$ & 65.1 & 21.7 & 10.9 & 0.0 & 2.3 & 129 \\
\hline$V$ and & ONEFIRM $_{\mathrm{PROB}}$ & 51.1 & 17.0 & 28.7 & 0.0 & 3.2 & 94 \\
No risky lottery & ONEFIRM $_{\mathrm{DET}}$ & 72.0 & 17.2 & 10.8 & 0.0 & 0.0 & 93 \\
\hline \hline
\end{tabular}

Table 13: ONEFIRM Treatments: Parts 3 and 4 Type Classification [as \% of participants]

Notes: Types are defined based on the prices $p_{1}$ submitted in the last five rounds of part 3 and $p_{2}$ submitted in all five rounds of part 4. Type $V_{L} V_{H}: p_{1}=v_{L}$ and $p_{2}=v_{H}$. Type $\left\{M i x V_{H}, V_{H} V_{H}\right\}: p_{1} \in\left\{v_{L}, v_{H}\right\}$ and at least one $p_{1}=v_{H}$ and $p_{2}=v_{H}$. Type 'Focal': $p_{1}, p_{2} \in\left\{v_{L}, v_{H}\right\}$ and at least one $p_{2}=v_{L}$ (corresponds to $V_{L} V_{L}, V_{L}$ Mix, Mix $V_{L}, \operatorname{MixMix}, V_{H} V_{L}$, or $V_{H}$ Mix). Type 'Dom': $p_{1}, p_{2} \notin\left\{v_{L}, v_{H}\right\}$. Residual: All remaining subjects. In onefirm subjects are classified as $V$ if they submit $p=v$ in rounds 16-25 (last 5 rounds of part 1 and all rounds of part 2).

We have seen that subjects who think of all four outcomes $(v, p)$ with $v, p \in\left\{v_{L}, v_{H}\right\}$ are more likely to be classified as using a profit-maximizing strategy. In the One-Firm treatments subjects are first confronted with 25 rounds of a simplification of part 1 and part 2 of the main treatments. Specifically, when submitting a price, subjects do so only for a single firm of which they know the value. This may increase the chance that subjects focus on outcomes they receive when submitting a price. We therefore ask whether this helps to increase the fraction of profit-maximizing strategies and reduce or even eliminate the role of $\mathrm{PoC}$, that is eliminate differences between probabilistic and deterministic problems. Specifically, in part 3, part 4 and part 5 of the ONEFIRM treatments subjects face part 1, part 2 and part 5 of either PROB or DET, we refer to the treatments as ONEFIRM ${ }_{\mathrm{PROB}}$ and ONEFIRM $_{\text {DET }}$, respectively. Therefore, in the ONEFIRM treatments, subjects are trained for 25 periods to think about the value of the company when submitting a price before they encounter part 1 and 2 of the main treatments.

\footnotetext{
${ }^{48} \mathrm{~A}$ linear regression with a treatment dummy on the right-hand side indicates that the treatment effect is small (0.024) and not statistically significant (p-value 0.577).
} 


\begin{tabular}{|c|c|c|c|c|c|}
\hline & $\begin{array}{c}(1) \\
V_{L} V_{H}\end{array}$ & $\begin{array}{c}(2) \\
\left\{M i x V_{H}, V_{H} V_{H}\right\}\end{array}$ & $\begin{array}{c}(3) \\
\text { Focal }\end{array}$ & $\begin{array}{c}(4) \\
\text { Dom }\end{array}$ & $\begin{array}{c}(5) \\
\text { Dom or Res }\end{array}$ \\
\hline \multicolumn{6}{|l|}{ PROB } \\
\hline One Firm & $\begin{array}{l}0.123^{* *} \\
(0.053)\end{array}$ & $\begin{array}{c}-0.064 \\
(0.057)\end{array}$ & $\begin{array}{c}0.084 \\
(0.054)\end{array}$ & $\begin{array}{r}-0.092^{*} \\
(0.047)\end{array}$ & $\begin{array}{l}-0.142^{* *} \\
(0.057)\end{array}$ \\
\hline Num Risky & $\begin{array}{r}-0.057^{*} \\
(0.029)\end{array}$ & $\begin{array}{c}-0.008 \\
(0.031)\end{array}$ & $\begin{array}{c}-0.008 \\
(0.029)\end{array}$ & $\begin{array}{l}0.071^{* * *} \\
(0.026)\end{array}$ & $\begin{array}{l}0.073^{* *} \\
(0.031)\end{array}$ \\
\hline Num Risky $\times$ One Firm & $\begin{array}{c}-0.062 \\
(0.041)\end{array}$ & $\begin{array}{l}0.109^{* *} \\
(0.044)\end{array}$ & $\begin{array}{r}-0.013 \\
(0.041)\end{array}$ & $\begin{array}{r}-0.063^{*} \\
(0.036)\end{array}$ & $\begin{array}{c}-0.034 \\
(0.044)\end{array}$ \\
\hline Constant & $\begin{array}{l}0.339^{* * *} \\
(0.062)\end{array}$ & $\begin{array}{l}0.257^{* * *} \\
(0.066)\end{array}$ & $\begin{array}{l}0.156^{* *} \\
(0.062)\end{array}$ & $\begin{array}{l}0.146^{* * *} \\
(0.054)\end{array}$ & $\begin{array}{l}0.249^{* * *} \\
(0.066)\end{array}$ \\
\hline Observations & 404 & 404 & 404 & 404 & 404 \\
\hline \multicolumn{6}{|l|}{ DET } \\
\hline One Firm & $\begin{array}{c}0.091 \\
(0.067)\end{array}$ & $\begin{array}{c}-0.018 \\
(0.053)\end{array}$ & $\begin{array}{r}-0.026 \\
(0.039)\end{array}$ & $\begin{array}{c}-0.040 \\
(0.045)\end{array}$ & $\begin{array}{c}-0.047 \\
(0.060)\end{array}$ \\
\hline Num Risky & $\begin{array}{c}-0.017 \\
(0.037)\end{array}$ & $\begin{array}{c}0.033 \\
(0.029)\end{array}$ & $\begin{array}{r}-0.015 \\
(0.022)\end{array}$ & $\begin{array}{c}0.020 \\
(0.025)\end{array}$ & $\begin{array}{c}-0.002 \\
(0.033)\end{array}$ \\
\hline Num Risky $\times$ One Firm & $\begin{array}{c}-0.077 \\
(0.048)\end{array}$ & $\begin{array}{c}-0.018 \\
(0.038)\end{array}$ & $\begin{array}{c}0.015 \\
(0.028)\end{array}$ & $\begin{array}{c}-0.001 \\
(0.032)\end{array}$ & $\begin{array}{r}0.080^{*} \\
(0.043)\end{array}$ \\
\hline Constant & $\begin{array}{l}0.518^{* * *} \\
(0.077)\end{array}$ & $\begin{array}{l}0.218^{* * *} \\
(0.061)\end{array}$ & $\begin{array}{l}0.126^{* * *} \\
(0.045)\end{array}$ & $\begin{array}{c}0.049 \\
(0.052)\end{array}$ & $\begin{array}{l}0.138^{* *} \\
(0.070)\end{array}$ \\
\hline Observations & 395 & 395 & 395 & 395 & 395 \\
\hline
\end{tabular}

Table 14: Estimation Output: One Firm Treatments (Parts 3 and 4) compared to Main Treatments (Parts 1 and 2)

Notes: Summarizes results from two linear regressions: РRов compares probabilistic treatments (Main and One Firm), DET compares deterministic treatments (Main and One Firm). The dependent variable takes value 1 if the subject is classified as (1) $V_{L} V_{H}$, (2) $\left\{M i x V_{H}, V_{H} V_{H}\right\}$ (3) Focal, (4) Dom, (5) Dom or Res. One Firm=1 is a dummy variable that takes value 1 if the observation corresponds to the deterministic treatment. Num Risky is the number of risky lotteries the subject chooses in part 5 (from 0 to 3). The regression also includes demographic controls (Gender, Ethnicity, Age and Schooling) and a control for the number of errors in the instructions. The full output is presented in Tables 32 and 33 of the Online Appendix.

Table 13 shows the distribution of types in the ONEFIRM treatments. The linear regression in Table 14 shows that in probabilistic problems, focusing first on one firm significantly increases the chance with which subjects are classified as the dominant type $V_{L} V_{H}$ and decreases the chance to be classified as the Dominated type or being unclassified (Residual type). There is no such comparable change in the deterministic problems.

It seems that focusing one one firm of known value helps subjects to subsequently only submit prices $p \in\left\{v_{L}, v_{H}\right\}$. However, Table 13 shows that there is still a large difference between the probabilistic and deterministic problems. Indeed, the linear regression in Table 15 shows that subjects in ONEFIRM $_{\text {DET }}$ are significantly more likely to be classified as $V_{L} V_{H}$ than subjects in ONEFIRM ${ }_{\mathrm{PROB}}$, and less likely to be classified as using a Focal (and hence dominated) strategy.

The coefficients on $V$ in regressions (4) and (5) of Table 15, where $V$ is a dummy controlling for whether the subject submitted $p=v$ in the last 10 rounds of parts 1 and 2 in which subjects submit prices for one firm of known value, show that being able to solve the one firm problem significantly 
reduces instances of prices $p \notin\left\{v_{L}, v_{H}\right\} .{ }^{49}$ However, subjects classified as $V$ are not only more likely to be classified as $V_{L} V_{H}$, but also as using Focal strategies, which are strategies that are dominated (though only using prices $p \in\left\{v_{L}, v_{H}\right\}$ ).

Finally, we use subjects in the ONEFIRM treatments to reassess the role of $P o C$ on the general problem of Computational Complexity. Table 13 shows that 27.8 percent of subjects are classified as $V_{L} V_{H}$ in parts 3 and 4 in ONEFIRM ${ }_{\mathrm{PROB}}$, compared to 47.2 percent in ONEFIRM DET $_{\text {. Overall, } 62.6}$ percent of subjects are classified as $V$ in parts 1 and 2 in the ONEFIRM treatments. So, using only $V_{L} V_{H}$ as the dominant strategy, $P o C$ accounts for 55.7 percent of what conventionally was labelled as problems of complexity.

\begin{tabular}{lccccc}
\hline & $(1)$ & $(2)$ & $(3)$ & $(4)$ & $(5)$ \\
& $V_{L} V_{H}$ & $\left\{M i x V_{H}, V_{H} V_{H}\right\}$ & Focal & Dom & Dom or Res \\
\hline Det & $0.255^{* * *}$ & -0.045 & $-0.178^{* * *}$ & -0.048 & -0.032 \\
& $(0.055)$ & $(0.054)$ & $(0.047)$ & $(0.036)$ & $(0.041)$ \\
Num Risky & $-0.091^{* * *}$ & $0.102^{* * *}$ & -0.015 & -0.007 & 0.004 \\
& $(0.029)$ & $(0.029)$ & $(0.025)$ & $(0.019)$ & $(0.022)$ \\
Num Risky $\times$ Det & 0.018 & $-0.082^{* *}$ & 0.023 & 0.012 & 0.041 \\
& $(0.040)$ & $(0.039)$ & $(0.034)$ & $(0.026)$ & $(0.030)$ \\
$V$ & $0.367^{* * *}$ & $0.103^{* *}$ & $0.104^{* * *}$ & $-0.258^{* * *}$ & $-0.574^{* * *}$ \\
& $(0.044)$ & $(0.043)$ & $(0.037)$ & $(0.029)$ & $(0.033)$ \\
Constant & $0.179^{* * *}$ & $0.191^{* * *}$ & $0.144^{* *}$ & $0.223^{* * *}$ & $0.487^{* * *}$ \\
& $(0.067)$ & $(0.066)$ & $(0.057)$ & $(0.044)$ & $(0.051)$ \\
\hline Observations & 428 & 428 & 428 & 428 & 428 \\
\hline
\end{tabular}

Table 15: ONEFIRM Treatments: Estimation output using last 5 rounds of part 3 and part 4 for the classification of types.

Notes: Results from a linear regression. The dependent variable takes value 1 if the subject is classified as (1) $V_{L} V_{H},(2)$ $\left\{M i x V_{H}, V_{H} V_{H}\right\}$ (3) Focal, (4) Dom, (5) Dom or Res. Det=1 is a dummy variable that takes value 1 if the observation corresponds to ONEFIRM ${ }_{\text {DET }}$ and 0 if it corresponds to ONEFIRM chooses in part 5 (from 0 to 3 ). The variable $V$ is a dummy variable that equals 1 if the subject selected $p=v$ in the last 10 periods of problems with one firm (last 5 periods of part 1 and 5 periods of part 2). The regression also includes demographic controls (Gender, Ethnicity, Age and Schooling) and a control for the number of errors in the instructions. The full output is presented in Table 34 of the Online Appendix.

Next we restrict attention to subjects who in parts 1 and 2 were classified as $V$ and who never took a risky lottery in part 5 , thereby guaranteeing that we restrict attention to subjects who really understand the one-firm problem and are not very risk seeking. Among those subjects, by definition, 100 percent are classified as $V$, though only 51.1 percent of them use $V_{L} V_{H}$ in ONEFIRM ProB $_{\text {, }}$, which should now comprise almost all subjects who use payoff-maximizing choices. Once more, the Power of Certainty accounts for 42.7 percent of the problem previously attributed to computational Complexity.

\footnotetext{
${ }^{49}$ This finding is consistent with the effect of experience reported by Charness and Levin (2009).
} 


\section{Discussion and Conclusions}

\subsection{Discussion and Additional Related Literature}

While most of the recent theoretical literature on biases in decision making cannot directly account for our results (e.g. Sims, 2003, Bordalo, Gennaioli and Shleifer, 2012, Gabaix, 2014, and Caplin, Dean and Leahy, 2016), there is one paper which does make predictions that differ between the probabilistic and the deterministic problems. Li (2017) can account for the main comparative static result in submitted strategies, though we conjecture perhaps not exactly for the right reasons. The paper introduces the concept of obvious strategy proofness to understand which strategy proof mechanisms are obviously so, that is, agents would actually recognize this fact and hence submit the dominant strategy. Specifically, a dominant strategy is defined as obviously dominant if the best outcome from a deviation is no better than the worst outcome from following the dominant strategy.

In contrast to the motivating environments in Li (2017), we have no strategic interaction but the concept of obvious strategy proofness can still be applied. Submitting $p=v_{L}$ is a dominant and indeed obviously-dominant strategy in part 1 of DET (where $2 v_{L}<v_{H}$ ), since the return from $p=v_{L}$ is $0.5 v_{L}$, while that from $p=v_{H}$ is $1.5 v_{L}-0.5 v_{H}<0.5 v_{L}$. However, $p=v_{L}$ is not an obviouslydominant strategy in PROB: the best outcome from $p=v_{H}$ is $0.5 v_{H}$, which is larger than the worst outcome from $p=v_{L}$, which is 0 . Similarly, for part 2, it is possible to show that $p=v_{H}$ is obviously dominant only in DET but not in PROB. If agents are able to recognize dominant strategies only if these are obviously dominant, then we would expect a comparative static between PROB and DET such as the one observed in the paper.

One reason we believe that obvious dominance may not be exactly the right concept in our environment is that we were able to link the failure to play the dominant strategy to the failure to mention all four outcomes $(v, p)$, where $v, p \in\left\{v_{L}, v_{H}\right\}$. Furthermore, it is not straightforward to link an agent who can only compare sets, as in the best outcome from deviation to the worst outcome using the dominant strategy, to an agent who seems to actually not think about all possible outcomes. One question open for future research is to design an experimental test that assesses if certainty helps even if profit-maximizing strategies are obviously dominant.

Another strand of the literature studies contingent thinking using Savage's sure-thing principle (Savage, 1972). The sure-thing principle asserts that if a person prefers $A$ to $B$ knowing that event $X$ obtains and also prefers $A$ to $B$ knowing that $X$ does not obtain, then the person prefers $A$ to $B$ prior to learning whether $X$ results or not. There is literature, however, showing that individuals often violate the sure-thing principle, a phenomenon referred to as the disjunction effect (see Shafir and Tversky, 1992, Tversky and Shafir, 1992, Shafir, 1994, Croson and Esponda and Vespa, 2017a). ${ }^{50}$

\footnotetext{
${ }^{50}$ The psychology model for the disjunction effect is that individuals do not make choices based on consequences of decisions, but rather based on reasons for making one choice over another. For example, suppose a student would want to take a vacation in Hawaii when they pass a big test to celebrate and when they fail this big test then in order to console themselves. Then, if the student has to decide whether to go to Hawaii before knowing the test results, the student has no reason to go, violating the sure thing principle, see Shafir, Simonson and Tversky (1993).
} 
In particular, Esponda and Vespa (2017a) find that failure to satisfy the sure-thing principle can be related to difficulties in thinking through the state-space. Notice that our experiment does not test the sure-thing principle because depending on the state of the world, the firm being of low or high value, the individual would want to submit a different price. However, our findings do suggest a new mechanism for the violation of the sure-thing principle: Perhaps individuals cannot think about the different states of the world, and hence fail to envision the returns from various choices.

In this section we also want to discuss some possible alternative designs. Since the main goal of our paper was to show the $P_{o} C$, we selected two treatments that truly differ based on that hypothesis. PROB and DET are different from each other, and do not just have a different framing. There are, however, treatments where the difference to PROB may be more a difference of framing, though in which we would expect some of the $P o C$ to play a role. For example, consider the following variation, which we call the 'Late Lottery' treatment. The treatment is almost like DET, though, after having purchased none, one or two firms, we use a randomization device to decide which of the two possible transactions is actually used for payments. There is an equal chance that either the firm of value $v_{L}$ or the firm of value $v_{H}$ is chosen. This treatment is a re-framing of PROB. It may, however, nonetheless change behavior in a way that this 'Late Lottery' treatment is in between PROB and DET. One possible reason could be narrow bracketing, as at the time individuals make choices, the subject could ignore the final lottery draw, which makes the 'Late Lottery' treatment look like DET.

\subsection{Summary of Results and Conclusion}

We provide a new hypothesis, the Power of Certainty, to account for a significant portion of the difficulties agents experience when they have to engage in contingent reasoning. We propose that it is not just the fact that agents have to aggregate payoff-relevant information over multiple states that causes problems, but the fact that the states are uncertain. Specifically, in the 'Acquiring-aCompany' problem, an agent purchases a firm of value $v$ whenever $p \geq v$, in which case her profits for that firm are $1.5 v-p$. The case in which the agent has to submit a price before knowing whether the value of the firm is low $\left(v_{L}\right)$ or high $\left(v_{H}\right)$, each being equally likely, is notoriously difficult relative to the environment in which there is one firm of known value. These difficulties have been attributed to what is perhaps best described as computational complexities, arising from the fact that two values are more difficult to consider than a single value. We construct a new problem, the deterministic problem, in which there are two firms, one of value $v_{L}$ and one of value $v_{H}$. The agent submits a price, which is then sent to each firm separately. This ensures that for each price $p$, twice the expected payoffs in the probabilistic problem equal the realized payoffs in the deterministic problem. The deterministic problem retains the complexity of two firms but eliminates any uncertainty.

We ran experiments on Amazon Turk to compare subjects' price choices in a probabilistic and a deterministic treatment, which contained probabilistic problems (where payoffs are multiplied by two) and deterministic problems. In part 1 , where $2 v_{L}<v_{H}$, such as $v_{L}=20$ and $v_{H}=120$, for

subjects in the probabilistic treatment who are not very risk seeking, and for all subjects in the 
deterministic treatment, the dominant action is to submit a price $p=v_{L}$. In part 2 the dominant price for all subjects is to submit $p=v_{H}$ since $1.5 v_{L}>v_{H}$, such as $v_{L}=50$ and $v_{H}=60$. By varying the environment in a way that the dominant action changes, we ensure that subjects who are classified as payoff maximizing are not using rules of thumb in which the submitted price is independent of the actual values of the firms.

When we only consider subjects who both submit $p=v_{L}$ in part 1 and $p=v_{H}$ in part 2 as payoff maximizing, we find that there are twice as many such subjects in the deterministic than in the probabilistic treatment. Furthermore, we show that this difference cannot be accounted for by many subjects being risk loving (since risk-loving subjects in the probabilistic treatment may have different dominant strategies).

Finally, we show that the Power of Certainty is not only significant, but also accounts for a quantitatively large portion of the welfare loss subjects incur when moving from a problem involving one firm of known value to a problem where the firm could have two possible values, each realized with fifty percent chance. That is, we show that the Power of Certainty is responsible for a substantial part of the welfare loss that used to be attributed to computational complexity.

In the last part of the paper we provide some evidence for the causes of the Power of Certainty, that is why probabilistic problems of one firm with two possible values are harder than deterministic problems with two firms of known value. Using incentivized advice that subjects provide to a different participant for the $v_{L}=20$ and $v_{H}=120$ problem, we find that subjects in the deterministic treatment are twice as likely to mention the four outcomes that are needed to compute payoffmaximizing actions: For each price $p=v_{L}=20$ and $p=v_{H}=120$ the outcome that would result if the firm were of either low or high value. We show that this feature of the advice largely accounts for differences between the probabilistic and deterministic treatments. We therefore have evidence that eliminating uncertainty increases the subjects' tendency to mention all outcomes necessary to compute payoff-maximizing prices, and hence, presumably, to think about them in the first place.

We expect that the Power of Certainty hypothesis can help explain behavior in other settings. For example, it has some implications in trying to understand why we might often attribute firms to be more likely to be profit maximizers than consumers. While a standard argument is the competition among firms, we provide a new explanation: The Power of Certainty hypothesis suggests that any individual is much more capable in finding profit-maximizing strategies when playing against a distribution, than when playing a lottery from that distribution. Since firms may make many choices in similar environments compared to consumers, Power of Certainty asserts that even individuals deciding in the role of a firm would be better at profit maximization. There could be a whole range of environments in which the Power of Certainty could improve subjects' decision making, such choices of insurance, annuities, etc. We also suspect that $P o C$ may help agents in strategic settings such as auctions. Finally, we leave it to future research to provide a model that can account for the Power of Certainty. 


\section{Bibliography}

Ambuehl, Sandro, B. Douglas Bernheim and Annamaria Lusardi. 2014. "The effect of financial education on the quality of decision making." National Bureau of Economic Research .

Ambuehl, Sandro and Shengwu Li. 2017. "Belief updating and the demand for information." Games and Economic Behavior (forthcoming).

Araujo, Felipe, Stephanie Wang and Alistair J. Wilson. 2017. "Cursed through Time: Dynamic Adverse Selection in the Laboratory." Working paper .

Barrett, Garry F and Stephen G Donald. 2003. "Consistent tests for stochastic dominance." Econometrica 71(1):71-104.

Bazerman, Max H. and William F. Samuelson. 1983. "I won the auction but don't want the prize." Journal of Conflict Resolution 27(4):618-634.

Bénabou, Roland and Jean Tirole. 2002. "Self-confidence and personal motivation." The Quarterly Journal of Economics 117(3):871-915.

Bereby-Meyer, Yoella and Alvin E Roth. 2006. "The speed of learning in noisy games: Partial reinforcement and the sustainability of cooperation." The American Economic Review 96(4):10291042 .

Bordalo, Pedro, Nicola Gennaioli and Andrei Shleifer. 2012. "Salience theory of choice under risk." The Quarterly Journal of Economics 127(3):1243-1285.

Brunnermeier, Markus K and Jonathan A Parker. 2005. "Optimal expectations." The American Economic Review 95(4):1092-1118.

Caplin, Andrew and John Leahy. 2001. "Psychological expected utility theory and anticipatory feelings." The Quarterly Journal of Economics 116(1):55-79.

Caplin, Andrew and Mark Dean. 2015. "Revealed preference, rational inattention, and costly information acquisition." The American Economic Review 105(7):2183-2203.

Caplin, Andrew, Mark Dean and John Leahy. 2016. "Rational inattention, optimal consideration sets and stochastic choice." Working paper.

Charness, Gary and Dan Levin. 2009. "The origin of the winner's curse: a laboratory study." American Economic Journal: Microeconomics 1(1):207-236.

Costa-Gomes, Miguel A. and Georg Weizsäcker. 2008. "Stated beliefs and play in normal-form games." The Review of Economic Studies 75(3):729-762.

Costa-Gomes, Miguel and Vincent P Crawford. 2006. "Cognition and behavior in two-person guessing games: An experimental study." The American Economic Review 96(5):1737-1768. 
Crawford, Vincent P, Miguel A Costa-Gomes and Nagore Iriberri. 2013. "Structural models of nonequilibrium strategic thinking: Theory, evidence, and applications." Journal of Economic Literature 51(1):5-62.

Crawford, Vincent P. and Nagore Iriberri. 2007. "Level-k Auctions: Can a Nonequilibrium Model of Strategic Thinking Explain the Winner's Curse and Overbidding in Private-Value Auctions?" Econometrica 75(6):1721-1770.

Croson, Rachel TA. 1999. "The disjunction effect and reason-based choice in games." Organizational Behavior and Human Decision Processes 80(2):118-133.

Enke, Benjamin. 2017. "What You See Is All There Is." Working paper.

Enke, Benjamin and Florian Zimmermann. 2017. "Correlation neglect in belief formation." Working paper.

Esponda, Ignacio. 2008. "Behavioral equilibrium in economies with adverse selection." The American Economic Review 98(4):1269-1291.

Esponda, Ignacio and Emanuel Vespa. 2014. "Hypothetical Thinking and Information Extraction in the Laboratory." American Economic Journal: Microeconomics 6(4):180-202.

Esponda, Ignacio and Emanuel Vespa. 2017a. "Contingent Preferences and the Sure-Thing Principle: Revisiting Classic Anomalies in the Laboratory.".

Esponda, Ignacio and Emanuel Vespa. 2017b. "Endogenous sample selection: A laboratory study." Quantitative Economics (forthcoming).

Eyster, Erik and Georg Weizsäcker. 2010. "Correlation neglect in financial decision-making." Working Paper.

Eyster, Erik and Matthew Rabin. 2005. "Cursed equilibrium." Econometrica 73(5):1623-1672.

Eyster, Erik, Matthew Rabin and Georg Weizsäcker. 2015. "An Experiment on Social Mislearning." Working paper.

Fischbein, Efraim and Ditza Schnarch. 1997. "The evolution with age of probabilistic, intuitively based misconceptions." Journal for research in mathematics education pp. 96-105.

Fischhoff, Baruch. 1975. "Hindsight $\neq$ foresight: the effect of outcome knowledge on judgment under uncertainty." Journal of Experimental Psychology: Human Perception and Performance 1:288-299.

Fragiadakis, Daniel, Daniel Knoepfle and Muriel Niederle. 2017. "Who is Strategic?".

Gabaix, Xavier. 2014. "A sparsity-based model of bounded rationality." The Quarterly Journal of Economics 129(4):1661-1710. 
Gennaioli, Nicola and Andrei Shleifer. 2010. "What comes to mind." The Quarterly Journal of Economics 125(4):1399-1433.

Hamilton, William. 1878. Lectures on metaphysics. Sheldon.

Hoffrage, Ulrich, Gerd Gigerenzer, Stefan Krauss and Laura Martignon. 2002. "Representation facilitates reasoning: what natural frequencies are and what they are not." Cognition 84(3):343352.

Holt, Charles A and Susan K Laury. 2002. "Risk aversion and incentive effects." American Economic Review 92(5):1644-1655.

Ivanov, Asen, Dan Levin and Muriel Niederle. 2010. "Can relaxation of beliefs rationalize the winner's curse?: an experimental study." Econometrica 78(4):1435-1452.

Jehiel, Philippe. 2005. "Analogy-based expectation equilibrium." Journal of Economic Theory 123(2):81-104.

Kagel, John H. and Dan Levin. 1986. "The winner's curse and public information in common value auctions." The American Economic Review pp. 894-920.

Kahneman, Daniel and Amos Tversky. 1972. "Subjective probability: A judgment of representativeness." Cognitive Psychology 3(3):430-454.

Kahneman, Daniel and Amos Tversky. 1979. "Prospect theory: An analysis of decision under risk." Econometrica pp. 263-291.

Köszegi, Botond and Adam Szeidl. 2012. "A model of focusing in economic choice." The Quarterly Journal of Economics 128(1):53-104.

Laibson, David. 1997. "Golden eggs and hyperbolic discounting." The Quarterly Journal of Economics 112(2):443-478.

Langrall, Cynthia W and Edward S Mooney. 2005. "Characteristics of elementary school students' probabilistic reasoning." pp. 95-119.

Lennie, Peter. 2003. "The cost of cortical computation." Current Biology 13(6):493-497.

Levin, Dan, James Peck and Asen Ivanov. 2016. "Separating Bayesian updating from nonprobabilistic reasoning: An experimental investigation." American Economic Journal: Microeconomics 8(2):39-60.

Li, Shengwu. 2017. "Obviously strategy-proof mechanisms." American Economic Review (forthcoming) .

Mobius, Markus M, Muriel Niederle, Paul Niehaus and Tanya S Rosenblat. 2014. "Managing selfconfidence: Theory and experimental evidence." Working Paper . 
Nagel, Rosemarie. 1995. "Unraveling in guessing games: An experimental study." The American Economic Review 85(5):1313-1326.

Ngangoué, Kathleen and Georg Weizsäcker. 2017. "Learning from unrealized versus realized prices.".

Niederle, Muriel. 2016. "Gender." in The Handbook of Experimental Economics, Volume 2, edited by John H Kagel and Alvin E Roth. .

O’Donoghue, Ted and Matthew Rabin. 1999. "Doing it now or later." American Economic Review pp. 103-124.

Rabin, Matthew. 2000. "Risk aversion and expected-utility theory: A calibration theorem." Econometrica 68(5):1281-1292.

Rabin, Matthew and Joel L Schrag. 1999. "First impressions matter: A model of confirmatory bias." The Quarterly Journal of Economics 114(1):37-82.

Savage, Leonard J. 1972. The foundations of statistics. Courier Corporation.

Schotter, Andrew. 2003. "Decision making with naïve advice." American Economic Review pp. 196201.

Shafir, Eldar. 1994. "Uncertainty and the difficulty of thinking through disjunctions." Cognition $50(1): 403-430$.

Shafir, Eldar and Amos Tversky. 1992. "Thinking through uncertainty: Nonconsequential reasoning and choice." Cognitive Psychology 24(4):449-474.

Shafir, Eldar, Itamar Simonson and Amos Tversky. 1993. "Reason-based choice." Cognition 49(1):1136 .

Sims, Christopher A. 2003. "Implications of rational inattention." Journal of Monetary Economics 50(3):665-690.

Thompson, Suzanne C, Wade Armstrong and Craig Thomas. 1998. "Illusions of control, underestimations, and accuracy: a control heuristic explanation." Psychological Bulletin 123(2):143.

Tversky, Amos and Daniel Kahneman. 1974. "Judgment under Uncertainty: Heuristics and Biases." Science 185(4157):1124-1131.

Tversky, Amos and Eldar Shafir. 1992. "The disjunction effect in choice under uncertainty." Psychological Science 3(5):305-310.

Vespa, Emanuel and Alistair J. Wilson. 2016. "Communication with multiple senders: An experiment." Quantitative Economics 7(1):1-36. 


\section{A Main Findings Using Submitted Prices}

In the main part of the paper we analyze results and provide evidence for the Power of Complexity by classifying subjects into types. In this appendix we show that we reach similar conclusions if we use the aggregate distribution of submitted prices in part 1.

Most of our analysis in this section groups prices into five categories: $p<v_{L}, p=v_{L}, p \in\left(v_{L}, v_{H}\right)$, $p=v_{H}$ and $p>v_{H}$. However, we first provide a more detailed classification in Table 16, which further disaggregates two categories: $p \in\left(v_{L}, v_{H}\right)$ and $p>v_{H}$, to evaluate whether some prices are especially common. For example, we observe that $0.48(0.41)$ percent of prices submitted in PROB (DET) are the average of $v_{L}$ and $v_{H}\left(p=\frac{v_{L}+v_{H}}{2}\right)$. We also observe that some prices are equal to 1.5 times one of the values. This is the case for 1.36 and 0.38 percent of prices if $v=v_{L}$ for PROB and DET, respectively. We do not, however, observe a large mass of prices at any particular price $p \in\left(v_{L}, v_{H}\right) \cup\left(v_{H}, 150\right]$. The most common category is prices that are one unit above the value of the company, which is the focus of one of our robustness checks for the classification of types in the next section of this appendix.

\begin{tabular}{ccccc}
\hline \hline & \multicolumn{2}{c}{ PROB } & \multicolumn{2}{c}{ DET } \\
& All Rounds & Last 10 & All Rounds & Last 10 \\
\hline$p<v_{L}$ & 1.12 & 0.85 & 4.10 & 3.39 \\
$p=v_{L}$ & 42.69 & 43.03 & 53.91 & 55.52 \\
$p=v_{L}+1$ & 3.59 & 3.30 & 4.07 & 4.04 \\
$p=v_{L}+2$ & 1.94 & 1.60 & 1.07 & 0.82 \\
$p=1.5 \times v_{L}$ & 1.36 & 0.74 & 0.38 & 0.27 \\
$p=\frac{v_{L}+v_{H}}{2}$ & 0.48 & 0.27 & 0.41 & 0.49 \\
$p=\frac{3}{4} \frac{v_{L}+v_{H}}{2}$ & 0.05 & 0.05 & 0.0 & 0.0 \\
$p=v_{H}$ & 15.40 & 14.52 & 9.29 & 8.31 \\
$\left.p=v_{H}, v_{H}\right)$ and not yet classified & 21.33 & 23.94 & 17.16 & 16.99 \\
$p=v_{H}+2$ & 1.94 & 2.13 & 1.39 & 1.64 \\
$p=v_{L}+v_{H}$ & 1.22 & 1.28 & 0.71 & 0.93 \\
$p=1.5 \times v_{H}$ & 0.48 & 0.53 & 1.61 & 1.97 \\
and not yet classified & 0.0 & 0.0 & 0.05 & 0.11 \\
Total & 8.40 & 7.77 & 5.85 & 5.52 \\
\hline \hline$>v_{H}$ ald & 100.0 & 100.0 & 100.0 & 100.0 \\
\hline \hline
\end{tabular}

Table 16: Distribution of Prices by Treatment (in \%)

Notes: 188 and 183 participants in PROB and DET, respectively. All rounds include data for $3,760(188$ subjects $\times 20$ rounds) and 3,660 (183 subjects $\times 10$ rounds) in PROB and DET, respectively. Last 10 rounds include data for 1,880 $(188$ subjects $\times 10$ rounds) and 1,830 (183 subjects $\times 10$ rounds $)$ in PROB and DET, respectively.

Table 17 shows the distribution of submitted prices for PROB and DET divided into five categories: $p<v_{L}, p=v_{L}, p \in\left(v_{L}, v_{H}\right), p=v_{H}$ and $p>v_{H}$ for each treatment and a series of subsamples. 
The first two rows include all 20 rounds of part 1, the second set of two rows constrains the sample to the last 10 rounds. The third and fourth set consider subjects who never took a risky lottery in part 5 and subjects who took at least one risky lottery in part 5 , respectively.

\begin{tabular}{ccccccc|c}
\hline \hline & & $p<v_{L}$ & $p=v_{L}$ & $p \in\left(v_{L}, v_{H}\right)$ & $p=v_{H}$ & $p>v_{H}$ & Participants \\
\hline \multirow{2}{*}{ All rounds } & PROB & 1.1 & 42.7 & 22.8 & 21.3 & 12.1 & 188 \\
& DET & 4.1 & 53.9 & 15.2 & 17.2 & 9.6 & 183 \\
\hline \multirow{2}{*}{ Last 10 rounds } & PROB & 0.8 & 43.0 & 20.5 & 23.9 & 11.7 & 188 \\
& DET & 3.4 & 55.5 & 13.9 & 17.0 & 10.1 & 183 \\
\hline No risky & PROB & 1.3 & 50.0 & 18.8 & 19.3 & 10.7 & 129 \\
Lottery & DET & 4.8 & 58.8 & 12.8 & 16.1 & 7.5 & 128 \\
\hline At least one & PROB & 0.8 & 26.7 & 31.7 & 25.8 & 15.1 & 59 \\
Risky Lottery & DET & 2.5 & 42.5 & 20.9 & 19.6 & 14.6 & 55 \\
\hline \hline
\end{tabular}

Table 17: Main Treatments: Distribution of Prices in Part 1 (in \%)

Notes: 'All rounds' uses prices submitted for all 20 problems and 'Last 10 rounds' for the last 10 problems subjects faced in part 1. 'No risky lottery' includes subjects who never selected a risky lottery in part 5. 'At least one risky lottery' involves subjects who selected at least one risky lottery in part 5.

Table 17 shows that, when considering all 20 rounds, there are substantially more prices $p=v_{L}$ in DET (53.9 percent) than in PROB (42.7 percent). The numbers are virtually unchanged when we only consider the last 10 rounds. Column (1) of Table 18 presents a random-effects estimation that confirms this treatment effect to be significant. Focusing on just the payoff-maximizing price $p=v_{L}$ we find evidence consistent with the Power of Certainty hypothesis.

Offers of $p=v_{H}$ are 4.1 percentage points higher in PROB than in DET, a difference that increases to 6.9 percentage points in the last 10 rounds. The treatment effect, however, is not statistically significant per Column (2) of Table 18. Consistent with the classification into types, while it may be tempting to attribute $p=v_{H}$ prices in PROB to subjects being risk seeking, the prevalence of such prices in DET, where they are dominated, casts doubt on that interpretation.

Prices that are strictly dominated in both treatments, that is $p \notin\left\{v_{L}, v_{H}\right\}$, are more prevalent in PROB than in DET, with 36.0 (33.0) and 28.9 (27.4) percent such prices in all (the last 10) rounds, respectively. Column (3) of Table 18 shows that the treatment effect is statistically significant. ${ }^{51}$

The differences in submitted prices across treatments can be easily summarized by Figure 6 . We show the cumulative distribution of normalized prices. We compute a normalized price $p^{N}=\frac{p-v_{L}}{v_{H}-v_{L}}$, such

\footnotetext{
${ }^{51}$ Note that there is a class of prices $p \notin\left\{v_{L}, v_{H}\right\}$ that guarantees no losses and could be interpreted as subjects opting out of participating in the problems, namely $p<v_{L}$. There are few such prices in either treatment, though the fraction is a little higher in DET. Nonetheless, $1.1(0.8)$ percent of prices in PROB are such that $p<v_{L}$ in all (the last 10) rounds, while the numbers are 4.1 (3.8) percent in DET. The proportion of subjects who perhaps mistakenly believe that they have to submit a price of $v_{L}+1$ to buy a company of value $v_{L}$ is also small and comparable across treatments, 3.6 (3.3) percent in PROB and 4.1 (4.0) percent in DET in all (the last 10) rounds. The instructions do include a question that checks the understanding (see Instructions Appendix) that submitting $p=v$ guarantees buying a company of value $v$.
} 


\begin{tabular}{|c|c|c|c|}
\hline & $\begin{array}{c}(1) \\
p=v_{L}\end{array}$ & $\begin{array}{c}(2) \\
p=v_{H}\end{array}$ & $p \notin\left\{v_{H}^{(3)}, v_{L}\right\}$ \\
\hline Det & $\begin{array}{l}0.151^{* * *} \\
(0.054)\end{array}$ & $\begin{array}{c}0.002 \\
(0.043)\end{array}$ & $\begin{array}{c}-0.153^{* * *} \\
(0.055)\end{array}$ \\
\hline Num Risky & $\begin{array}{c}-0.095^{* * *} \\
(0.028)\end{array}$ & $\begin{array}{c}0.034 \\
(0.022)\end{array}$ & $\begin{array}{l}0.061^{* *} \\
(0.029)\end{array}$ \\
\hline Num Risky $\times$ Det & $\begin{array}{c}0.035 \\
(0.041)\end{array}$ & $\begin{array}{c}0.008 \\
(0.033)\end{array}$ & $\begin{array}{c}-0.043 \\
(0.042)\end{array}$ \\
\hline Num Errors & $\begin{array}{c}-0.091^{* *} \\
(0.042)\end{array}$ & $\begin{array}{c}-0.003 \\
(0.033)\end{array}$ & $\begin{array}{l}0.094^{* *} \\
(0.042)\end{array}$ \\
\hline Num Errors $\times$ Det & $\begin{array}{c}-0.149^{* * *} \\
(0.058)\end{array}$ & $\begin{array}{c}-0.008 \\
(0.046)\end{array}$ & $\begin{array}{l}0.158^{* * *} \\
(0.059)\end{array}$ \\
\hline Last 10 Periods & $\begin{array}{c}0.007 \\
(0.009)\end{array}$ & $\begin{array}{l}0.052^{\text {*** }} \\
(0.008)\end{array}$ & $\begin{array}{c}-0.059^{* * *} \\
(0.007)\end{array}$ \\
\hline Last 10 Periods $\times$ Det & $\begin{array}{l}0.025^{* *} \\
(0.012)\end{array}$ & $\begin{array}{c}-0.055^{* * *} \\
(0.012)\end{array}$ & $\begin{array}{l}0.030^{* * *} \\
(0.010)\end{array}$ \\
\hline Female & $\begin{array}{c}-0.149^{* * *} \\
(0.041)\end{array}$ & $\begin{array}{c}0.052 \\
(0.032)\end{array}$ & $\begin{array}{c}0.097^{* *} \\
(0.041)\end{array}$ \\
\hline White & $\begin{array}{l}0.118^{* *} \\
(0.049)\end{array}$ & $\begin{array}{c}-0.038 \\
(0.039)\end{array}$ & $\begin{array}{c}-0.080 \\
(0.050)\end{array}$ \\
\hline Young & $\begin{array}{c}-0.026 \\
(0.041)\end{array}$ & $\begin{array}{c}0.009 \\
(0.032)\end{array}$ & $\begin{array}{c}0.017 \\
(0.041)\end{array}$ \\
\hline Low Schooling & $\begin{array}{c}-0.067 \\
(0.042)\end{array}$ & $\begin{array}{c}0.061^{*} \\
(0.033)\end{array}$ & $\begin{array}{c}0.006 \\
(0.043)\end{array}$ \\
\hline Constant & $\begin{array}{l}0.548^{* * *} \\
(0.063)\end{array}$ & $\begin{array}{l}0.140^{* * *} \\
(0.050)\end{array}$ & $\begin{array}{l}0.311^{* * *} \\
(0.064)\end{array}$ \\
\hline Observations & 7420 & 7420 & 7420 \\
\hline
\end{tabular}

Table 18: Main Treatments: Random Effects Estimations Output using Part-1 Prices

Notes: The dependent variable takes value 1 if: (1) $p=v_{L}$, (2) $p=v_{H}$, (3) $p \notin\left\{v_{L}, v_{H}\right\}$. Det is a dummy variable that takes value 1 if the observation corresponds to the deterministic treatment. Num Risky and Num Errors are individual-specific. Num Risky is the number of risky lotteries the subject chooses in part 5 (from 0 to 3 ). Num Errors is the number of errors the subject made in the part 1 instructions (from 0 to 2). Last 10 Periods is a dummy variable that takes value 1 if the observation corresponds to the last 10 periods of part 1. Female, White, Young and Low Schooling are a dummies that take value 1, respectively, if the subject's gender is female, the reported ethnicity is white, their age is below the median age of 32, and their schooling if their education level is 'Some College' or lower. There are 371 individuals (188 in PROB and 183 in DET) and 20 observations in part 1 per individual for a total of 7,420 observations. 


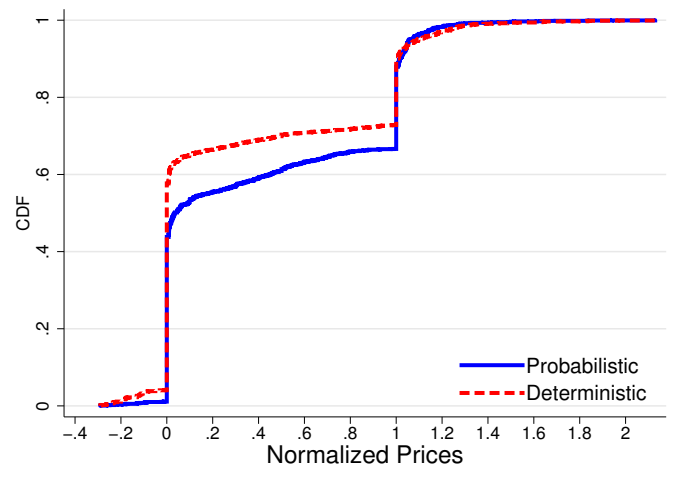

(a) All rounds

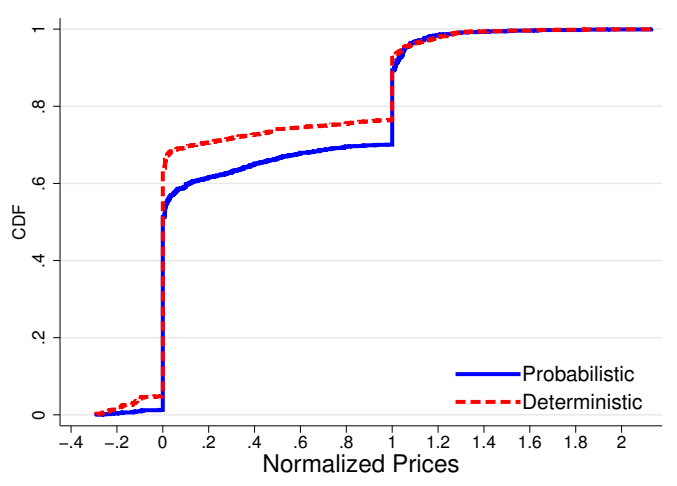

(c) Subjects who never took a risk

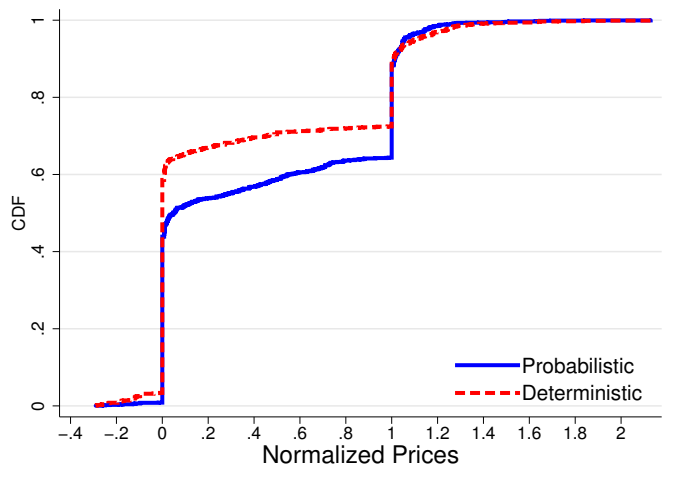

(b) Last 10 rounds

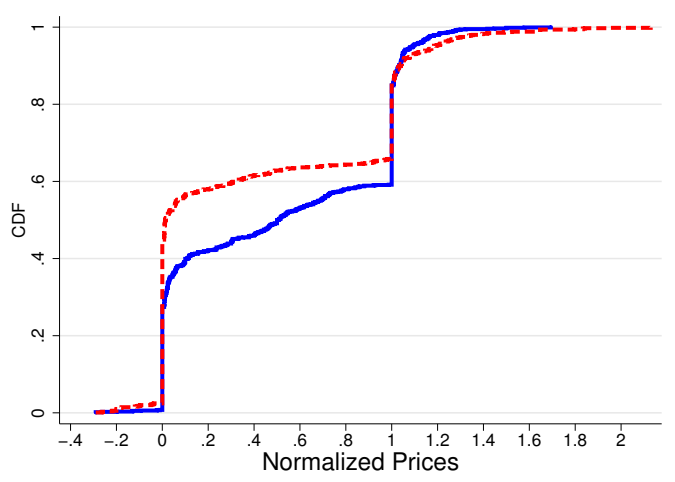

(d) Subjects who took risk once or more

Figure 6: Main Treatments Part 1-Distribution of Normalized Prices by Treatment

Notes: Normalized Prices $\left(p^{N}\right)$ are computed for each question and subject in the following manner: $p^{N}=\frac{p-v_{L}}{v_{H}-v_{L}}$, where $p$ is the price submitted by the subject for a question with values $\left\{v_{L}, v_{H}\right\}$. Notice that if $p=v_{H}$, then $p^{N}=1$ and if $p=v_{L}$, then $p^{N}=0$. Values of $p^{N} \in(0,1)$ indicate subjects submitting dominated prices in the interval $p \in\left(v_{L}, v_{H}\right)$. The case $p^{N}<0$, corresponds with $p<v_{L}$ and, finally, the case $p^{N}>1$ takes place if $p>v_{H}$. 'All rounds' uses the answers submitted for all 20 problems and 'Last 10 rounds' for the last 10 problems subjects faced in part 1. 'Subjects who never took a risk' includes subjects who never selected a risky lottery in part 5. 'Subjects who took risk once or more' involves subjects who selected at least one risky lottery in part 5. 


\begin{tabular}{|c|c|c|c|c|c|c|}
\hline & $\begin{array}{c}(1) \\
p=v_{L}\end{array}$ & $\begin{array}{c}(2) \\
p=v_{L}\end{array}$ & $\begin{array}{c}(3) \\
p=v_{H}\end{array}$ & $\begin{array}{c}(4) \\
p=v_{H}\end{array}$ & $p \notin\left\{v_{H}, v_{L}\right\}$ & $p \notin\left\{v_{H}^{(6)}, v_{L}\right\}$ \\
\hline Det & $\begin{array}{c}-0.057 \\
(0.036)\end{array}$ & $\begin{array}{c}0.045 \\
(0.046)\end{array}$ & $\begin{array}{l}0.199^{* * *} \\
(0.059)\end{array}$ & $\begin{array}{c}0.059 \\
(0.074)\end{array}$ & $\begin{array}{c}-0.142^{* *} \\
(0.055)\end{array}$ & $\begin{array}{c}-0.105 \\
(0.066)\end{array}$ \\
\hline Num Risky & $\begin{array}{c}-0.002 \\
(0.019)\end{array}$ & $\begin{array}{c}0.021 \\
(0.019)\end{array}$ & $\begin{array}{c}-0.069^{* *} \\
(0.031)\end{array}$ & $\begin{array}{r}-0.054^{*} \\
(0.030)\end{array}$ & $\begin{array}{l}0.072^{* *} \\
(0.029)\end{array}$ & $\begin{array}{c}0.033 \\
(0.027)\end{array}$ \\
\hline Num Risky × Det & $\begin{array}{c}-0.014 \\
(0.028)\end{array}$ & $\begin{array}{c}-0.036 \\
(0.027)\end{array}$ & $\begin{array}{c}0.048 \\
(0.045)\end{array}$ & $\begin{array}{c}0.041 \\
(0.044)\end{array}$ & $\begin{array}{c}-0.034 \\
(0.042)\end{array}$ & $\begin{array}{c}-0.004 \\
(0.039)\end{array}$ \\
\hline Num Errors & $\begin{array}{l}0.056^{* *} \\
(0.028)\end{array}$ & $\begin{array}{l}0.079^{* * *} \\
(0.027)\end{array}$ & $\begin{array}{l}-0.150^{* * *} \\
(0.046)\end{array}$ & $\begin{array}{l}-0.137^{* * *} \\
(0.044)\end{array}$ & $\begin{array}{l}0.094^{* *} \\
(0.043)\end{array}$ & $\begin{array}{c}0.059 \\
(0.040)\end{array}$ \\
\hline Num Errors $\times$ Det & $\begin{array}{r}-0.070^{*} \\
(0.039)\end{array}$ & $\begin{array}{l}-0.095^{* *} \\
(0.039)\end{array}$ & $\begin{array}{c}-0.092 \\
(0.063)\end{array}$ & $\begin{array}{c}-0.033 \\
(0.063)\end{array}$ & $\begin{array}{l}0.162^{* * *} \\
(0.060)\end{array}$ & $\begin{array}{l}0.128^{* *} \\
(0.056)\end{array}$ \\
\hline Female & $\begin{array}{c}-0.014 \\
(0.027)\end{array}$ & $\begin{array}{c}-0.001 \\
(0.027)\end{array}$ & $\begin{array}{r}-0.080^{*} \\
(0.044)\end{array}$ & $\begin{array}{c}-0.042 \\
(0.043)\end{array}$ & $\begin{array}{l}0.094^{* *} \\
(0.042)\end{array}$ & $\begin{array}{c}0.043 \\
(0.039)\end{array}$ \\
\hline White & $\begin{array}{c}-0.006 \\
(0.033)\end{array}$ & $\begin{array}{c}-0.024 \\
(0.032)\end{array}$ & $\begin{array}{c}0.101^{*} \\
(0.054)\end{array}$ & $\begin{array}{l}0.080 \\
(0.052)\end{array}$ & $\begin{array}{r}-0.095^{*} \\
(0.051)\end{array}$ & $\begin{array}{r}-0.056 \\
(0.047)\end{array}$ \\
\hline Young & $\begin{array}{c}0.010 \\
(0.027)\end{array}$ & $\begin{array}{c}0.004 \\
(0.027)\end{array}$ & $\begin{array}{c}-0.023 \\
(0.044)\end{array}$ & $\begin{array}{r}-0.008 \\
(0.043)\end{array}$ & $\begin{array}{c}0.013 \\
(0.042)\end{array}$ & $\begin{array}{c}0.004 \\
(0.038)\end{array}$ \\
\hline Low Schooling & $\begin{array}{r}-0.035 \\
(0.028)\end{array}$ & $\begin{array}{c}-0.030 \\
(0.027)\end{array}$ & $\begin{array}{c}-0.001 \\
(0.046)\end{array}$ & $\begin{array}{c}-0.001 \\
(0.044)\end{array}$ & $\begin{array}{c}0.036 \\
(0.043)\end{array}$ & $\begin{array}{c}0.031 \\
(0.039)\end{array}$ \\
\hline$V_{L}($ Part 1$)$ & & $\begin{array}{l}0.223^{* * *} \\
(0.041)\end{array}$ & & $\begin{array}{l}0.155^{* *} \\
(0.066)\end{array}$ & & $\begin{array}{l}-0.378^{* * *} \\
(0.059)\end{array}$ \\
\hline$V_{L}($ Part 1$) \times \operatorname{Det}$ & & $\begin{array}{l}-0.230^{* * *} \\
(0.057)\end{array}$ & & $\begin{array}{l}0.187^{* *} \\
(0.091)\end{array}$ & & $\begin{array}{c}0.043 \\
(0.082)\end{array}$ \\
\hline Constant & $\begin{array}{l}0.144^{* * *} \\
(0.042)\end{array}$ & $\begin{array}{c}0.057 \\
(0.044)\end{array}$ & $\begin{array}{l}0.637^{* * *} \\
(0.068)\end{array}$ & $\begin{array}{l}0.561^{* * *} \\
(0.070)\end{array}$ & $\begin{array}{l}0.219^{* * *} \\
(0.064)\end{array}$ & $\begin{array}{l}0.382^{* * *} \\
(0.063)\end{array}$ \\
\hline Observations & 1855 & 1855 & 1855 & 1855 & 1855 & 1855 \\
\hline
\end{tabular}

Table 19: Main Treatments: Random Effects Estimations Output using Part-2 Prices

Notes: The dependent variable takes value 1 if the subject selects in (1) and (2) $p=v_{L}$, in (3) and (4) $p=v_{H}$ and in (5) and (6) $p \notin\left\{v_{L}, v_{H}\right\}$. The regression includes all 5 periods of part 2. Columns (2), (4) and (6) control for whether the subject was classified as submitting $p=v_{L}$ in the last five rounds of part $1\left(V_{L}\right)$ and its interaction with Det. Det is a dummy variable that takes value 1 if the observation corresponds to the deterministic treatment. Num Risky and Num Errors are individual-specific. Num Risky is the number of risky lotteries the subject chose in part 5 (from 0 to 3). Num Errors is the number of errors the subject made in the part 1 instructions (from 0 to 2). Female, White, Young and Low Schooling are a dummies that take value 1 , respectively, if the subject's gender is female, the reported ethnicity is white, their age is below the median age of 32 , and their schooling if their education level is 'Some College' or lower. 


\begin{tabular}{cccccc|c}
\hline \hline Part 1 Types & $V_{H}$ & $V_{L}$ & Mix & Dom & Residual & Participants \\
\hline PROB & 12.2 & 31.9 & 20.7 & 27.7 & 7.5 & 188 \\
DET & 9.8 & 47.5 & 9.8 & 19.3 & 13.7 & 183 \\
\hline \hline
\end{tabular}

Table 20: Part 1 Type Classification [as \% of participants]

Notes: Subjects are classified as belonging to a type if their submitted price corresponds to the same type in the last 5 rounds of part 1 (rounds 16-20). $V_{L}: p=v_{L}$. $V_{H}: p=v_{H}$. Mix: $p=v_{L}$ or $p=v_{H}$ (and at least one of each). Dom: $p \notin\left\{v_{L}, v_{H}\right\}$. Residual: all other subjects.

that $p^{N}=0$ indicates $p=v_{L}$, and $p^{N}=1$ corresponds to $p=v_{H}$. The figure shows the cumulative distribution of normalized prices in part 1 for all rounds (panel a) and the last 10 rounds (panel b). The distribution of normalized prices in PROB first order stochastically dominates the distribution in the DET and the difference is statistically significant. ${ }^{52}$ Panels $\mathrm{c}$ and d confirm that the results hold if we distinguish between subjects who did and did not select at least one risky lottery in part 5. (The estimates reported in Table 18 also control for part 5 choices).

Finally, Table 19 reports findings in part 2. The left-hand side variables take value 1 if $p=v_{L}$ (the first two columns) or if $p=v_{H}$ (last two columns) and the unit of observation is each question of part 2. Columns (1), (3) and (5) present results that use the same controls as in Table 18. There is no treatment effect in $p=v_{L}(1)$, but there is a significantly higher likelihood of observing $p=v_{H}$ in DET per (3). When we combine findings of part 1 with those of part 2 (see columns (2), (4) and (6)), we find that being classified as $V_{L}$ in part 1 increases the chances of submitting $p=v_{L}$ in part 2 of PROB, but not in DET (where the addition of 0.223 and -0.230 is essentially zero). We also find that being classified as $V_{L}$ in part 1 significantly increases the chances of submitting $p=v_{H}$ in both treatments (by a positive coefficient 0.155 ), but there is an additional effect in DET that is significant (a coefficient of 0.187).

In summary, the evidence on prices rather than classification of subjects confirms the results from Section 3, and mirrors that risk seeking preferences cannot account for the findings. That is, using prices instead of types in part 1 confirms the evidence for the Power of Certainty.

We can also use prices, rather than types, to assess the relative role of $P o C$. For that, we first provide evidence on prices in the first 25 rounds of the ONEFIRM treatment.

When considering all 25 rounds of the ONEFIRM treatment, 73.9 percent of prices are $p=v, 8.1$ percent are $p<v$ and 18 percent are $p>v .^{53}$

\footnotetext{
${ }^{52}$ We test for first-order stochastic dominance using the test in Barrett and Donald (2003). The test consists of two steps. We first test the null hypothesis that the distribution in the deterministic treatment either first order stochastically dominates or is equal to the distribution in the probabilistic treatment. We reject this null hypothesis using all rounds or the last 10 rounds, the corresponding $p$-value is 0 in both cases. We then test the null hypothesis that the distribution in the probabilistic treatment first order stochastically dominates the distribution in the deterministic treatment. We cannot reject the null in this case, with a corresponding $p$-value of 0.817 using all rounds and 0.239 using the last 10 rounds.

${ }^{53}$ The numbers for the last 10 of the 25 rounds are very similar, $74.3,7.1$ and 18.6 percent, respectively. When
} 


\begin{tabular}{ccccccccc}
\hline \hline & & $V_{L}^{80} V_{H}^{80} / V^{80}$ & $\left\{\right.$ Mix $\left.^{80} V_{H}^{80}, V_{H}^{80} V_{H}^{80}\right\}$ & Focal $^{80}$ & Dom $^{80}$ & Residual $^{80}$ & Participants $^{2}$ \\
\hline \multirow{2}{*}{ All } & PROB & 26.6 & 22.9 & 15.4 & 27.1 & 8.0 & 188 \\
& DET & 49.2 & 15.3 & 4.9 & 21.3 & 9.3 & 183 \\
& ONEFIRM & 67.1 & - & - & 20.5 & 12.4 & 428 \\
\hline \hline No & PROB & 32.6 & 21.7 & 16.3 & 20.1 & 9.3 & 129 \\
Risky & DET & 52.4 & 14.8 & 6.3 & 15.6 & 10.9 & 128 \\
Lottery & ONEFIRM & 71.8 & - & - & 18.1 & 10.1 & 276 \\
\hline \hline At least one & PROB & 13.5 & 25.4 & 13.6 & 42.4 & 5.1 & 59 \\
Risky & DET & 41.8 & 16.4 & 1.8 & 34.5 & 5.5 & 55 \\
Lottery & ONEFIRM & 58.6 & - & - & 25.0 & 16.4 & 152 \\
\hline \hline
\end{tabular}

Table 21: Part 1 and Part 2 Type Classification Allowing for Deviations [as \% of participants]

Notes: Types are defined based on the prices $p_{1}$ submitted in at least 80 percent of the last five rounds of part 1 and $p_{2}$ submitted in at least 80 percent of the five rounds of part 2 . Type $V_{L}^{80} V_{H}^{80}: p_{1}=v_{L}$ and $p_{2}=v_{H}$. Type $\left\{M i x^{80} V_{H}^{80}, V_{H}^{80} V_{H}^{80}\right\}$ : $p_{1} \in\left\{v_{L}, v_{H}\right\}$ and at least one $p_{1}=v_{H}$ and $p_{2}=v_{H}$ and not classified as $V_{L}^{80} V_{H}^{80}$. Type 'Focal ${ }^{80}$ ': $p_{1}, p_{2} \in\left\{v_{L}, v_{H}\right\}$ and at least one $p_{2}=v_{L}$ (corresponds to $V_{L}^{80} V_{L}^{80}, V_{L}^{80} \mathrm{Mix}^{80}, \mathrm{Mix}^{80} V_{L}^{80}, \mathrm{Mix}^{80}{ }_{\mathrm{Mix}}^{80}, V_{H}^{80} V_{L}^{80}$, or $V_{H}^{80} \mathrm{Mix}^{80}$. Type 'Dom ${ }^{80}$ ': $p_{1}, p_{2} \notin\left\{v_{L}, v_{H}\right\}$. Residual ${ }^{80}$ : All remaining subjects. In onEfiRm subjects are classified as $V^{80}$ if they submit $p=v$ in at least 80 percent of rounds $16-25$, and as 'Dom ${ }^{80}$ ' if $p \neq v$ in at least 80 percent of rounds $16-25$, with Residual ${ }^{80}$ being the remaining subjects. In all classifications, since there is a potential for a subject to be classified as two different types (in two different columns), we break ties such that we classify the subject in the left column.

\section{B Classification using Part 1 and Robustness of Classifications}

First, we show the classification of subjects into types using only part 1, see Table 20.

The first robustness of classification we consider weakens the requirement that subjects submit the type specific price in each of the last 5 rounds of part 1 and all five rounds of part 2. Subjects only have to submit the type specific price in 4 of the 5 rounds (that is 80 percent of the prices have to be type specific). For each subject we first assess whether they can be classified as $V_{L}^{80} V_{H}^{80}$. We then check for the remainder, whether they can be classified as $M i x^{80} V_{H}^{80}$ or $V_{H}^{80} V_{H}^{80}$. And subsequently for the remainder we assess whether they can be classified as Focal ${ }^{80}$, and then as Dominated ${ }^{80}$. All residual subjects are classified as Residual ${ }^{80}$, see Table 21. Figure 7 shows the evolution of types using this more lenient classification.

Table 22 shows that the main result of Table 3 is robust to using this more lenient classification. Specifically, subjects in DET are significantly more likely to be classified as $V_{L}^{80} V_{H}^{80}$ compared to subjects in PROB. While subjects who chose risky lotteries in part 5 are less likely to be so classified,

we distinguish between subjects in the probabilistic and the deterministic version of ONEFIRM, the numbers are very similar. This is not surprising given that in the first 25 rounds the only difference between the two treatments is that subjects have seen instructions for either treatment, but have not actually played in those treatments. In the probabilistic version 74.9 (74.9) percent of prices are $p=v, 9.7(8.5)$ are $p<v$ and $15.4(16.6)$ are $p>v$ when we consider all 25 (the last 10) rounds. In the deterministic version, the numbers are 72.9 (73.6) percent of prices are $p=v, 6.5(5.7)$ are $p<v$ and 20.6 (20.7) are $p>v$ when we consider all 25 (the last 10) rounds. Furthermore, a random-effects panel regression with a dummy that takes value 1 if $p=v$ on the left-hand side and a treatment dummy on the right-hand side shows that the treatment dummy is not significant if we look at all rounds ( $\mathrm{p}$-value 0.576 ) or at the last 10 rounds (p-value 0.723 ). 


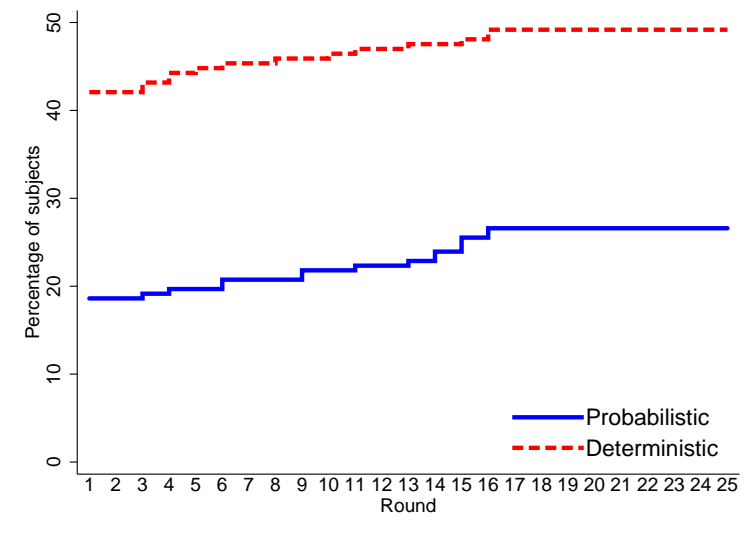

(a) $p=v_{L}: n$ to $15 \& V_{L}^{80} V_{H}^{80}$.

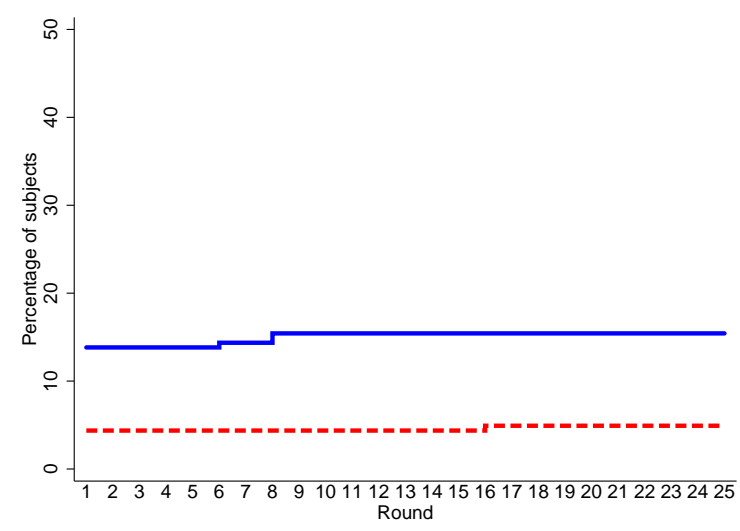

(c) $p \in\left\{v_{L}, v_{H}\right\}: n$ to $15 \&$ Focal $^{80}$.

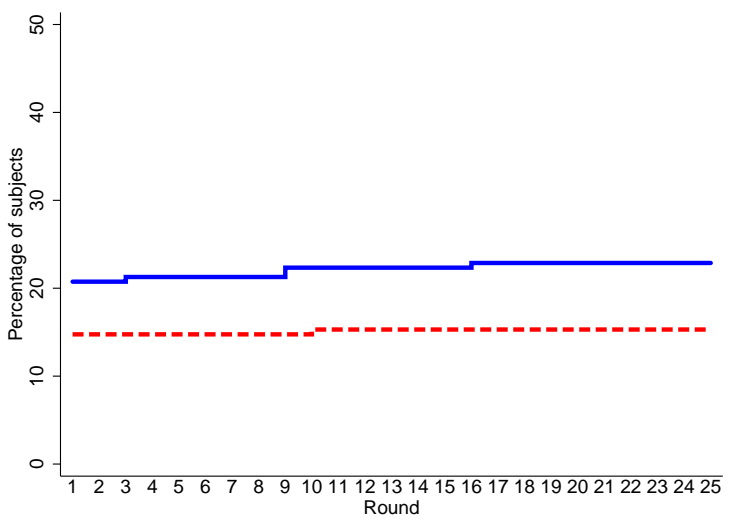

(b) $p \in\left\{v_{L}, v_{H}\right\}: n$ to $15 \&\left\{\operatorname{Mix}^{80} V_{H}^{80}, V_{H}^{80} V_{H}^{80}\right\}$.

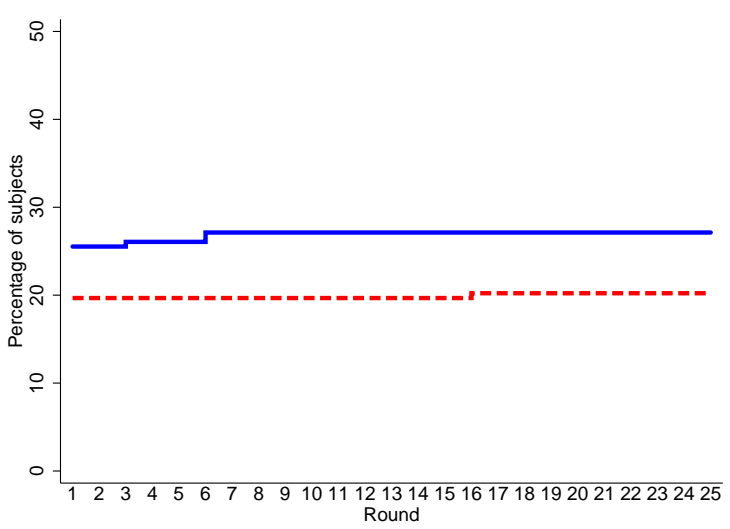

(d) $p \notin\left\{v_{L}, v_{H}\right\}: n$ to $15 \&$ Dominated $^{80}$.

Figure 7: Evolution of Types (Part 1 \& Part 2) Allowing for Deviations.

Notes: Percent of subjects who submit the described part- 1 price $p_{1}$ in 80 percent of the rounds from round $n$ to 20 and part- 2 price $p_{2}$ in 80 percent of the rounds in part 2 by treatment. 


\begin{tabular}{lccccc}
\hline & $(1)$ & $(2)$ & $(3)$ & $(4)$ & $(5)$ \\
& $V^{80} V_{H}^{80}$ & $\left\{M i x^{80} V_{H}^{80}, V_{H}^{80} V_{H}^{80}\right\}$ & Focal $^{80}$ & Dom $^{80}$ & Dom $^{80}$ or Res $^{80}$ \\
\hline Det & $0.272^{* * *}$ & -0.085 & $-0.072^{*}$ & $-0.125^{* *}$ & $-0.115^{*}$ \\
Num Risky & $(0.063)$ & $(0.055)$ & $(0.042)$ & $(0.057)$ & $(0.062)$ \\
& $-0.062^{*}$ & 0.011 & -0.013 & $0.080^{* * *}$ & $0.064^{* *}$ \\
Num Risky $\times$ Det & $(0.033)$ & $(0.029)$ & $(0.022)$ & $(0.030)$ & $(0.032)$ \\
& 0.030 & 0.023 & 0.004 & -0.029 & -0.057 \\
Num Errors & $(0.048)$ & $(0.042)$ & $(0.032)$ & $(0.044)$ & $(0.047)$ \\
Num Errors $\times$ Det & $-0.124^{* *}$ & -0.048 & $0.069^{* *}$ & 0.059 & $0.103^{* *}$ \\
Female & $(0.048)$ & $(0.043)$ & $(0.032)$ & $(0.044)$ & $(0.048)$ \\
White & $-0.112^{*}$ & 0.021 & $-0.090^{* *}$ & $0.157^{* *}$ & $0.181^{* * *}$ \\
& $(0.067)$ & $(0.060)$ & $(0.045)$ & $(0.061)$ & $(0.066)$ \\
Young & $-0.103^{* *}$ & 0.033 & -0.032 & $0.073^{*}$ & $0.103^{* *}$ \\
& $(0.047)$ & $(0.042)$ & $(0.032)$ & $(0.043)$ & $(0.046)$ \\
Low Schooling & $0.132^{* *}$ & 0.001 & -0.023 & $-0.106^{* *}$ & $-0.110^{*}$ \\
Constant & $(0.057)$ & $(0.051)$ & $(0.038)$ & $(0.052)$ & $(0.056)$ \\
& -0.031 & -0.001 & 0.009 & 0.046 & 0.023 \\
Observations & $(0.047)$ & $(0.042)$ & $(0.032)$ & $(0.043)$ & $(0.046)$ \\
\hline & -0.033 & 0.038 & -0.044 & 0.015 & 0.039 \\
& $(0.049)$ & $(0.043)$ & $(0.033)$ & $(0.044)$ & $(0.048)$ \\
& $0.339^{* * *}$ & $0.209^{* * *}$ & $0.181^{* * *}$ & $0.212^{* * *}$ & $0.270^{* * *}$ \\
& $(0.072)$ & $(0.064)$ & $(0.049)$ & $(0.066)$ & $(0.071)$ \\
\hline
\end{tabular}

Table 22: Main Treatments: Estimation output using last 5 rounds of part 1 and part 2 for the classification of types that allows for one deviation

Notes: Results from a linear regression. The dependent variable takes value 1 if the subject is classified as (1) $V_{L}^{80} V_{H}^{80},(2)$ $\left\{M i x^{80} V_{H}^{80}, V_{H}^{80} V_{H}^{80}\right\}$, (3) Focal ${ }^{80}$, (4) $\operatorname{Dom}^{80}$, (5) $\mathrm{Dom}^{80}$ or Res ${ }^{80}$. A subject is classified as $V_{L}^{80} V_{H}^{80}$ if they submitted $p=v_{L}$ in at least 80 percent of the last 5 periods of part 1 and $p=v_{H}$ in at least 80 percent of the periods of part 2 . A subject is classified as $M i x^{80} V_{H}^{80}$ if they mix between $p=v_{L}$ and $p=v_{H}$ in the last 5 periods of part 1, selecting at least one of the two and not being previously classified as $V_{L}^{80}$, and if they select $p=v_{H}$ in at least 80 percent of the periods of part 2 . The other definitions of types are adjusted accordingly. Det is a dummy variable that takes value 1 if the observation corresponds to the deterministic treatment. Num Risky and Num Errors are individual-specific. Num Risky is the number of risky lotteries the subject chooses in part 5 (from 0 to 3). Num Errors is the number of errors the subject made in the part 1 instructions (from 0 to 2). Female, White, Young and Low Schooling are a dummies that take value 1, respectively, if the subject's gender is female, the reported ethnicity is white, their age is below the median age of 32, and their schooling if their education level is 'Some College' or lower.

there is no significant treatment effect even though risk preferences have no bearing in DET. In addition, subjects who took risks in part 5 are more likely to be classified as the Dominated ${ }^{80}$ type or as either Dominated ${ }^{80}$ or Residual ${ }^{80}$ type, further casting doubt that risk-seeking preferences are responsible for a correlation between a $V_{L}^{80} V_{H}^{80}$ classification and part 5 choices.

Table 21 also shows the classification of subjects in the first two parts of the ONEFIRM treatments. In ONEFIRM, a subject is classified as $V^{80}$ if they submit $p=v$ in at least 80 percent of the rounds 16-25, among the remainder, subjects are classified as $D o m^{80}$, if $p \neq v$ in at least 80 percent of rounds 16-25, and Residual ${ }^{80}$ contains the remaining subjects.

The Power of Certainty is not only robust to the more lenient classification of types, but so is its quantitative importance. We can think of the difference in between 67.1 and 26.6 percent as what used to be attributed to computational complexity. However, 55.8 percent of that total effect can 


\begin{tabular}{ccccccccc}
\hline \hline & & $V_{L}^{A} V_{H}^{A} / V^{A}$ & $\left\{M i x^{A} V_{H}^{A}, V_{H}^{A} V_{H}^{A}\right\}$ & Focal $^{A}$ & Dom $^{A}$ & Residual $^{A}$ & Participants $^{2}$ \\
\hline \multirow{2}{*}{ All } & PROB & 21.8 & 26.6 & 19.1 & 17.6 & 14.9 & 188 \\
& DET & 45.9 & 18.0 & 8.2 & 11.5 & 16.4 & 183 \\
& ONEFIRM & 66.8 & - & - & 12.9 & 20.3 & 428 \\
\hline \hline No & PROB & 27.1 & 27.1 & 18.6 & 12.4 & 14.7 & 129 \\
Risky & DET & 47.7 & 17.2 & 9.4 & 8.6 & 17.2 & 128 \\
Lottery & ONEFIRM & 72.5 & - & - & 12.3 & 15.2 & 276 \\
\hline \hline At least one & PROB & 10.2 & 25.4 & 20.3 & 28.8 & 15.3 & 59 \\
Risky & DET & 41.8 & 20.0 & 5.5 & 18.2 & 14.5 & 55 \\
Lottery & ONEFIRM & 56.6 & - & - & 13.8 & 29.6 & 152 \\
\hline \hline
\end{tabular}

Table 23: Part 1 and Part 2 Type Classification Allowing for Small Overbidding [as \% of participants]

Notes: Adjusted Types are defined based on the prices $p_{1}$ submitted in the last five rounds of part 1 and $p_{2}$ submitted in all five rounds of part 2. Type $V_{L}^{A} V_{H}^{A}: p_{1} \in P_{L}=\left[v_{L}, v_{L}+2\right]$ and $p_{2} \in P_{H}=\left[v_{H}, v_{H}+2\right]$. Type $\left\{M i x^{A} V_{H}^{A}, V_{H}^{A} V_{H}^{A}\right\}: p_{1} \in\left\{P_{L}, P_{H}\right\}$ and at least one $p_{1} \in P_{H}$ and $p_{2} \in P_{H}$ and not classified as $V_{L}^{A} V_{H}^{A}$. Type 'Focal ${ }^{A}$ ': $p_{1}, p_{2} \in\left\{P_{L}, P_{H}\right\}$ and at least one $p_{2} \in P_{L}$ (corresponds to $V_{L}^{A} V_{L}^{A}, V_{L}^{A} M i x^{A}, M_{i x}^{A} V_{L}^{A}, M i x^{A} M i x{ }^{A}, V_{H}^{A} V_{L}^{A}$, or $V_{H}^{A} M i x^{A}$. Type 'Dom ${ }^{A}: p_{1}, p_{2} \notin\left\{v_{L}, v_{H}\right\}$ and not classified as another type. Residual ${ }^{A}$ : All remaining subjects. In ONEFIRM subjects are classified as $V^{A}$ if they submit $p \in P=[v, v+2]$ in all rounds 16-25, and as ' $\operatorname{Dom}^{A}$ ' if $p \neq v$ in all of rounds 16-25 (and not classified as $V^{A}$ ), with Residual ${ }^{A}$ being the remaining subjects.

actually be attributed to the lack of the $P o C$, and only the remainder to complexity.

In a second robustness exercise we classify a subject as submitting $p=v_{L}$ and $p=v_{H}$ if they submit $p \in\left[v_{L}, v_{L}+2\right]$ and $p \in\left[v_{H}, v_{H}+2\right]$. While we had a question in the instructions testing the understanding that $p=v$ would purchase a firm of value $v$ (see Instructions Appendix), some subjects may still be confused and pay a price that is slightly too high. Again, if we used this second classification, we would reach similar conclusions. In this case, we can think of the difference between 66.8 and 21.8 percent as what used to be capture complexity, but 53.6 percent of the total difference can be attributed to $P_{o} C$.

\section{Robustness to Binary Lottery Choice Controls}

In this section we show that the main results in Table 3 is robust when instead of linearly controlling for the number of risky lotteries a subject chose, we use a dummy whether the subject chose at least one risky lottery in part 5 . The results are shown in Table 24.

\section{Advice}

In Table 25 we provide the full results as to the numerical price recommendation subjects provided as a function of the outcomes mentioned in the Advice. 


\begin{tabular}{lccccc}
\hline & $(1)$ & $(2)$ & $(3)$ & $(4)$ & $(5)$ \\
& $V_{L} V_{H}$ & $\left\{M i x V_{H}, V_{H} V_{H}\right\}$ & Focal & Dom & Dom or Res \\
\hline Det & $0.229^{* * *}$ & $-0.098^{*}$ & -0.050 & -0.073 & -0.081 \\
Risky & $(0.062)$ & $(0.058)$ & $(0.049)$ & $(0.054)$ & $(0.065)$ \\
& $-0.160^{* *}$ & -0.033 & 0.004 & $0.192^{* * *}$ & $0.189^{* * *}$ \\
Risky $\times$ Det & $(0.068)$ & $(0.064)$ & $(0.054)$ & $(0.059)$ & $(0.071)$ \\
& 0.131 & 0.048 & -0.031 & -0.087 & -0.148 \\
Num Errors & $(0.097)$ & $(0.092)$ & $(0.077)$ & $(0.085)$ & $(0.102)$ \\
& $-0.101^{* *}$ & -0.063 & 0.048 & $0.074^{*}$ & $0.116^{* *}$ \\
Num Errors $\times$ Det & $(0.046)$ & $(0.044)$ & $(0.037)$ & $(0.040)$ & $(0.049)$ \\
Female & -0.094 & 0.025 & $-0.096^{*}$ & 0.076 & $0.164^{* *}$ \\
& $(0.064)$ & $(0.061)$ & $(0.051)$ & $(0.056)$ & $(0.068)$ \\
White & $-0.133^{* * *}$ & 0.044 & -0.005 & $0.070^{*}$ & $0.094^{* *}$ \\
& $(0.045)$ & $(0.043)$ & $(0.036)$ & $(0.039)$ & $(0.047)$ \\
Young & $0.095^{*}$ & 0.019 & -0.011 & $-0.092^{*}$ & $-0.103^{*}$ \\
& $(0.055)$ & $(0.052)$ & $(0.044)$ & $(0.048)$ & $(0.058)$ \\
Low Schooling & -0.024 & 0.004 & -0.006 & 0.025 & 0.026 \\
Constant & $(0.045)$ & $(0.043)$ & $(0.036)$ & $(0.039)$ & $(0.047)$ \\
& 0.009 & 0.031 & -0.053 & 0.027 & 0.013 \\
\hline Observations & $(0.047)$ & $(0.044)$ & $(0.037)$ & $(0.041)$ & $(0.049)$ \\
\hline
\end{tabular}

Table 24: Main Treatments: Estimation output using last 5 rounds of part 1 and part 2 for the classification of types and using a discrete dummy to control for part 5 choices

Notes: Results from a linear regression. The dependent variable takes value 1 if the subject is classified as $(1) V_{L} V_{H},(2)\left\{\right.$ Mix $V_{H}$, $V_{H} V_{H}$ \}, (3) Focal, (4) Dom, (5) Dom or Res. Det is a dummy variable that takes value 1 if the observation corresponds to the deterministic treatment. Num Risky and Num Errors are individual-specific. Risky is a dummy that takes value 1 if the subject took a risky lottery in part 5 and zero otherwise. Num Errors is the number of errors the subject made in the part 1 instructions (from 0 to 2). Female, White, Young and Low Schooling are a dummies that take value 1, respectively, if the subject's gender is female, the reported ethnicity is white, their age is below the median age of 32, and their schooling if their education level is 'Some College' or lower. 


\begin{tabular}{|c|c|c|c|c|c|c|c|c|}
\hline \multirow{2}{*}{\multicolumn{2}{|c|}{ Part 3 Recommendation }} & \multicolumn{6}{|c|}{ Advice: Outcomes Mentioned } & \multirow[b]{2}{*}{ Sum } \\
\hline & & None & All & Large Gain & Large Loss & $p=v_{L}$ & Error & \\
\hline \multirow{6}{*}{ PROB } & $v_{L}$ & 8.5 & 20.2 & 0.5 & 10.7 & 2.7 & 0.5 & 43.1 \\
\hline & $v_{H}$ & 8.5 & 1.6 & 10.7 & 1.6 & 0.0 & 2.1 & 24.5 \\
\hline & $\operatorname{Mix}\left\{v_{L}, v_{H}\right\}$ & 1.1 & 4.3 & 1.0 & 1.1 & 0.0 & 0.5 & 8.0 \\
\hline & Dom & 12.8 & 0.5 & 0.5 & 0.0 & 0.5 & 0.6 & 14.9 \\
\hline & Unclassified & 9.5 & 0.0 & 0.0 & 0.0 & 0.0 & 0.0 & 9.5 \\
\hline & Sum & 40.4 & 26.6 & 12.8 & 13.3 & 3.2 & 3.7 & 100.0 \\
\hline \multirow{6}{*}{ DET } & $v_{L}$ & 5.5 & 51.4 & 0.0 & 3.3 & 3.8 & 0.0 & 63.9 \\
\hline & $v_{H}$ & 9.8 & 0.0 & 4.9 & 0.0 & 0.0 & 2.7 & 17.5 \\
\hline & $\operatorname{Mix}\left\{v_{L}, v_{H}\right\}$ & 0.0 & 0.0 & 0.0 & 0.0 & 0.0 & 0.0 & 0.0 \\
\hline & Dom & 8.7 & 0.6 & 0.5 & 0.0 & 0.0 & 0.5 & 10.4 \\
\hline & Unclassified & 5.4 & 0.5 & 0.6 & 0.6 & 0.5 & 0.6 & 8.2 \\
\hline & Sum & 29.5 & 52.5 & 6.0 & 3.8 & 4.4 & 3.8 & 100.0 \\
\hline
\end{tabular}

Table 25: Part 3 (Recommendation) v. Part 3 (Advice Outcomes Categories)

Notes: 188 participants in РROB and 183 in DET. There are four outcomes $(v, p)$ where $v, p \in\left\{v_{L}, v_{H}\right\}$. All (none) are subjects who mentioned all (none of the) four outcomes in any form. Large Gain: Subjects who mention either $\left\{\left(v_{L}, v_{L}\right)\right.$ and $\left.\left(v_{H}, v_{H}\right)\right\},\left\{\left(v_{H}, v_{H}\right)\right\},\left\{\left(v_{L}, v_{H}\right)\right.$ and explicitly $\left.\left(v_{H}, v_{H}\right)\right\},\left\{\left(v_{L}, v_{L}\right),\left(v_{H}, v_{L}\right)\right.$ and $\left.\left(v_{H}, v_{H}\right)\right\}$. Large Loss: Subjects who mention either $\left\{\left(v_{L}, v_{H}\right)\right\},\left\{\left(v_{L}, v_{H}\right)\right.$ and implicitly $\left.\left(v_{H}, v_{H}\right)\right\},\left\{\left(v_{L}, v_{L}\right),\left(v_{H}, v_{L}\right)\right.$ and $\left.\left(v_{L}, v_{H}\right)\right\}$, $\left\{\left(v_{L}, v_{L}\right),\left(v_{L}, v_{H}\right)\right.$ and $\left.\left(v_{H}, v_{H}\right)\right\} . p=v_{L}$ : Subjects who mention outcomes: $\left\{\left(v_{L}, v_{L}\right)\right\},\left\{\left(v_{L}, v_{L}\right)\right.$ and $\left.\left(v_{H}, v_{L}\right)\right\}$. Mistake: Subjects who compute one of the payoffs wrongly.

In Table 26 we provide a detailed description of the classification of subjects based on their submitted prices in parts 1 and 2 as a function of the outcomes mentioned in the advice.

Table 27 reproduces the classification of subjects presented in Table 10 and adds the rows that show how many subjects observed at least one advice that mentions all four outcomes and how many observed no advice that mentions all four outcomes.

In the text we present the evolution of the classification of types in ADVPROB and ADVDET relative to the main treatments for subjects who are eventually classified as $V_{L} V_{H}$ (Figure 5), which is reproduced in the top row of Figure 8. In addition, Figure 8 also shows the evolution for subjects who are eventually classified as one of the other types.

\section{E Summary of Instructions}

Below we present a summary of how we describe the problem in the instructions that subjects are asked to read. Full instructions are available in the Instructions Appendix. Below, in upright letters we present lines that are specific to PROB. In italics and between brackets we show the corresponding line for DET. In bold we display lines that are common to both treatments.

- There is a company for sale. You can buy one company or none. The company's value is either A or B. [There are two companies for sale. You can buy two companies, one or none. 


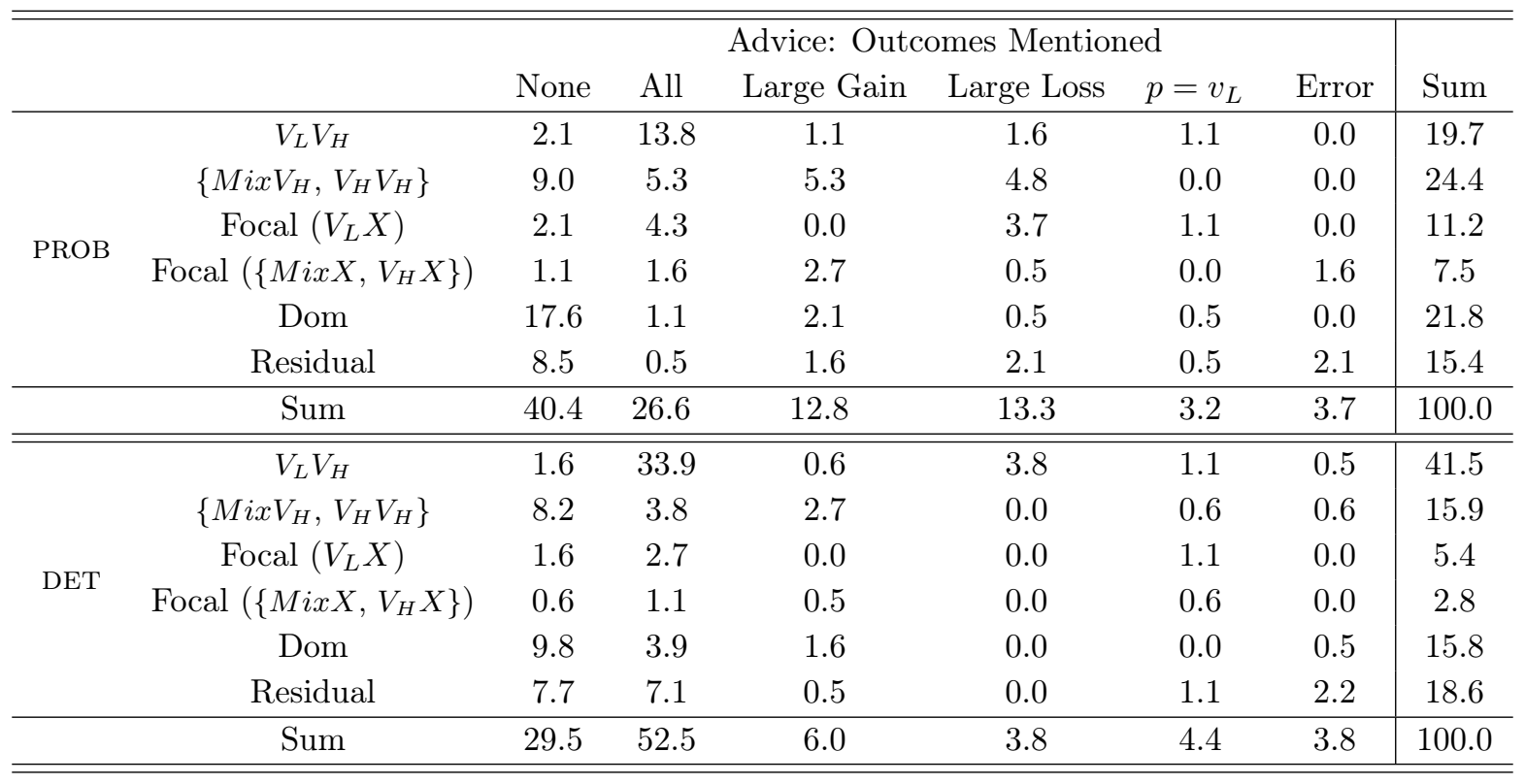

Table 26: Parts 1 and 2 (Type Classification) v. Part 3 (Advice Outcomes Categories)

Notes: 188 participants in РRов and 183 in DEт. There are four outcomes $(v, p)$ where $v, p \in\left\{v_{L}, v_{H}\right\}$. All (none) are subjects who mentioned all (none of the) four outcomes in any form. Large Gain: Subjects who mention either $\left\{\left(v_{L}, v_{L}\right)\right.$ and $\left.\left(v_{H}, v_{H}\right)\right\},\left\{\left(v_{H}, v_{H}\right)\right\},\left\{\left(v_{L}, v_{H}\right)\right.$ and explicitly $\left.\left(v_{H}, v_{H}\right)\right\},\left\{\left(v_{L}, v_{L}\right),\left(v_{H}, v_{L}\right)\right.$ and $\left.\left(v_{H}, v_{H}\right)\right\}$. Large Loss: Subjects who mention either $\left\{\left(v_{L}, v_{H}\right)\right\},\left\{\left(v_{L}, v_{H}\right)\right.$ and implicitly $\left.\left(v_{H}, v_{H}\right)\right\},\left\{\left(v_{L}, v_{L}\right),\left(v_{H}, v_{L}\right)\right.$ and $\left.\left(v_{L}, v_{H}\right)\right\}$, $\left\{\left(v_{L}, v_{L}\right),\left(v_{L}, v_{H}\right)\right.$ and $\left.\left(v_{H}, v_{H}\right)\right\} . p=v_{L}$ : Subjects who mention outcomes: $\left\{\left(v_{L}, v_{L}\right)\right\},\left\{\left(v_{L}, v_{L}\right)\right.$ and $\left.\left(v_{H}, v_{L}\right)\right\}$. Mistake: Subjects who compute one of the payoffs wrongly. $X \in\left\{V_{L}, M i x\right\}$.

\begin{tabular}{ccccccc|c}
\hline \hline & & $V_{L}, V_{H}$ & $\left\{\mathrm{Mix}, V_{H}\right\}, V_{H}$ & Focal & Dominated & Residual & Participants \\
\hline All & PROB & 24.4 & 22.0 & 22.0 & 14.6 & 17.1 & 41 \\
& DET & 72.5 & 5.0 & 10.0 & 7.5 & 5.0 & 40 \\
\hline Observed at least one Advice & PROB & 27.6 & 27.6 & 13.8 & 6.9 & 24.1 & 29 \\
that mentions all outcomes & DET & 71.8 & 5.1 & 10.3 & 7.7 & 5.1 & 39 \\
No Advice & PROB & 16.7 & 8.3 & 41.7 & 33.3 & 0.0 & 12 \\
mentions all outcomes & DET & 100.0 & 0.0 & 0.0 & 0.0 & 0.0 & 1 \\
\hline Selected Advice & PROB & 33.3 & 13.3 & 20.0 & 6.7 & 26.7 & 15 \\
that mentions all outcomes & DET & 78.1 & 3.1 & 6.3 & 6.3 & 6.2 & 32 \\
Did not Select Advice & PROB & 19.2 & 26.9 & 23.1 & 19.2 & 11.5 & 26 \\
that mentions all outcomes & DET & 50.0 & 12.5 & 25.0 & 12.5 & 0.0 & 8 \\
\hline
\end{tabular}

Table 27: Advisee Treatments: Parts 1 and 2 Type Classification [as \% of participants]

Notes: Type are defined based on the prices $p_{1}$ submitted in the last five rounds of part 1 and $p_{2}$ submitted in all five rounds of part 2. Type $V_{L} V_{H}: p_{1}=v_{L}$ and $p_{2}=v_{H}$. Type $\left\{M i x V_{H}, V_{H} V_{H}\right\}: p_{1} \in\left\{v_{L}, v_{H}\right\}$ and at least one $p_{1}=v_{H}$ and $p_{2}=v_{H}$. Type 'Focal': $p_{1}, p_{2} \in\left\{v_{L}, v_{H}\right\}$ and at least one $p_{2}=v_{L}$ (corresponds to $V_{L} V_{L}, V_{L} \operatorname{Mix}, \operatorname{Mix} V_{L}, \operatorname{MixMix}, V_{H} V_{L}$, or $V_{H}$ Mix. Type 'Dom': $p_{1}, p_{2} \notin\left\{v_{L}, v_{H}\right\}$. Residual: All remaining subjects. 


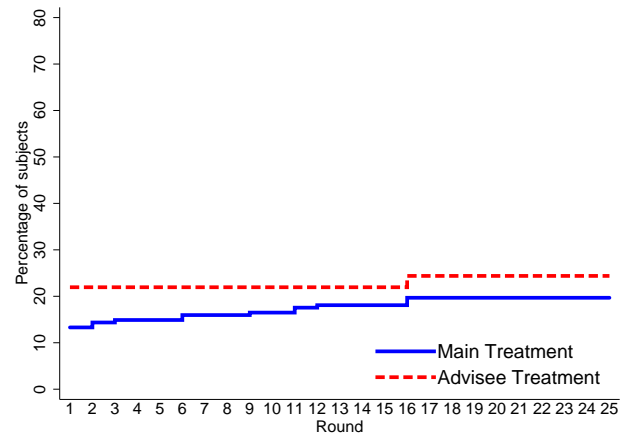

(a) Probabilistic: $p=v_{L}: n$ to $15 \& V_{L} V_{H}$.

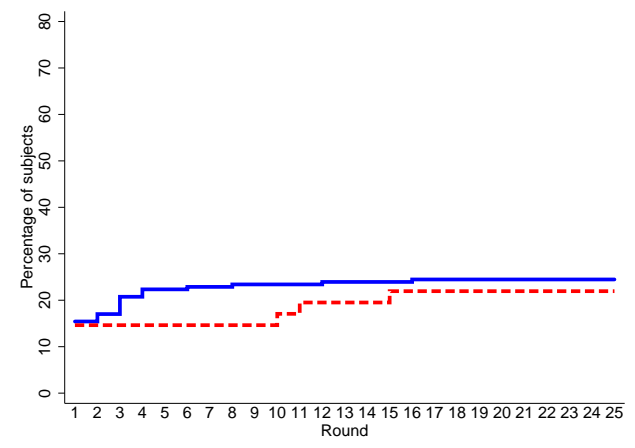

(c) Probabilistic: $p \in\left\{v_{L}, v_{H}\right\}: n$ to $15 \&$ $\left\{M i x V_{H}, V_{H} V_{H}\right\}$

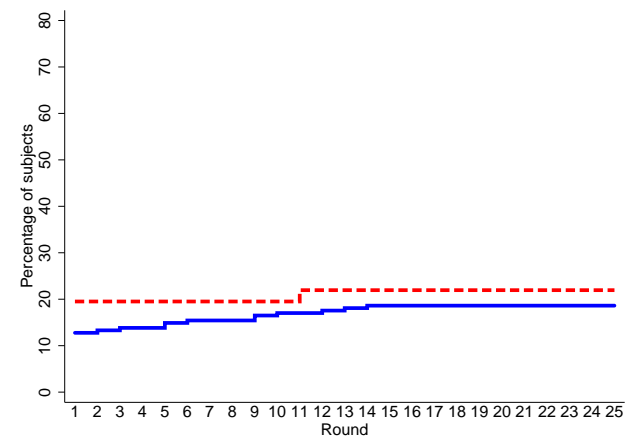

(e) Probabilistic: $p \in\left\{v_{L}, v_{H}\right\}: n$ to $15 \&$ Focal.

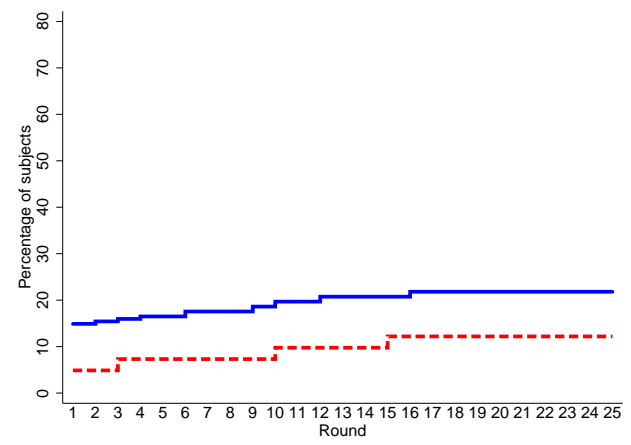

(g) Probabilistic: $p \notin\left\{v_{L}, v_{H}\right\}: n$ to $15 \&$ Dominated.

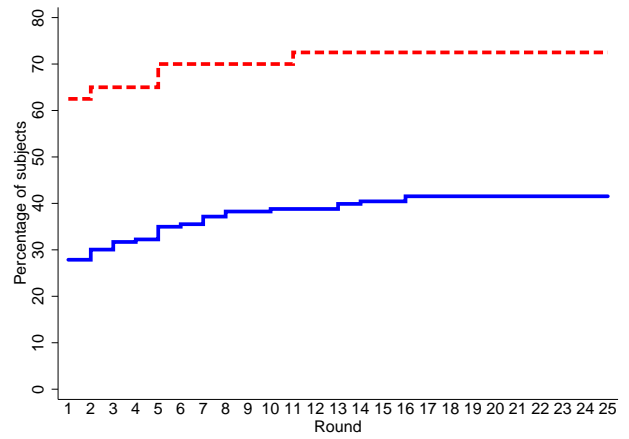

(b) Deterministic: $p=v_{L}: n$ to $15 \& V_{L} V_{H}$.

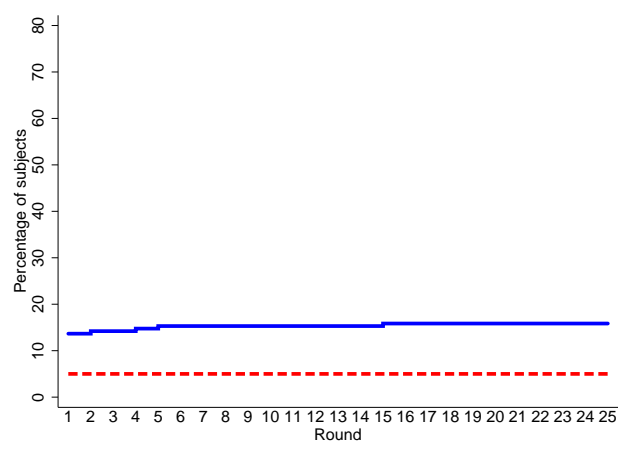

(d) Deterministic: $p \in\left\{v_{L}, v_{H}\right\}: n$ to $15 \&$ $\left\{M i x V_{H}, V_{H} V_{H}\right\}$

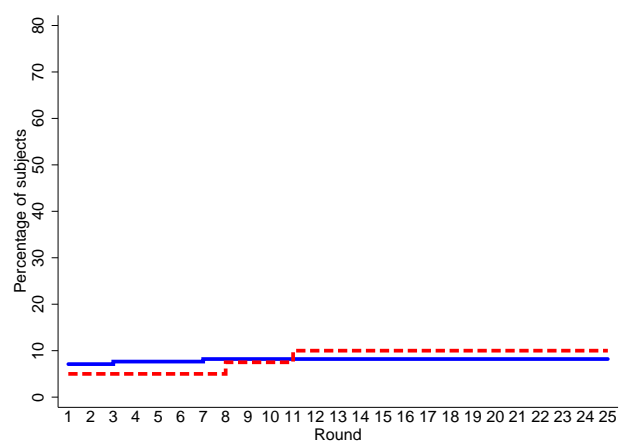

(f) Deterministic: $p \in\left\{v_{L}, v_{H}\right\}: n$ to $15 \&$ Focal.

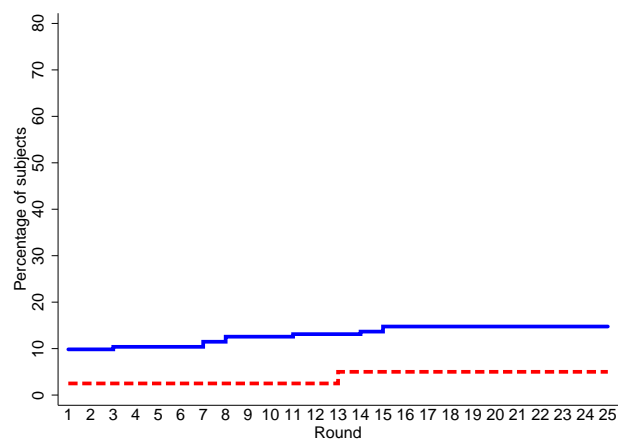

(h) Deterministic: $p \notin\left\{v_{L}, v_{H}\right\}: n$ to $15 \&$ Dominated.

Figure 8: Evolution of Types: Main Treatments and Advisee Treatments. 
The first company's value is A. The second company's value is B.] A and $\mathbf{B}$ represent two numbers. The numbers change from round to round.

- In each round you will learn the value A and B that the company may have. With equal chance the company's value is A or B. In each round, the interface is programmed to toss a coin and assign value $\mathrm{A}$ if heads comes up and value B if tails comes up. You will not know which of the two values is selected. [In each round, you will learn the value $A$ of the first company and the value $B$ of the second company.]

- You will submit one price. You can submit any price from 0 to 150 . This is the price that you are willing to pay for the company [a company].

- You do not know if the company for sale is of value A or of value B. [You do know that the two companies for sale are the first company of value A and the second company of value B.]

- Transaction for the $[e a c h]$ company:

- If the price you submit is higher than or equal to the value of the company, you buy the company.

- If you buy, you increase the company's value by $50 \%$. This means that if you buy the company of value A [the first company of value $A$ ], the value to you is $1.5 \times A$. If you buy the company of value $\mathrm{B}$ [the second company of value $B$ ], the value to you is $1.5 \times B$.

- If you buy the company, your profit is: 2 times (1.5 x value of the company - price). [For each company you buy, your profit is: $1.5 x$ value of the company - price.]

- [For each company, If the price you submit is lower than the value of the company, you don't buy the company and your profit is zero.

- [The price you submit is the same for both transactions. You can make money from both transactions.]

- Your payoff for the round is the profit from the transaction. [Your payoff for the round is the sum of the profit from the two transactions, the transaction of the first company and the transaction of the second company.]

For screenshots of the round 1 part 1 problem with $v_{L}=20$ and $v_{H}=120$ in PROB and DET, see Figure 9 . 


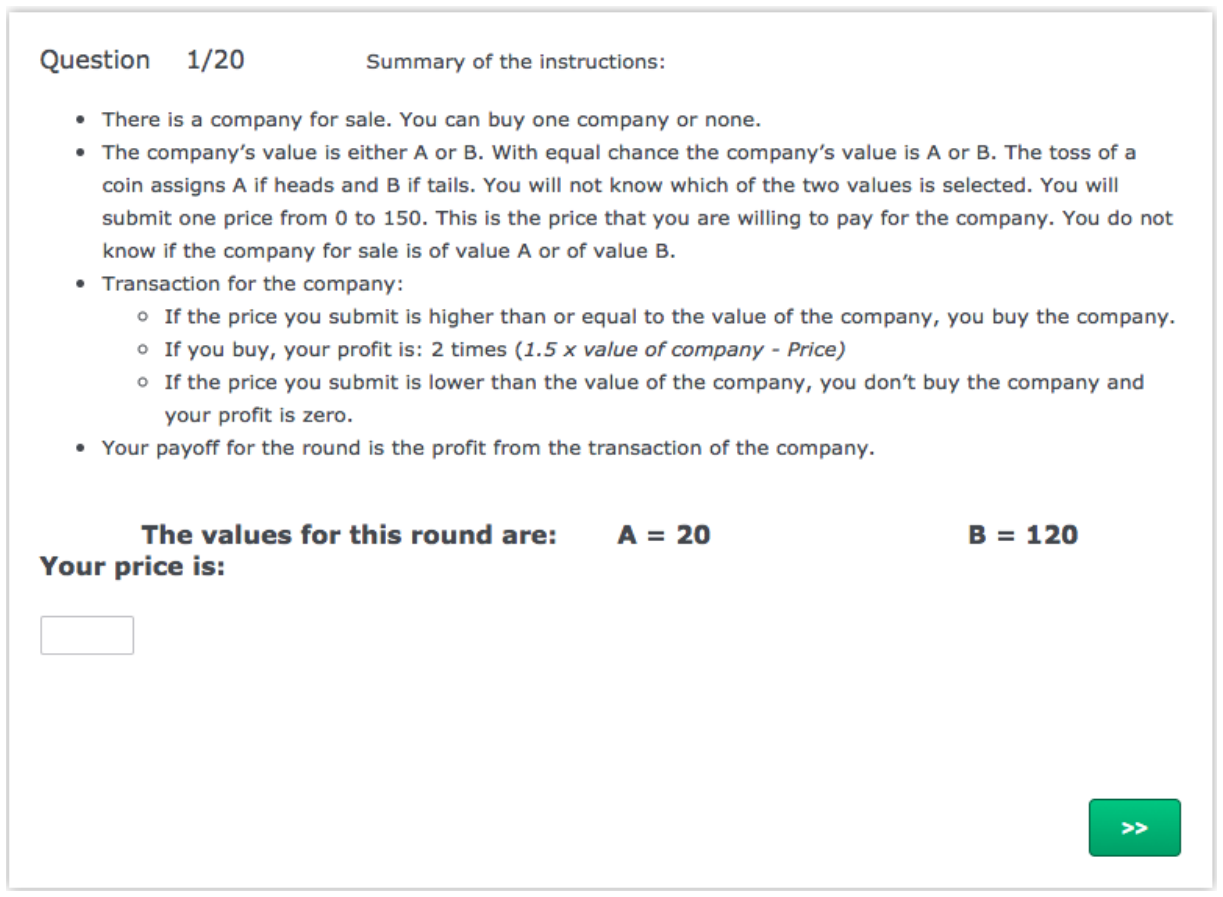

(a) PROB

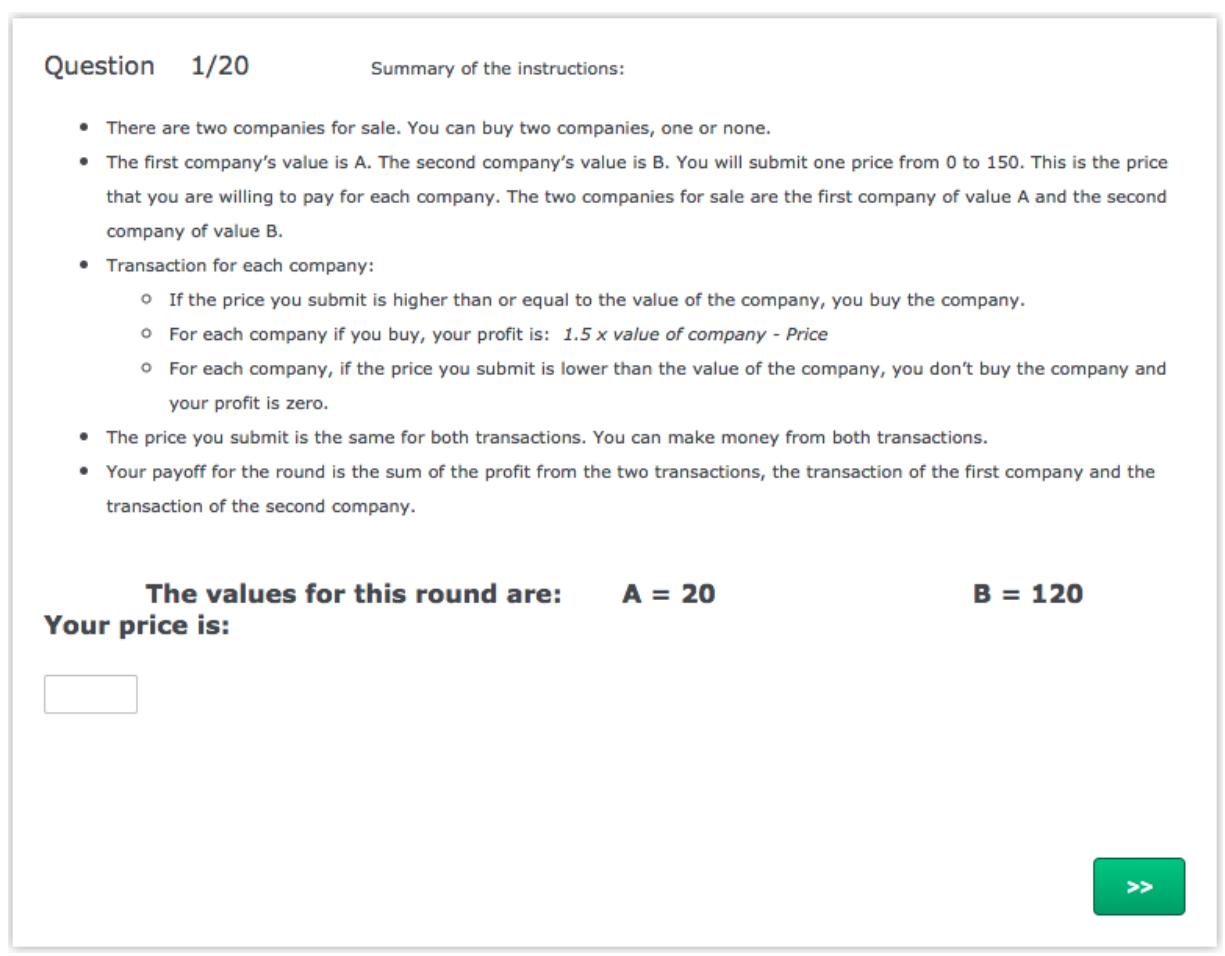

(b) DET

Figure 9: Screenshots of Part 1 Round 1 


\section{F Examples of Advice}

After the examples, we provide comments as to why the advice was classified in the corresponding category, in cases where it is not obvious. The advices mentioned below are verbatim, and hence they may contain mistakes or grammatical errors. To make recommendations, subjects use the terminology used in the instructions. For example, they refer to a company of low value as company A and to a company of high value as company B.

\section{PROB}

- Computes both payoffs explicitly: "You should not go with an offer in the middle of the two values, as going above 20 reduces your profits if $A$ is selected but also does not help your chances of purchasing $B$ if that is selected. So the two options are bid 20, if $A$ is selected you make a profit of 20, if $B$ is selected you make 0, making an average value of 10. If you bid 120, if $A$ is selected your profit is -180, is B is selected your profit is 120, making an average value of -30. Statistically based on the law of averages you should always bid the value of the lower option".

- Payoffs mentioned qualitatively: "I would submit 20. Worst case you would sit at 0 profit. Best case $A$ is selected a you would make profit. I don't think submitting 120 is worth the risk." Comments: Outcome $(v, p)=\left(v_{L}, v_{H}\right)$ mentioned (Worst case you would sit at 0 profit); Outcome $(v, p)=\left(v_{L}, v_{L}\right)$ qualitatively mentioned (Best case $A$ is selected a you would make profit). Outcomes $\left(v_{H}, v_{L}\right)$ and $\left(v_{H}, v_{H}\right)$ qualitatively mentioned when the subject states: ' $I$ don't think submitting 120 is worth the risk.' We code this recommendation as mentioning the outcomes for the following reason. The phrase 'worth the risk' indicates that there is a potential gain, but that the risk is too high for it to be worth it.

- Mentions outcomes, but does not compute Expected Payoffs: "I would advise you to bid 20 for the company. This would decrease your risk to "0" but your possible profit to "20". If you bid 120 for the company, you could profit 120 but if the company selected is " $A$ " then your would lose 180. There is no risk with bidding 20 since if " $B$ " is selected your profit is " 0 ". So bidding 20 is the selection with no chance of losing money."

- Advice mentions large losses "You should enter the price that is equal to value A, the lower value. If you pick a higher price to get the higher value, if the lower value is selected instead you could lose money."

- Advice mentions large gains "I think you should always go with the possible higher amount of $B$ since it will result in more of a profit for you if it happens to be the selected company."

- Advice mentions $p=v_{L}$ outcomes: "I would be willing to pay \$20. In my opinion that is the safest amount. If you are lucky and the value is $A$, you make money, if the value is $B$, you loose nothing." 
- Mentions no outcome: "70, provides a good amount."

- Makes some mistake: "You will make a lot more money if B is chosen but there is still a chance $A$ will be chosen. If you choose 20 you will definitely make some money. But if you choose a number higher than 20 is $A$ is chosen then you cannot buy the property and your profit is zero. If you are a risk taker, choose a value higher to or equal to 120. I hope that helps you." Comment: We do not code an advice as including a mistake if the subject is conceptually correct, but he/she makes a mistake in arithmetic. We only count as mistakes when there is some conceptual mistake in the advice. In the case of the example recall that if $p \geq v_{L}$, then a company of value $v=v_{L}$ is purchased. The following sentence is conceptually incorrect: 'But if you choose a number higher than 20 is $A$ is chosen then you cannot buy the property and your profit is zero.'

DET

- Computes both payoffs explicitly: "My only advice is to do the math for each company purchase. To purchase both companies you are looking at now will cost 120 for EACH at a minimum. While the value of company B will be 180 to you, the final value after your purchase cost will be 60. Seems like an OK prospect until you factor in the loss you will take on the purchase of company A. You will spend 120 to purchase to company A which only has a value of 30 to you when you purchase it. Subtract the purchase cost of 120 and you are left with -90. Add-90 (loss from the purchase of company $A$ ) and 60 (profit from the purchase of company B) and you are left with a total loss of -30 for the transaction overall. I would submit no more than 20 for an offer and only purchase company A which would represent a profit of 10."

- Computes one payoff explicitly: "To buy you offer 120 for each. Making the profit of A: 30 $120=-90$. And The profit of B: 180-120=60. The combined profit of $A:-90+B: 60=-30$. Creating a loss of -30 on the total deal. It is better to only purchase $A$. The 120 cost of $A$ when purchasing both creates a loss." Comment: Outcomes corresponding to $v=v_{H}$ are explicitly mentioned and computed. Consider the phrase: 'It is better to only purchase A.' With this phrase the subject implicitly mentions that there is no gain if the company is that of high value ('only purchase A'). At the same time, by mentioning that it is better to purchase A the subject is implicitly recognizing that there is a positive payoff from buying just the low value company (A). We code this as the subject qualitatively (or implicitly) mentioning outcomes related to $v=v_{L}$.

- Payoffs mentioned qualitatively: "Only Bid 20. This way you will purchase company A and make a small profit in the end. When there is a large difference between the values of company $A$ and company $B$, only buy company $A$. If you spend 120 and buy company $B$ as well, then the amount you will lose from the purchase from company A having spent 120 for it, will offset any profits that you make from the purchase of company $B$, putting you in a negative profit which 
means you lose money." Comment: Outcomes related to $p=v_{L}$ are implicitly mentioned in this sentence: 'This way you will purchase company $A$ and make a small profit in the end.' The subject implicitly recognizes that bidding 20 will not buy both companies and qualitatively mentions that there is a profit from buying the low value company. Outcomes related to $p=v_{H}$ are also qualitatively mentioned in the last sentence of the advice.

- Advice mentions large gains: "Buy company B profit will be 180."

- Advice mentions large losses: "Only buy A, you will lose money if buy both."

- Advice mentions $p=v_{L}$ outcomes: "buy the first one at 20 for a profit of 10."

- Mentions no outcome: "Do the math for your equations to ensure to get a good result."

- Makes some mistake: "I would submit a price of 120. With this price, there is a direct profit since the value will increase by 50\%. If you own both, the sum of $140 \times 1.5-120$ would result in a net profit of 90 and makes this a profitable purchase. Even if you only purchase $B$ you would still make a profit of $60(120 \times 1.5$ - 120 = 60). " Comment: We do not code an advice as including a mistake if the subject is conceptually correct, but he/she makes a mistake in arithmetic. One mistake in this advice is in the last sentence. It is not possible to offer a price that only purchases the high-value company. 